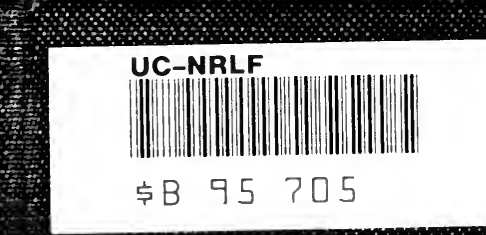




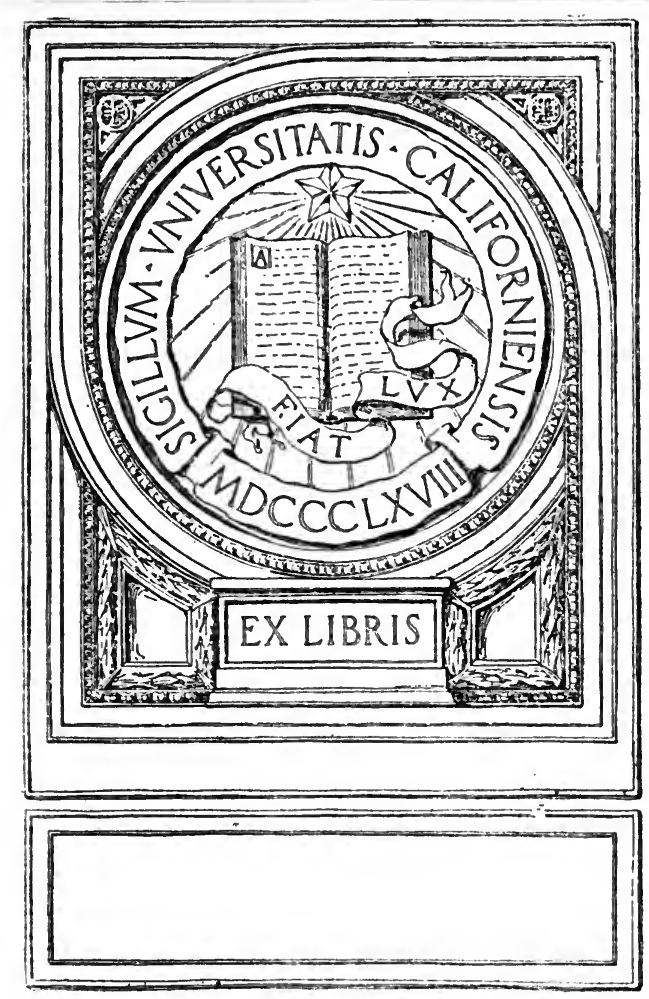



Digitized by the Internet Archive in 2007 with funding from Microsoft Corporation 
PUBLICATIONS OF THE UNIVERSITY OF MANCHESTER

ECONOMIC SERIES

No. VI

DYEING IN GERMANY AND AMERICA 
Published by the University of Manchester at THE UNIVERSITY PRESS (H. M. McKechnie, Secretary)

Twelve Lime Grove, Oxford Road, MANCHESTER

LONGMANS, GREEN \& CO.

LoNDoN : 39 Paternoster Row

New York : 443-449 Fourth Avenue and Thirtieth Street

BombaY : 8 Hornby Road

Calcutra : 303 Bowbazar Street

MADras : 167 Mount Road 


\section{DYEING IN GERMANY}

\section{AND AMERICA}

\section{WITH NOTES ON COLOUR PRODUCTION}

BY

S. H. HIGGINS, M.Sc.

LATE GARTSIDE SCHOLAR OF THE UNIVERSITY AND DEMONSTRATOR IN DYEING, MANCHESTER SCHOOL OF TECHNOLOGY

DYERS COMPANY GOLD MEDALLIST FOR RESEARCH, 1913

TECHNICAL CHEMIST AND WORKS MANAGER

SECOND EDITION, RE-WRITTEN AND ENLARGED

MANCHESTER :

AT THE UNIVERSITY PRESS

Twelve Lime Grove, Oxford Road

LONGMANS, GREEN छ CO.

London, New York, Bombay, etc. 


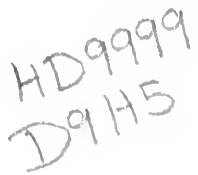

PUBLICATIONS OF THE UNIVERSITY OF MANCHESTER

No. CVIII

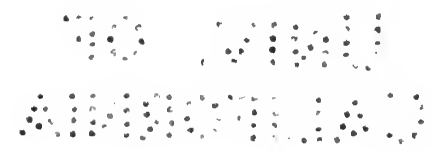




\section{PREFACE TO THE SECOND EDITION}

THE first edition of this book was issued in 1907 as " a Report to the Electors of the Gartside Scholarship of the University of Manchester on the Results of a Tour in the United States of America and Germany in 1905-1906," and was intended to give a general survey of the dyeing and allied industries of these countries.

The very favourable reception awarded to the book has exhausted the supply of copies and necessitated the issue of this new edition. Although no further visit has been paid to either of the countries, I have been able to add considerably to what appeared in the first edition, chiefly as the result of experience gained during the past nine years, firstly as a demonstrator in the Dyehouse of the Manchester School of Technology, and secondly, as a works chemist and manager. Naturally, this experience is most in evidence in those parts of the book dealing with the branches of the industry with which $I$ am the most familiar. I have also taken the liberty of including certain material which I have published in various journals since the issue of the first edition.

It is hoped that this edition will be useful to the student requiring a general knowledge of the industry, to the publicist seeking a comparison between the industrial conditions in this and other countries, to the general reader, and also to the practical dyer, who may find in these pages some things of value and interest to him.

In the preparation of this edition I have received much assistance from the many helpful suggestions made by reviewers when the book was first published. I am also indebted to these gentlemen for the rectification of several errors which appeared therein. I wish to place on record my appreciation of the care taken in the publication of the present edition by H. M. McKechnie, Esq., Secretary of the Manchester University Press; also to repeat my thanks to Arthur Jones, Esq., M.A., for the help he gave me with the first edition.

S. H. HIGGINS.

Luncarty, Perth, N.B.,

July 1916. 



\section{CONTENTS}

Preface .

\section{CHAPTER I}

General .

The art of dyeing - Influence of chemistry-Opposition to progressHank-dyeing and washing-Cloth-dyeing.

\section{CHAPTER II}

Cop-DYeing

Its object-Selection of colours-The difficulties involved-Machines of two classes-Comparison of these machines-Three important machinesPerfection not attained.

\section{CHAPTER III}

\section{SUlphor Colours and Indigo}

Fastness of sulphur colours-Importance as cotton dyes-Their tendering action on cotton-Use for wool dyeing-Machines used for dyeing sulphur colours-Natural and synthetic indigo-Substitutes for indigoSulphur blues and indigo.

\section{CHAPTER IV}

\section{Mercerising}

Invention of the process-Hank-mercerising machines-Piece mercerising-Cooling of the lye-Recovery of the caustic soda-Recent scientific work on mercerising.

\section{CHAPTER V}

\section{Bleaching}

Development of the industry-Effect of chemistry - Efficient scouringContinuous scouring - Open-width scouring - Electrolytic bleachingSteeping of goods-The waxes of vegetable fibres-Bleaching without boiling.

\section{CHAPTER VI}

\section{Grrman and English Fuannelettes}

Use of raising-Cotton blankets-German production-Large-scale production-German and English methods compared-External and internal specialisation-Inflammability of flannelettes. 


\section{CHAPTER VII}

The Industry in the United States . . .

American conditions-Scarcity of labour-Large-scale productionUncertainty of their industry-Lack of specialisation-Effect of the tariff.

\section{CHAPTER VIII}

Lowell, Lawrence, and Fall River, Mass.

Lowell-The town and its industry-Merrimack Manufacturing Company-Hours and Conditions-Turning to finer work-Production of machines-Employer and employed-Hamilton Manufacturing CompanyArrangement of works - American worker - Lawrence and its industryPemberton Works-Pacific and Arlington Mills-Fall River-Water supply-Fall River Bleachery-The drying of cloth-Barnaby WorksBridgeport, Conn. - Salts Manufacturing Company - Klauder-Weldon dyeing machines.

\section{CHAPTER IX}

\section{Conditions of LIFE IN THE Industry . . .}

Injurious conditions in dyeing-England, Germany, and America compared-The power of the workmen in Germany-The development in America-Wages in the three countries-America's high wages-Short hours-The German workman and the American.

\section{CHAPTER $\mathrm{X}$}

EFFiciency in the Industry

Dyeing in Germany-Application of science to the art-The position in America-English workmen in America-Empiricism and scienceArrangement of the works-Using up of wastes-Aids to manufactureSpecialisation in the industry-Dye-works and machine shops-High specialisation in England-British pessimism.

\section{CHAPTER XI}

Instruction IN DYeING • • • • •

The dyeing industry subject to change-British pessimism regarding education-Appointments for technical students-German technical schools -Education of the German workman-American schools-Suggested reforms.

\section{CHAPTER XII}

\section{Colodr Production}

The German works-Past, present, and future-Combination and its possible effects-Our position - Possible remedies-Protection-Patent Legislation-Organisation of the industry in Germany.

\section{CHAPTER XIII}




\section{CHAPTER I}

\section{GENERAL}

The art of dyeing-Influence of chemistry-Opposition to progress-Hankdyeing and washing-Cloth-dyeing.

The art of dyeing is very old, as the following quotation from Pliny shows: "Garments are painted in Egypt in a wonderful manner, the white cloths being first smeared, not with colours, but with drugs which absorb colours. These applications do not appear on the cloths, but when the cloths are immersed in a cauldron of hot dyeing liquor they are taken out painted the moment afterwards. It is wonderful that, although the dyeing liquor is only of one colour, the garment is dyed by it of several colours, according to the different properties of the drugs which have been applied to the different parts : nor can this dye be washed out. Thus the vat, which would doubtless have confused all the colours if the cloth had been immersed in a painted state, produces a diversity of colours out of one, and at the same time fixes them immovably." Thus we have evidence that at an early period the practice of what is now known as polygenetic colour-dyeing was carried out. Since such knowledge of dyeing existed in these early times it is surprising that it is only during the last few centuries that marked progress has been made. The discovery of America influenced the art of dyeing so materially that its real development dates from that event. Mexico furnished cochineal, and, later, the beautiful tropical indigo displaced to a large extent the ancient woad of England. We find that the law restricted the dyeing industry in all countries : sometimes the methods of dyeing were strictly defined and no others were permitted; at other times the districts in which dyeing was 
allowed were stated; and in some periods a duty was placed on all dyed cloth. For instance, at one time logwood was not allowed to be used for dyeing blues, as the colours thus produced were said to be fugitive. An Act of Parliament of $\mathbf{1 5 5 2}$ limited the number of colours which might be used; William III. added to the list; and later legislation allowed the free use of dye-stuffs. These obstacles hindered, to some extent, the progress of the dyeing industry, but it is only in quite recent times that great advances have been made.

The introduction of chemistry into the industry has indefinitely enlarged its resources by showing how to produce varieties of colours extending through the whole range of the prismatic spectrum, and has enabled the dyer to fix and render durable many of those beautiful vegetable dyes the ephemeral nature of whose brightness formerly detracted from their utility. Along with these improvements the nature of the substance to be dyed has received careful scientific consideration; the processes have been adapted to suit the fibres being dyed, and mordants have been introduced by means of which peculiar shades have been produced, the most diverse fabrics dyed together and perfect fixity given to the colours.

Yet, during its rapid development, the dyeing industry had to combat many prejudices, the greatest of which was the reluctance of the dyers themselves to adopt the new methods when introduced. The utility of colours dyed without mordants was not at first recognised, and it was a long time before the substantive cotton colours could get a footing. The general adoption of metallic mordants was opposed; but the discovery of alizarine red and, later, of alizarine blue brought in this method, and it has gradually grown in use until now it is one of the most important methods of dyeing. It must be remembered, however, that every improvement in every trade is received with opposition of some kind, and the history of dyeing shows the industry to have been no exception to the rule. The preliminary difficulties may be great, and unwillingness to discard the old methods may exist, but the fact that the new methods gain acceptance in other quarters show their utility, and ought to urge all beyond initial obstacles. 
As to the location of the dyeing industry, we have in Germany :

1. North-West Germany, including Rhineland and Westphalia. In this district the principal centres are Crefeld for silk, München-Gladbach for cotton, and ElberfeldBarmen for general manufacture, especially dyeing.

2. Middle Germany, containing what is usually called "the Saxony Industry" and spread over a large area. The industry in this district is very old. Chemnitz and Zwickau are important centres.

3. South-West Germany, where the industry is connected with the French and Swiss industries. Mülhausen is the principal town. Mülhausen is an important fine cotton centre, and much fine printing is done in this district for English and French firms. The work is totally different from that of other parts of Germany. The MünchenGladbach industry is largely devoted to the production of cotton blankets. In Chemnitz the dyeing is of a varied nature. The machines used in the textile districts mentioned are in many cases of British manufacture, or copies of the same, and even in the trade schools British machinery is used. Yet the Germans are now producing a large number of textile and dyeing machines, and even exporting them to Britain.

In America the dyeing industry is scattered. In the Philadelphia district the dyeing of carpets and coarse goods is the principal business. In the Southern States, where bleaching and dyeing are comparatively new industries, there are some fine works ; but the works of the New England States are the most important for the present review. Nearly everything that is manufactured anywhere in the United States is made in New York State. It has a population of over 7,000,000, a foreign commerce only exceeded by four countries, and is the leading State of the United States in many important industries. Yet, turning farther north, we find New England contains twothirds of all the cotton spindles and three-quarters of all the looms of the United States, while Fall River makes three-quarters of the print cloth produced in that country.

Dyeing in Europe was first effected in the yarn form, and this method is still largely used. Hank-dyeing has 
not changed much of recent times, and in dye-houses in all countries men can be seen standing over wooden vats changing periodically the wooden sticks, from which the hanks hang into the dye liquor. The hanks are lifted, turned, and dropped back again, while to improve the evenness of the dyeing the positions of the sticks are changed regularly so that all experience the same concentration of dye-stuff.

Machines have been devised to imitate this movement, but few of these have been adopted to any large extent. Such machines are really only suitable for dyeing large quantities of yarn the same colour. The author did not see any such machines at work in Germany, but in America the Klauder-Weldon machine for this purpose was often seen (see p. 63). In the United States, however, hankdyeing is not so important as it used to be. They make so many cloths where the warp is the same count as the weft, that both warp and weft are receiving the same treatment before weaving. Both are dyed together in the warp-dyeing machine, and then taken, part to be quilled and part to be beamed. This is said to be a cheaper process than separately treating the warp and weft yarns in the more usual way. . As regards hank-dyeing machines, it is evident that they can never wholly replace hankdyeing by hand, for certain colours require special care, and it is useless to attempt to design machines to meet each special operation.

After dyeing, the hanks must be washed, this operation being of importance. Machines have been devised for this purpose. The simplest method is to pass the hank alternately through water and between a pair of squeezing rollers. The washing-machines are again designed to imitate washing by hand. Thus, in one machine the hank is lifted out of the water, plunged rapidly in again, and at the same time turned.

Haubold's-a large machine firm in Chemnitz, Saxony -build a machine which is very economical and has a large production. It consists of a circular vat with a partition fixed radially at one part of the rim of the vat. Fresh water is conducted in at one side of this partition and the dirty water flows away at the other side, that is, after 
having traversed round the vat and washed the hanks which are suspended from copper rods placed radially on the vat. Each of these rods is covered with a square porcelain part on which the hanks hang. The porcelain parts revolve, thus moving the hanks in the water, while the whole wheel carrying the hanks moves round with a jerky motion. During the revolution of the wheel, the hanks first meet dirty water then cleaner water as they revolve, until finally they meet clean water and are taken out. The hanks are well and rapidly washed, and as the washing is on the counter-current system, the water is economically used. The machine is supplied in three sizes, with 12,18 , and 27 copper radii, and is suitable for all classes of yarn.

Hank-dyeing and washing are still principally done by hand, and at Chemnitz workmen are seen standing on low bridges crossing the river and washing hanks in the running water. This peculiar sight may be witnessed all along the winding river. In the Barmen district such a practice is prevented by the bad state of the river, for only on a very wet Sunday, and when the factories have been closed some hours, can the Wupper be considered worthy of such usage.

Cloth-dyeing is done on the jigger or on the rope form, and big works abroad were seen using both methods. The dyeing of mordanted cloth was done in the rope-dyeing machines, as the mordanting assured even dyeing and made the use of the jigger unnecessary. It was a frequent occurrence to see a line of rope-dyeing machines at work in German works. These machines require little attention, but because of the difficulty of washing it is almost necessary to have different machines for different colours before good results can be obtained. This remark also applies to the use of jiggers for dyeing. In Germany some of the jiggers had extra rollers so as to increase the time of immersion of the cloth in the dye liquor; but since dyeing is really done more in the batch than while in the liquor, the advantage of these rollers is questionable.

At Mülhausen, as at Chemnitz, the river is used directly for washing, and washing-machines are directly built over branches of it. Most of the works congregate about the rivers and streams. 


\section{CHAPTER II}

\section{COP-DYEING}

Its object-Selection of colours-The difficulties involved-Machines of two classes-Comparison of these machines-Three important machinesPerfection not attained.

UNTIL comparatively recently, cotton yarn had to be dyed in the hank form: the cop produced by the spinningmachine was unwound, the yarn made into the hank form, dyed, then rewound to the cop form and placed in the shuttle. During this operation of unwinding and rewinding, cotton was lost owing to the entangling of the threads, and cop-dyeing arose to save the trouble mentioned and thus to economise both labour and material. Cop-dyeing originated in the Manchester district about thirty years ago, and has now assumed considerable dimensions in Germany. Many machines are in use for cop-dyeing, but the success of the operation seems to depend not so much on the machinery used as on the proper selection of the dye-stuffs and on the careful handling of the machines.

During the dyeing operation the dye liquor has to be forced through the cop, and in this way the material of the cop acts as a kind of filter for the dye-stuff. It is therefore evident that success in cop-dyeing can best be attained by using perfectly soluble colours, which leave no residue in the dye-bath; otherwise there is a tendency towards uneven dyeing, and to concentration of the dyestuff on the outside of the cop. This is partly counteracted in some machines by periodically reversing the direction of flow of the dye liquor through the cop.

Consideration shows us that many colours, as aniline black, turkey red, etc., cannot be dyed with success on 
cops ; others, such as indigo, are difficult to manage, The dyeing with sulphur colours is also difficult because of their tendency to oxidise on the outside of the cops, thus producing unevenness. In using these colours, the cops must be kept immersed in the dye liquor during the dyeing operation. Those colours which are slowly taken up by the fibres are the best to use in cop-dyeing, as even penetration is obtained; dyeing with basic colours, which act quickly, is difficult. To overcome the difficulties mentioned, various precautions are taken, and the colour firms give every assistance in that direction by their carefully prepared recipes. Moreover, in cop-dyeing the difficulties are accentuated by the fact that any mistakes are not revealed to the dyer, who cannot tell if he is sending black cops with white cores to the weaver. If the latter is not an observant person, pieces of cloth may thus be spoiled.

The machines for cop-dyeing are of two classes: those in which the cops have solid spindles, and (2) those in which the spindles of the cops are perforated.

In the first class the cops containing the solid spindles, which serve to keep the cops from becoming damaged, are packed together into as uniform a mass as possible by means of cotton waste and other such material. Steam is first sent through the mass and then the dye liquor. The success of the operation depends on good packing, for, if the dye liquor passes along certain channels only, the dyeing of the cops is not uniform. One of the oldest of these machines and the simplest is the Mommer machine, in which the cops are tightly packed in a square box which has two of its opposite sides perforated. The dye liquor is pumped between these perforations and through the mass in the box. By reversing the direction of the dye liquor more even penetration is obtained, for, if certain channels are produced during the passage of the dye liquor in one direction, it is not likely that channels will be formed in the same places when the pump is reversed and the dye liquor travels in the other direction. In Cohnen's machine the cops can be hydro-extracted immediately after dyeing, by merely revolving the box in which the cops are packed. The machine acts in the same way as the one previously described, but the arrangement 
for hydro-extracting is very convenient. The cops can thus be dyed, washed, and hydro-extracted without taking out of the machine. The machine is specially suitable for dyeing basic colours on cops, for in this case it is necessary to hydro-extract after each operation if uneven dyeing is to be avoided. The Obermaier machine, such as is used for dyeing loose fibres, can also be used for dyeing cops with solid spindles.

Interest in the solid spindle machines, however, is rapidly decreasing, and attention is being given to machines of the second class. Some machines can, however, be adapted to dye by both systems. For instance, the author saw a machine made by Schirp of Barmen being used for dyeing cops with solid spindles, whereas this machine is usually employed for dyeing according to the other system.

The penetration of the dye liquor in the machines using perforated spindles is more uniform, and this factor has decided in their favour. Further, the power to drive the dye liquor through the cops is much less than in the first system. In the second system, the dye liquor is forced into the perforated spindle on which each cop is placed, through the perforations of the spindle, through the cop surrounding the spindle, and back into the dyebath. In this way each cop receives individual treatment, instead of being penetrated by the dye liquor in a mass as in the first system. Formerly there was a difficulty in transferring the cops to and from the metallic spindles used in these machines, but, later, perforated paper spindles were introduced, which remain inside the cops during the operations and keep the threads in position. The metallic spindles of the dyeing machines fit inside the paper spindles. After dyeing, the cop along with the paper spindle is taken to the hydro-extractor.

One case of uneven cop-dyeing, which had been caused by the paper spindle, came to the author's notice while in Germany. The inside of the cop, immediately next to where the paper spindle had been, was more deeply dyed than the remainder of the cop. Investigation showed that the alum used in the manufacture of the paper had acted as acid to the dye liquor, drawn the dye-stuff from solution, 
and concentrated it on the part of the cop immediately next to the paper.

In the case of the perforated spindle machines the direction of motion of the dye liquor is altered from time to time by reversing the pump, in order again to ensure uniformity of dyeing. Any impurities or solid particles of any kind in the dye-bath, which rise to the top of the dye-bath, tend to fill up the top holes of the perforated spindles and to thus produce uneven dyeing.

Modern perforated spindle machines : (1) Grämiger's, (2) Haubold's, (3) Schirp's, and (4) Pornitz's. Grämiger's, one of the first of this type, has been improved in England, and is used for dyeing indigo on cops. Haubold's machine was seen at work by the author in the dye-works of Herr Haase, its inventor. This works is situated at Neukirchen, a few miles over the hills from Chemnitz, and is principally concerned with the dyeing of cops and stockings. Chemnitz has a large business in the dyeing of stockings. The cop-dyeing machine consists of two parts, a dye-bath of tun-dish shape and a receptacle for the dye liquor. The bottom of the dye-bath is perforated, and in these perforations the spindles carrying the cops are screwed. The dye-bath is connected through a pump with the dye-vessel. The pump draws the liquor from the dye-vessel, forces it through the cops into the dye-bath until the liquor fills the dye-bath, and then draws some of the liquor back again through the cops into the dye-vessel. The cops are kept wholly immersed in the dye liquor during the process, and many alternations of the pump ensure even dyeing. The passages of the dye liquor, first in one direction and then in the other, take place about twenty times during an hour, according to the nature of the dyeing. If four machines are run together, as was the case at this works, one engine suffices for all, but with single machines the cost of driving becomes relatively higher. The machine, which was first built in 1892 , costs about $£ 600$. It is suitable for cotton and wool cops, and the products, the author found, stood minute examination. After dyeing, the cops were put in small baskets and arranged in a circle inside a hydro-extractor. After hydro-extracting they were dried in a chamber through which a strong 
current of hot air was passed. If the cops dry too slowly, there is a tendency for the upper side of the cop to dry first, to extract the dye liquor from the lower part, and thus produce uneven dyeing.

The Schirp machine consists of a stout square box with removable sides. In the middle of this box is another box, which might be considered a longitudinal section of the larger one. On both faces of the smaller box the perforated spindles carrying the cops are placed, so that the spindles project into the space of the larger box. The smaller box is fitted with the cops, wheeled into position, and then the dye liquor is forced into the small box, through the cops into the larger box, and round again in circulation. As in the other cases, the direction of the dye liquor is reversed from time to time.

In the Pornitz machine the cop carrier is a perforated cylinder, the cops taking up the whole of the round surface. When the cops have been fitted into position, this cylinder is lowered by means of a chain into the dye-bath, which is a cylindrical vessel big enough to admit the cops. By means of compression and suction the dye liquor is made to pass from the dye-bath through the cops into the dye reservoir. Fresh dye-stuff can be added to the liquor while in the reservoir, and in this way slow and even dyeing assured. The suction and compression are performed by means of a double-acting air-pump. The machine can also be used for dyeing warps; for this purpose the warps are wrapped round the cylinder of the machine over the perforations, which usually carry the cop spindles. The dye reservoir is usually placed underneath the dye-bath so as to save space.

In all these machines the cops have first to be steamed and hot water sent through them before dyeing, in order to ensure even penetration. Penetration is of course assisted by " boiling out " the cops on a kier before dyeing, or by adding turkey red oil to the water used for " wetting out" the cops.

At one works cops were seen being dyed in a foam dyeing-machine. The cops were tightly packed in the top of an upright cylinder, while at the bottom of the cylinder the dye liquor, containing soap, was made to 
froth up by means of steam. When the bubbles burst, the air round the fibres is driven away and the dye-stuff attracted. This method is said to be successful in dyeing certain shades on cheeses.

The author saw no cop-dyeing machines in the United States ; cop-dyeing is too delicate a process for the American class of trade.

Nearly all the cop-dyeing machines in use in Germany are very similar to each other, different forms being merely modifications of the same principal idea. The production of machines for sale is as yet little developed. Mommer of Barmen informed the author that they no longer build their machine, because there is no demand for it.

Of recent years the dyeing of cops has not been much developed, and in spite of careful attention to the difficulties attendant upon the operation, perfect penetration has not been attained. The Obermaier machine is successful for the dyeing of loose wool, but it has not been so successful in the dyeing of cops. The Theis apparatus is much used in Westphalia and Holland for the bleaching of cops; it is also said to be satisfactory for dyeing. The machines used for cop-dyeing do not vary much, and from time to time old types are improved. 


\section{CHAPTER III}

\section{SULPHUR COLOURS AND INDIGO}

Fastness of sulphur colours-Importance as cotton dyes-Their tendering action on cotton-Use for wool dyeing-Machines used for dyeing sulphur colours-Natural and synthetic indigo-Substitutes for indigo-Sulphur blues and indigo.

The first sulphur dye-stuff was Cachou de Laval. Vidal black was afterwards produced by Vidal, and was the first real member of this important class of dye-stuffs, for it was by imitating Vidal's method of production that other sulphur colours were produced. These dye-stuffs were at one time known as " the colours of the future," and nowadays they are recognised as very important cotton dyes. Their brilliancy, deepness of shade, fastness, and cheapness have created quite a revolution in the dyeing industry. Cassella's sulphur blues are well-known colours, and the Hoechst thiogen violets are bright, clear shades. The latter firm claim that their thiogenpurpur was the first red sulphur dye-stuff to be put on the market. It has been stated, however, that other firms had previously had products of a similar nature which they did not deem worthy of being placed on their lists. Of recent years a large number of sulphur colours have been placed on the market, and among these the brands of sulphur blacks have been of special importance. All the colour firms have brought out sulphur blacks of great purity and concentration. Their beauty of shade is beyond dispute, and only in fastness to bleaching powder solution do they fail to compare with aniline black.

The fact that sulphur colours are apt to cause the tendering of the cotton fibre on which they are dyed, has 
prevented them from becoming of still greater importance in the dyeing industry. The tendering does not take place for some time after the dyeing, but in some cases cotton goods, which had been dyed a year previously, had become quite rotten. The cause of the tendering seems to be due to the development of sulphuric acid by the oxidation of the sulphur contained in the dye-stuffs. After-treatment with copper salts accelerates this action and should not be performed. Chroming is recommended, but many dyers object to this extra treatment, saying it is costly and the shade is altered thereby. There is no doubt, however, that chroming, or treatment with some other oxidising agent, as hydrogen peroxide, brings about the full oxidation of the reduced sulphur dye-stuff, and thus produces the fullest shade immediately. To overcome the tendering caused by these dye-stuffs small amounts of certain chemicals, which neutralise the sulphuric acid as it is produced, are sometimes left in the cloth. For instance, sodium acetate is employed for this purpose, and also several patent substances.

Sulphur colours are reduced by sodium sulphide solution, and the solution thus produced used for impregnating the material to be dyed. The colour is developed by the gradual oxidation of the reduced solution in the air, and in most cases a change of colour of the material during the oxidation is noticeable. As regards the temperature at which dyeing takes place, this varies for different brands. Many sulphur blacks dye best just below boiling point, but $60^{\circ} \mathrm{C}$. is sometimes high enough. A low temperature is preferable in many cases, since slow oxidation results and the dyeings are more uniform. A slightly faster result is obtained at high temperatures, but at low temperature the cotton is better preserved. For light shades $30^{\circ} \mathrm{C}$. is often high enough, while some of the indigo brands may be dyed cold with advantage. The amount of sodium sulphide to be added to the bath is also of importance, as a quantity which is sufficient to reduce the dye-stuff is not always enough to fix the dye-stuff on the fibre. Since sodium sulphide solution attacks the wool fibre, sulphur dye-stuffs do not find extensive favour in the dyeing of fabrics containing wool. By using other 
reducing agents however, such as hydrosulphites, sulphites, etc., in place of the sodium sulphide, this objection has been somewhat overcome. But it has been found that on using these reducing agents the results are not exactly the same as those produced by sodium sulphide.

For dyeing cloth with sulphur colours the ordinary jigger is much used, but the author also saw jiggers at work in which the cloth was kept wholly immersed in the dye liquor during the dyeing operation. Exposure to the air during dyeing is said to cause bronzy precipitates on the cloth. The addition of a little glue to the dye-bath is said to prevent these bronzy patches, and thus to assist the dyeing on the jigger. In dyeing hanks with these colours, exposure to air during the dyeing operation is also avoided. The Klauder-Weldon machine is used for this operation (see p. 63) in the United States.

The author found that slop padding was taking the place of dyeing for some classes of colours. Every dyer knows the difficulty of dyeing evenly, and in cases of mordanting and developing dye-stuffs on the fibre the difficulties are increased. Tannic acid divides itself fairly evenly over the fibres, so that impregnation in this case is easily accomplished; but in dealing with some of the chromium mordants and other compounds, difficulties arise. Moreover, tannic acid can be fixed in the wet state, whereas some agents must be dried evenly on the cloth before proceeding to the other operations. Difficulties of this kind have led to the introduction of padding. Thus, in Germany and America, mordants were often padded, and then the dyeing done in the strand dyeing machines. In padding, the cloth passes through the liquor to be padded, and is then squeezed evenly by a pair of rollers. One of these rollers is usually of brass and the other rubber covered. Padding with sulphur colours is difficult, because to ensure a uniform result the dye-stuff should be completely dissolved in the padding liquor. In Germany the author found glucose was used as a reducing agent in preparing sulphur colours for padding. The brass roller of the padding mangle was replaced by an iron one, since brass and copper parts are attacked by sulphur colour solutions. 
Printing with sulphur colours is now performed by using special reducing agents for preparing the printing pastes. Cassella's first used caustic soda and glycerine.

Sulphur colours are much used in the United States. For instance, one representative of a German colour works told the author that he had sold a million pounds of colour in a very short time. Sulphur blacks were in great demand. The introduction of these colours has led to changes in the machinery in use ; copper parts have been replaced. Some firms in the United States claim to have the best machinery for dyeing sulphur colours, but whether this is the case or not cannot be determined because these firms resolutely refuse to place their machines on view. Representatives of the colour makers were not allowed to see the machines, and these gentlemen found themselves in the peculiar position of giving instructions and seeing the firm's servants oscillating between themselves and the dye-house.

Sulphur dye-stuffs have nowadays become so important that they have challenged the position of some of the older fast colours. As previously mentioned, sulphur blacks are used in place of aniline black, and we also find that some sulphur blues have replaced the use of indigo to some extent.

Indigo, one of the oldest and still the most important dye-stuff, has a history full of interest. Not only has the natural article had to overcome the opposition of substitutes; this opposition it counteracted very well, until the production of synthetic indigo caused the natural article to become of less and less importance. Indeed, it is only the present war which has caused a renewed interest in this colour. In 1880 the chemical constitution of indigo was declared, an event which led later to the production of synthetic indigo on a commercial scale. A large industry has arisen therefrom, as the following figures show : from 1896 to 1904 Germany's export of synthetic indigo increased in value from 6.4 to 25 million marks, and the latest returns published (for the year 1913) show these to have increased to 40 million marks. As a result, during the years 1899 to 1903 the price of indigo fell 50 per cent. In 1900, Dr. Brunck of the Badische Anilin und Soda 
Fabrik stated that the quantity of indigo produced annually in their factory would require the cultivation of more than a quarter of a million acres of land in the home of the indigo plant. It has been prophesied that natural indigo will be as dead as madder in a few years' time, yet we find it has to some extent held its position in the East, and various Commissions have tried to improve the cultivation in order to increase the production. But there is obviously a limit to which the production can be improved, for plant life does not admit of the thorough comprehension that operations in a colour works do, and the producers of synthetic indigo have the price of indigo and production very much in their own hands.

The crippling of the synthetic indigo industry by the war has been turned to account by the British and Indian press as an occasion to revive interest in the restoration of the natural production. The movement to break the German synthetic indigo monopoly was started in London three years ago, but despite all efforts the vegetable dye failed to make headway. Many dyers and consumers would have lent their support to the restoration of the natural industry, but the crop season was too far advanced to secure an increased output in the near future. In fact, the area under cultivation in India in 1914 was 20 per cent less than the previous year. In 1895-96 exports reached their maximum at 187,337 cwt.; in 1902-3, a period following the establishment of the synthetic industry in Europe, the exports fell to 65,337 cwt.; while in 1912-13 they only reached 11,857 cwt. The United Kingdom has been most faithful among the principal consuming countries to retain the natural dye, although the country's requirements have been steadily curtailed. Among the strongest merits of the artificial dye were its more uniform character and the easier methods of using it. Moreover, the percentage of indigotin in the natural article was found difficult to determine. Wool takes more readily to the natural dye, but while the artificial article does not yield quite so bright an effect, its colour is constant. To encourage the development of the natural industry two proposals have been made: (1) to subsidise the industry in India, and (2) to restore the obligatory 
natural indigo clause in all Government contracts. This clause is said to have been deleted under pressure of the German monopoly, combined with representations on the part of English dyers. The British Government bought up the Indian crop at the beginning of the war. The author thinks that any attempt to permanently bolster up the natural indigo industry will not meet with success. The only way of fighting the Germans in this industry will be by taking up the production of synthetic indigo.

Japan and China have also renewed their interest in the natural article. Latterly these countries had depended almost entirely on German synthetic indigo. Natural indigo production is one of the oldest industries of both China and Japan. Their own needs being great, they do not expect to be able to export any of the natural article.

Besides the competition of the synthetic article, indigo has had other rivals to face. The introduction of the direct cotton colours had little more than an initial effect on the consumption of indigo, because the direct blues of this class of dye-stuffs were not found to be nearly so fast as indigo. It is true that some direct blues are faster to light than indigo, and that some dark shades have the coppery appearance of that dye-stuff, but none have been found really fast to washing. Attempts have been made to increase the fastness of these dye-stuffs. The fastness to light was improved by an after treatment with coppersulphate solution, and in some cases the fastness to washing was improved by treating the dyed material with a weak solution of potassium bichromate; but still the fastness to chlorine was in few cases good. This fault was also evident in the case of indigo-blue dye-stuffs belonging to the basic class. Metaphenylene blue, Indamine blue, Janus blue, Indoine blue, and Diphene blue had certain good features, but were found wanting when submitted to the action of weak bleaching-powder solution. Naphthindone blue resembles indigo very much in aspect, reflex, and in being exceedingly fast to light, washing, and acids, but again the fastness to chlorine is not good. In order to increase the fastness of the direct cotton dye-stuffs, diazotising and developing on the fibre was introduced, and in this way many good imitations of indigo were produced. 
Diamine blacks and blues, Naphthylamine black, and certain Benzo blues diazotised and developed with beta naphthol and other developers have been much used for producing indigo shades, but again the fastness to chlorine was not found as good as that of indigo. Alizarine blues and the alizarine cyanines have also substituted indigo in many cases. As regards the newer vat blues, there is no doubt that these colours are used for purposes for which indigo was formerly used; but the high price of these colours restricts their application. Moreover, these dyestuffs are found to "bleed" into white cloth (and more so than indigo itself) when boiled in caustic-soda solutions. This defect is particularly noticeable when the caustic-soda solution contains matter which has a reducing action. But of all the colours which have been used as substitutes for indigo from time to time, sulphur colours are the most important. Sulphur blues, which are exceedingly fast to light and washing, are now supplied by the different colour firms ; in some cases the fastness to chlorine is fairly good, but this defect is their great disadvantage. The first sulphur blue was introduced by the Society of Chemical Industry, Basle, and was soon followed by Cassella's Immedial blue $\mathrm{C}$; the patents for the two colours were owned conjointly by the two firms. Other sulphur blues were soon put on the market by the other colour firms. In America, sulphur blues are much used for warp-dyeing and for piece-goods. It is found, however, that these colours are not usually very fast to alkaline boiling.

In spite of the contentions of the makers of sulphur blues, there is no doubt that for producing indigo-blue shades indigo itself is the cheapest and the fastest dyestuff to use. At one time the firm of F. Bayer supplied patterns of cloth dyed with (1) indigo and (2) sulphur indigo blue, saying, "Please test the comparative fastness to washing and boiling." But the fastness of indigo depends largely on the method of dyeing, for if a strong vat be used and few "dips" given, the result is not so good as in the case of the employment of a weaker vat and giving more "dips." This remark also applies to Cassella's statement in their book on cotton-dyeing, that indigo loses in depth of shade every time the material is 
washed, whereas some sulphur colours do not. If pieces of cotton dyed with indigo and with sulphur blue are exposed to the light, it is found that the former fades more rapidly than the latter; in fact, some sulphur colours improve in shade after a short exposure to the air. On further exposure, however, the conditions are reversed, and this distinction is apparent after a few days in summer or a few weeks in winter. Moreover, it is noticed that, although the indigo becomes lighter in colour, it remains the original tone, whereas the sulphur blue, whether it be after treated or not, fades to a greyish shade. Sulphur colours, unlike indigo, are not definite chemical compounds whose constitutions are known to chemists; and as most of them are probably mixtures, it cannot be expected that, as they are gradually decomposed by sunlight, the residual unaffected colour will be the same blue substance as composed the original dye-stuff. In order to emphasise the marked difference between indigo and sulphur blues under the action of light, the Bayer patterns mentioned above were exposed. After fourteen days both were still good in shade, but after forty days there was a considerable distinction in favour of the indigo ; the indigo had remained a light blue, whereas the sulphur colour had become a dirty red-grey shade. The supporters of sulphur colours assert that the tests mentioned are too searching and that the fastness is good enough; but such statements do not satisfy the dyer, since for blouses, shirts, and other articles, colours are required which fade in an agreeable manner. Indigo alone does this, and many German dye-works have found this out to their cost, a return to the indigo vat being the consequence.

Vat dye-stuffs, which have been produced in large variety of recent years, are extremely fast and much used for woven goods. Thioindigo red was put on the market by Kalle \& Co. in 1905, and since that time the Ciba and Algol colours have provided many ather shades. These colours, along with the Indanthrene series, are dyed like indigo and are very fast, but because of their expense are not usually used for heavy shades. 


\section{CHAPTER IV}

\section{MERCERISING}

Invention of the process-Hank-mercerising machines-Piece mercerisingCooling of the lye-Recovery of the caustic soda-Recent scientific work on mercerising.

Mercerising is a process of treating cotton with concentrated caustic-soda solution in order to give it a silky lustre and feel. The effect is not merely produced by the action of the caustic soda on the cotton, but either (1) the cotton must be kept stretched during the action or (2) it must be stretched to the original dimensions after the action. The soda causes the cotton to become transparent, to swell, and to shrink considerably; by resisting the shrinkage the lustre is produced, and, by washing the soda out of the cloth while the cloth is stretched, the cloth does not afterwards shrink when the stretching force is removed. Mercer in 1844 noticed that caustic-soda solution caused cotton fibres to become transparent, to swell, and to shrink; he made use of his observation in the production of " crimped" cloth by printing caustic soda on cotton cloth. Lowe in 1889 discovered that cotton in the stretched state became more lustrous when treated with caustic soda and washed while still stretched. $\mathrm{He}$ patented this method of lustring, but allowed his patent to lapse. In 1895 Thomas \& Prevost repatented Lowe's idea, but the patent was disputed and became void. Experience has shown that for all classes of mercerising, caustic soda of strength not less than $55^{\circ} \mathrm{Tw}$. should be used, that is, in the mercerising mangle, and the caustic soda should be kept as cool as possible during its action on the cotton. Numerous patents have been taken out 
for adding different substances to the caustic soda to increase the shrinkage produced in the cotton, but all these have proved worse than useless. Progress in the art of mercerising has turned to the mechanical rather than the chemical side.

In all mercerising machines the question is the opposing of the force which tends to make the cotton shrink, for if shrinkage takes place the lustre suffers.

Cotton can be mercerised as (1) loose, (2) hank, (3) warp, and (4) piece. The mercerising of loose cotton does not seem to have yet advanced from the experimental stage. Different means have been suggested for gripping the fibres and thus preventing them from shrinking, but this has been found a difficult matter.

For yarn mercerising there are many machines. The principal German machines which the author saw at work were : (1) Kleinewefer's, (2) Haubold's, and (3) Bemberg's. The Kleinewefer mercerising establishment is said to be the largest of its kind in the world. Their hank-mercerising machine works on the centrifugal principle. The hanks are put over a perforated drum and enclosed by another drum. This drum is connected by a shaft to an exactly similar drum at the other side of the machine. Three hundred kilos of hanks are placed over each perforated drum, the outer drums are fixed over the hanks, caustic soda is admitted to the inner perforated drums, and then these drums carrying the hanks are made to revolve by means of the shaft. Centrifugal force causes the caustic soda to pass through the perforations of the inner drums, through the hanks and into the other drums. From these it passes back to the storage cisterns. The inner drums, being stoutly built, resist the shrinkage of the hanks, and in this way lustre is produced. The pipe is then disconnected from the caustic-soda cisterns, and water is let into the inner drums to wash (again by centrifugal action) the hanks. The hanks after being taken out of the drums are further washed in vats by hand. The caustic soda after being used in the machines, of which there are many in the same room, passes into a trough in the middle of the room and thence to a reservoir, where it is kept up to strength. The wash-water is used for 
dissolving the solid caustic soda used for making up the solution in the reservoir. The time allowed for the action of the caustic soda on the cotton was five minutes, and the lustre produced was seen to be very good. It is important to note that the economy of the caustic soda was also said to be good. Most of their mercerising was done in the grey state. Messrs. Kleinewefer informed the author that they were not allowed to sell their machine in England, as their patent rights were bought by an English association of manufacturers.

The Haubold hank merceriser is very much used in Germany. It consists of two sets of heavy stretching rollers arranged in pairs, one over the other, and over each pair the hanks are placed. The distance between each pair is regulated so as to give the hanks a slight stretch, and then the rollers are caused to revolve. In this way the hanks are made to move round and round. The lower roller is then placed in a trough containing the caustic-soda solution, so that the hanks pass in and out of this solution many times. Shrinkage is prevented by the heavy rollers. After the hanks are thoroughly impregnated by the caustic solution, the caustic-soda trough is removed, and the hanks washed by jets of cold water while still stretched over the rollers. To economise the caustic lye, the upper roller is provided with a squeezing roller which, pressing against the hanks, causes as much caustic lye as possible to flow back into the trough. The machine is made very strong in order to withstand the great shrinking force exerted by the hanks when treated with the caustic soda. At the back of the machine there is a similar set of rollers which have the same position and are continuations of those at the front. The machines are made generally with twelve pairs of rollers, and have a capacity of from 500 to 1800 pounds of yarn.

Bemberg's have supplied their hank merceriser to Germany, France, and Italy. It is much like Haubold's in principle, the hanks being stretched over heavy revolving rollers; but there are four tanks or troughs, each containing two pairs of rollers. These tanks are joined together by pipes, another set of pipes connecting them with the supply of caustic soda. 
In a very efficient American hank merceriser the principle is the same as that of the Haubold, but in this case the rollers move along longitudinal paths, the upper and lower rollers describing endless paths while carrying the hanks. The hanks are put on the rollers at one end of the machine, spurted with caustic soda while passing through the machine, and washed towards the end of the passage while still stretched. At the farther end of the machine the hanks are removed, so that the operation is a continuous one. The machine is the same at the back as at the front, and its removable glass sides show what is taking place within. The driving is very powerful. This machine is very suitable for large production and is used by one of the associations of manufacturers in this country. In the Cohnen machine the hanks are arranged radially on a revolving frame, in a somewhat similar manner to the method applied in the Klauder-Weldon machine. It was thought that the lustre would be increased by alternately stretching and releasing the hanks while saturated with the caustic-soda solution, but it was found that no better lustre resulted. With all these hankmercerising machines it is found that some hanks have been less affected by the process than others, and in this case the hanks with the poor lustre are treated again in the machine.

With high perfection in mercerising machines, inventive skill has turned to the saving of the caustic soda. The hanks are well squeezed by auxiliary rollers and then washed on the counter-current principle. The washwaters are, sometimes used in the bleach-house, and in other cases they are evaporated and the caustic soda used again. Sometimes the first wash-waters are re-causticised and evaporated and the weaker wash-waters used for boiling purposes. Hot water and direct steam are sometimes employed to remove the last traces of caustic soda from the material, but it is said that by such a procedure special effects are produced. The swelling of the fibres is said to be less than when cold water alone is used for washing; consequently the threads occupy less space, and the material has a harsher feel.

The mercerising of cotton warps is performed on the 
warp slasher or warp-dyeing machine, tension being produced by allowing the rollers to travel at different speeds.

Machines for piece mercerising may be divided into two classes : (1) those in which penetration is carried out on one machine, the cloth made into a batch and stretched on another machine; and (2) those in which penetration is carried out on one machine, and the cloth stretched on the same machine. At one time many machines worked by method (1), but nowadays most mercerising is done by the second method.

Many different ways have been tried for gripping the cloth and preventing it from shrinking under the action of the caustic soda, but in most cases it has been found that the stenter has to be finally used in order to bring the cloth out to the desired width. It has, therefore, become evident that the stenter is the best machine to use to keep the cloth stretched while under the action of the caustic soda. Cloth has to be turned out by the merceriser at the original width or at some definite width, and this necessity has led to the almost universal adoption of the stenter for piece mercerising.

At one works in the United States the author found cotton pieces being run in the rope form through strong caustic-soda solution, then washed and bleached in the usual manner. As a final operation the goods were stentered, and in this way a fair gloss was obtained, although the width was not fully recovered. One man attended to six mangles in which the cloth was saturated with the caustic lye, so that the labour cost was not high.

The most noteworthy machines not using the stenter for stretching purposes are Schwabe's and Jeanmaire's. In the former, the cloth is passed through caustic-soda solution contained in a trough, then between a pair of squeezing rollers, and finally on to a series of large and small rubber-covered cylinders. These cylinders are so arranged that the cloth is continuously in contact with one of their number, each cylinder tightly pressing against those adjacent. The cloth has thus little chance of shrinking, and the suction of the cloth against the rubber acts 
in the same direction. The cloth is washed while still stretched by passing through troughs containing water, so that the washing is simpler than on the stenter. This machine gives a good lustre, and the shrinkage is said to be only half an inch in a 27 -inch cloth. There is no danger of selvedge damages as on the stenter, and no danger of producing a raggy cloth by overstretching. Schwabe's machine has turned out high-class goods for many years, and has sometimes been worked night and day. Judging from its structure and the number of rollers its initial cost must have been heavy. The machines of Ecob and Hasslacher are similar in action to the one just described.

When using the stenter for mercerising, the great shrinkage force, resulting from the action of the caustic soda on the cloth, necessitates caution when fine cloths are treated. In Schwabe's machine, where the tension is distributed over the whole of the cloth, there is no danger of tearing, but on the stenter the cloth is liable to give way at any weak part. In the machine for which Jeanmaire took out his first patent, the cloth was held by rollers, his device being similar in this particular to Schwabe's machine ; but afterwards pins were used to hold the cloth. The author saw a Jeanmaire machine at work with the firm of Koechlin Frères of Mülhausen. Its output of fine cloth was five miles per day. The cloth passes through the caustic lye on to the round surfaces of cylinders, whose surfaces are covered with a large number of pins. These pins hold the cloth and prevent it from shrinking. Three cylinders are used for this purpose, the cloth passing over each. These machines are at work in Germany and America, and are very suitable for the mercerising of fine cloths. A new French machine stretches the cloth by means of a series of curbed bar expanders. The rinsing with water takes place directly the cloth leaves the impregnating mangle. The expanders are placed both above and below the cloth, and the washing is on the countercurrent principle. The cloth is squeezed in passing from one wash-box to another.

The piece mercerising, done at Bemberg's of Barmen, had at one time such a reputation that English manu- 
facturers were anxious to ascertain their methods of production. They use the method of "batching" after impregnating with the caustic lye. A tight batch is made which after standing some time is taken to a stenter close by, passed through and at the same time washed, treated with acid, and again washed. The machines are made in various sizes, and with 6 H.P. they claim to mercerise 16,000 yards of cloth in ten hours. There was a large plant attached for cooling the caustic soda, but at the time of the author's visit it was not in use. In fact, icecooling plants, which were at one time used, are now seldom seen. Impregnating troughs, however, are made with a double jacket, so that if necessary the lye in the trough can be cooled by passing cold water through the jacket. In order to produce uniform mercerising it is necessary to keep the caustic soda at a fairly even temperature and not above $65^{\circ} \mathrm{F}$.

It is impossible to obtain a good regular lustre on cloth which has not been thoroughly "boiled out" before mercerising. During the short time the material is in the cold caustic lye, unboiled cloth does not soak up the lye very well, and uneven mercerising results. This defect is usually shown up if the cloth is afterwards dyed or bleached, for the properly mercerised parts take up more dye-stuff and are more affected by the bleaching liquor than the parts which have not been thoroughly impregnated by the caustic-soda solution. Bad mercerising can be corrected to some extent by remercerising as previously mentioned in the case of yarn mercerising.

When one has experience of mercerising a certain class of goods on the stenter, one continually alters the machine until the best results are achieved, and it is only after many trials that perfect results are obtained. In this connection, it is interesting to recall the observation of Hübner and Pope that the greatest shrinkage takes place with caustic soda at $45^{\circ} \mathrm{Tw}$. If lye of $55^{\circ} \mathrm{Tw}$. be used, then on washing, that is at the first spirt pipe, sudden shrinkage takes place, or at least the tension is so great that careful watch must be kept. The author came across a machine in the United States which had been altered after repeated trials made by the managers. After impregnation in the ordinary 
mangle, the cloth passed on to the stenter in the usual way. After a short passage, and when still far from stretched to its full width, the cloth was sprayed with the strong caustic lye used in the mangle. This arrangement was apparently to dispense with the use of two impregnating mangles, and the idea was to give the cloth another treatment with the caustic soda after the first dose had had time to penetrate the cloth. After this treatment the stenter gradually widened, and when the cloth was at its full width there was a break in the stenter. The cloth then passed between two squeezing rollers, and again on to the stenter, where it was washed. The manager stated that this arrangement was the best they had used, and they had tried many others. The cloth was certainly a long time in contact with the caustic lye, so that penetration was evidently good. The author thinks, however, that two mangles, as often used, would have had a similar effect.

Some works in the United States claim to have mercerising machines which are better than those generally known. Unfortunately these are not placed on view. It may be that they have some special devices for overcoming mechanical difficulties, but the merits of these machines are probably exaggerated. At one American works the author was shown everything but a new mercerising machine and a machine for dyeing sulphur colours. Both these were regarded as being of special importance, but the partial mobility of labour has probably revealed their "secrets" by now.

In the Gruschwitz machine for piece mercerising, the space taken up by the stenter is reduced by arranging part of it over the lye mangle. One part of the stenter passes wholly through warm water to wash out the caustic soda. The space required for the horizontal stenter is reduced in the Weisbach machine by adopting a vertical arrangement, the cloth being stretched on a circular chain stenter the two side-wheels of which have axles which are not parallel. The cloth after impregnation enters the clips where they are nearest together, and as the wheels revolve the cloth is carried round and stretched. The returns for Crefeld and district, at the time of the author's 
visit, showed that the business of mercerising was not increasing and that they were losing some of their markets. The export to the United States had fallen off, as the Americans are now mercerising their own yarns, often buying them from England. At the same time, South America and East Asia had by increased demand partially made up the deficiency. Competition from all quarters is lowering the profits in this branch of the trade.

On the scientific side of the subject, work has been done by Hübner in contradiction of the early work of J. H. Gladstone on the action of caustic soda on cotton. Gladstone maintained that cotton combined with caustic soda, when immersed in a strong solution of that substance, to form definite compounds called "soda celluloses," while in weak solutions the caustic soda had no such action. Since that time the term "soda cellulose" has had a recognised meaning; but further work has tended to show that such compounds do not exist, and practical experience also points in the same direction. It has been shown that cotton absorbs caustic soda from all strengths of solution of that substance in water, and that there is no dividing line as suggested by Gladstone. Great interest was taken in the statement, which emanated from Germany, that cotton takes up considerably more caustic soda from a soda lye which has been saturated with common salt than from a lye of the same concentration containing no salt. It was stated that 100 grms. of cotton when immersed in a soda lye containing 12 per cent sodium hydroxide absorbed 8.4 grms. of the hydroxide, whereas from a 12 per cent solution which had been saturated with salt, as much as 14.5 grms. were taken up. It was thus thought that salt would supplant some of the caustic soda used in mercerising, and thus economise this expensive chemical. These statements were tested both on the large and small scale, when it was found that the effect of adding common salt to a 12 per cent soda lye was to decrease its mercerising power instead of increasing it. When the lye was saturated with salt, the mercerised cotton produced had not the same lustre or the same attraction for substantive cotton dye-stuffs as cotton mercerised under the ordinary conditions. Moreover, on allowing the cotton 
to shrink while in the lye, it was noticed that the shrinkage produced was not so great when the lye was saturated with salt. Large-scale mercerising showed that the addition of salt to the lye had no practical value.

It is known that mercerised cotton absorbs more substantive dye-stuff and more moisture from the air than ordinary cotton does. The present author further showed (Journ. Soc. Chem. Ind., 1909, xxviii. 188) that the moisture absorbed from the air by mercerised cotton increased as the strength of the lye used in mercerising increased, and was approximately proportioned to the amount of substantive cotton dye-stuff and of iodine taken up from solutions of these substances by the samples of cotton mercerised with different strengths of caustic soda.

As regards the testing of cotton for mercerisation, two methods were described by J. Hübner (Journ. Soc. Chem. Ind., 1908, xxvii. 105) while the present author was his assistant and demonstrator in the Department of Dyeing, Manchester Municipal School of Technology. Of these methods the author has found the following very serviceable in practice. The pattern to be tested is first freed from finishing material and then immersed, for about one minute, in a solution of 20 grms. of iodine in saturated potassium iodide solution. It is then taken out and washed in a test-tube with cold water. Mercerised cotton retains the iodine for a long time during the washing, and remains of a blue-black colour, whereas ordinary cotton is soon washed white. The author uses the iodine solution over and over again, merely pouring it back into the bottle after use, so that the method is a quick one. In comparing degrees of mercerisation by this test, it is necessary to have the samples in approximately the same physical state before testing. For instance, care has to be exercised in comparing two patterns one of which has been calendared many times and the other not calendared at all, for the form of the fibre has some effect on the absorption of the iodine, and also on the surrender of the iodine to the wash-water. After some experience, however, the test is a valuable one to use as a guidance. Cotton cloth is sometimes given the open-width bleach, the boiling being conducted with caustic soda of about $5^{\circ} \mathrm{Tw}$. and after 
schreinering the goods are sent out as mercerised cloth. The above test serves to detect such goods. It also shows any uneven action due to bad penetration and, when carefully performed, gives some indication of the strength of the caustic soda used for the mercerising. 


\section{CHAPTER V}

\section{BLEACHING}

Development of the industry-Effect of chemistry-Efficient scouringContinuous scouring-Open-width scouring-Electrolytic bleachingSteeping of goods-The waxes of vegetable fibres-Bleaching without boiling.

BLEACHING is now a very different process from what it was in the eighteenth century. At that time a period of several months was required to bleach a piece of cloth, and in some cases cloth was sent from this country to Holland to be bleached on the grassy plains of that country. For instance, our linen manufactures were sent in the spring to Holland, where they were steeped in potash lye, washed, steeped in buttermilk, laid on the grass for several months, and finally returned in the autumn. Afterwards a very similar process became established in this country, and the goods were no longer sent to Holland. The introduction of sulphuric acid to replace buttermilk reduced the period of bleaching considerably, and the gradual adoption of chlorine compounds eliminated the long process of "grassing" for most classes of goods. The discovery of the bleaching properties of chlorine gas led to Berthollet in $\mathbf{1 7 9 0}$ practically applying this gas to the bleaching of textiles. The method was introduced into England and Scotland, but it was not until Tennant used slaked lime for absorbing the chlorine, and thus manufactured bleaching powder, that chlorine compounds became extensively used for bleaching. By the introduction of chemicals the length of time occupied in the bleaching process for cotton goods was reduced from several months to a few days. 
The industrial system of bleaching has really developed from the domestic system, that is in imitation of the actions of the housewife in boiling the clothes with alkali, washing them well with water, and spreading them in the sun to dry and bleach. Moreover, modern bleaching machinery has gradually been evolved from the domestic articles. For instance, the copper has produced the kier, and the domestic dolly tub, after itself being used in largescale operations for a considerable time, was replaced by the wash-mills and, later, by modern washing-machines. Just as the introduction of chemicals shortened the period required for bleaching, so has the introduction of improved machinery caused a similar curtailment; but it is remarkable that, although the time required for the different operations has been very much reduced, the old process of bleaching has undergone little change. Details have received attention and some improvements have been made, yet the operations of liming, grey souring, ash boiling, chemicking, scalding, chemicking, souring, and washing remain substantially the same. Attempts have been made to curtail the long scouring operations, but these have seldom been successful. Latterly chemistry has been applied to the process itself, but it is remarkable that science has caused little change in the old process. The introduction of the caustic soda and resin boil has certainly replaced lime boiling for some classes of goods, but still the production of market whites is most often performed by the series of operations just described. Many good bleachers are ignorant of the science of chemistry, and as this science has affected them very little they are apt to belittle it and appeal to their empiricism. On the other hand, chemists are apt to ridicule empirical methods. But it must be remembered that a knowledge of chemistry cannot replace experience in the bleaching industry; there are many observations in practice which the chemist cannot explain, and he must proceed cautiously while paying due regard to the teaching of experience, instead of underrating the value of that experience. Textile fibres are complex bodies which are not thoroughly understood by chemists. Even pure cellulose, the basis of vegetable fibres, may be considered 
as one of the enigmas of the chemical world, in spite of the large amount of work which has been performed on that substance. Nowadays, if anything goes wrong in the bleach-house resulting in "tender" goods, the cry is "oxycellulose." The chemist knows that oxygen and cellulose have been present during the tendering action, and he invents a word to explain the phenomenon, or rather to screen his own ignorance. The same may be said of the use of the term " hydrocellulose." The employment of such terms is an indication that chemists know little concerning the nature of cellulose.

Progress in bleaching has recently been principally on the mechanical side. Proper scouring is essential to good bleaching, and this is probably the most difficult of the operations of the craft. All bleachers know the difficulty of obtaining a really effective and thorough circulation of the lye through the mass of the fabric in the kier. Even in the best constructed closed kiers the liquor endeavours to force itself along the walls of the vessel, where the cloth is less tightly compressed and consequently offers less resistance. The remainder of the mass is not so well treated, and the result is that the bleach is lacking in uniformity. It is obvious that those parts lying in the direct path of the circulating lye will be bleached first, while those lying very remote will stand a poor chance. If, therefore, the boiling of the charge be interrupted after a half or one quarter the normal time for the operation, instead of the whole quantity of cloth being in a uniformly cleansed condition, some parts will be perfectly cleansed, others less so, and finally, there will be parts which are only superficially attacked by the lye. For this reason it is necessary not only to employ a higher temperature in order to obtain uniform bleaching, but also to prolong the time of boiling for several hours, whereas for the complete transformation of the impurities of the cotton only a short time is really required.

Various kiers have been introduced to ensure evenness in the treatment, and some of these have met with success. One type has a perforated pipe down the middle and a perforated cylinder lining the kier and at a short distance from its wall. Between the perforations of the inner and 
outer cylinders the lye circulates instead of being injected through the usual non-perforated puffer pipe and showered over the top of the cloth. Kiers of this type the author saw under construction at the machine works of Haubold, Chemnitz and Gebauer, Berlin. In theWalsh-Jackson kier circulation is produced by means of a powerful centrifugal pump. The lye passes from the bottom of the kier into the pump, and then to a cylinder containing a coil supplied with high-pressure steam; the lye is thus heated while in the cylinder and is then forced by the pump back into the kier.

In all boiling operations the principal danger, as before stated, is that of uneven penetration, for in a kier containing two tons of cloth, the lye has to travel through many layers of fabric. This consideration has led to the introduction of scouring and bleaching in the open width. In this system the cloth is never made into the strand or rope form, but is treated the whole time at full width. It can be readily understood that this system gives uniform results and the damage to the cloth is less, since there is less chance of the cloth rubbing against objects in the way during the treatment.

The Jackson-Hunt kier for open-width bleaching consists of a cylinder with a horizontal axis and open at one end. The cloth in the form of a large "batch" is placed on a special apparatus and wheeled into the kier. The mechanism is so arranged that the batch can be unwound, passed over a perforated drum, and rewound into another batch. The cloth is, in fact, wound backwards and forwards in this way over the perforated drum during the scouring operation, and while passing over the perforated drum it is subjected to the action of a spray of caustic-soda solution, which is pumped from the bottom of the kier and thus through the cloth. There is a specially arranged driving gear to reverse the direction of winding of the cloth when each batch has been unwound. The contents of the kier are supplied with steam at a pressure of $60 \mathrm{lb}$. per square inch during the operation, and it can be readily understood that the caustic soda, at the temperature indicated by such a pressure, produces a thorough and very uniform scouring of the cloth. Before placing 
it in the kier the cloth is passed through a special saturating machine. This machine consists of a large perforated drum placed in a tank of caustic soda solution. The cloth passes over this drum, and the soda is forced into it by means of steam which is blown against its surface. The cloth is then wound into the tight batch for placing in the kier.

Gebauer's of Berlin also build a machine of this kind, in which the cloth is saturated with caustic soda and wound backwards and forwards under steam pressure.

The Tagliani-Rigamonti patent continuous kier was invented by two Milan chemists, and the patent rights were secured by Bemberg's of Barmen, at whose works the author saw it in operation. Steam of low pressure is admitted to the chamber of the machine, which stands over the scouring solution, and at one side of the machine and outside the chamber a head of lye is thus created, which produces a kind of seal for the apparatus. The cloth passes through this seal, that is, through the head of lye between the pressure chamber and the wall of the vessel containing the lye, and is plaited down between two endless chains, which travel continuously over rollers. Between these chains the cloth is carried along, being at one time immersed in the scouring solution, and at another steamed by exposure to the pressure steam in the chamber. The complete operations consist of (1) immersion in hot soda lye, (2) steaming, (3) reimmersion in the lye, (4) resteaming, and finally, the cloth passes through the soda lye in the seal and out of the apparatus. The working of the chains mentioned suggests complications, but devices have been introduced to ensure against mishaps. The apparatus takes up little space, and for its size has a large capacity. The scouring liquor is kept thoroughly circulated by means of a pump, being drawn from the bottom of the kier and showered over the cloth, while it is passing through the kier. It is claimed that the apparatus can take up from 2000 to 8000 yards of cloth, and as the cloth, according to its nature, remains in the kier from one to one and a half hours, the machine can scour from 20,000 to 50,000 yards of cloth in ten hours. It is also claimed that the kier is more economical than the ordinary boiling 
process. The machine the author saw and a few in Italy are the only ones known to be in use.

In the Welter (Mülhausen) continuous kier the inlet and outlet of the steam chamber are hydraulic traps, the former containing the scouring lye, while the other acts as a washing-box. It has, however, a small capacity for its size, even though the capacity has been doubled by running two pieces of cloth in together, that is, one upon the other. Moreover, the apparatus being large, it is necessary to keep the steam pressure low, and this is a great disadvantage.

The question of bleaching in the width is as important as ever, but the cost is higher than for the ordinary process of bleaching. The apparatus of Welter, Tagliani and Muntadas (Paris) give good results, the high cost being their principal disadvantage. The Jackson kier is excellent for heavy goods, but here again the cost of the apparatus is high. In considering the merits of these kiers it must be remembered, however, that the Jackson apparatus is different from the others in the sense that it does not work on the continuous principle, and that, unlike the other kiers, a high steam-pressure is used. The Jackson kier undoubtedly produces a perfect scouring of the cloth.

The Edmeston is another continuous kier. It was the first of its kind. It consists of one large tank open at the top, into which another tank is placed open at the bottom. Steam is admitted into the inner tank and causes a head of lye in the space between this tank and the outer one, the outer tank containing the scouring lye. The cloth is passed through this seal of lye between the two tanks, into the lye of the inner tank, and then, by means of rollers, it passes alternately into the steam of the inner tank and into the lye. Finally, it passes out through the other seal of lye. The Tagliani kier is a similar machine, except that it has one seal instead of two, and the cloth is passed along by means of chains instead of by means of rollers. The alternate steaming and lye boiling in the Edmeston kier is very effective in bringing about the scouring of the cloth. The cloth passes at a rate of from 30 to 50 yards per minute, according to the nature of the cloth. The pressure of the steam is of 
course low, but high pressures and temperatures are not essential for good scouring. In fact, high pressures are used in practice in order to overcome the main defects of closed kiers, viz. want of movement. Nevertheless it is obvious that a kier of this kind is not suitable for all classes of cloth, but for some purposes the scouring produced is quite sufficient.

Of new methods of bleaching which have been introduced from time to time, that of Pick and Erban has received attention. They work the material in a 2-percent solution of turkey-red oil containing chlor-soda, and find this method successful for cops and spools. Turkeyred oil and castor-oil soaps have also been added to the scouring lyes to produce more uniform scouring.

Other bleaching agents besides bleaching-powder solution have been tried from time to time, but few have met with success, and bleaching powder still remains the only decolorising agent used to any great extent. Hydrogen peroxide gives an excellent bleach on cotton, but its cost is prohibitive. Sodium peroxide is used for special work. Sodium hypochlorite, made from bleaching-powder solution by adding soda ash and running off the clear solution, is a commercial article and is used in some works. It has the advantage over bleaching powder of being perfectly soluble, so that there is no danger of suspended undissolved particles in the solution. The present author maintains, however, that this danger does not exist in the case of bleaching-powder solution, if this solution be properly, prepared, and if the steeping system of "chemicking" be used.

Bleaching powder is made in large quantities nowadays from chlorine gas, which is made from common salt solution by electrolytic decomposition: chlorine gas is given off and caustic soda solution is left behind. But if the chlorine gas be not allowed to escape, but is mixed with the caustic soda, a solution of sodium hypochlorite containing much common salt is produced. Special electrolysers have been devised to produce hypochlorite solution in this way. In one of these the electrodes are placed in a shallow trough, the space between them being taken up by a series of glass plates covered with platinum-iridium 
wire and standing vertically and parallel in the trough. The salt passes into the trough from a cooled coil, and by the action of the current it is electrolysed to give a solution containing 3 to 5 grms. of active chlorine per litre. Another electrolyser uses carbon electrodes instead of the expensive platinum ones, and produces a hypochlorite solution containing as much as $\mathbf{1 5}$ grms. of active chlorine per litre. The author was at one time much impressed with the idea of making bleaching solution by the electrolysis of common salt solution, and even the failure of a bleaching company using this method did not shake his confidence. Later, however, he had an opportunity of testing the system on a large scale. An electrolyser of the best type was obtained and worked for many months, so as to give it a fair trial. The result has been that the electrolyser is now idle, several experiments having proved unsuccessful. From time to time there was trouble in working it, and the attention required was far greater than in the case of dissolving bleaching powder. It has often been stated that electrolytic bleaching liquor is not alkaline, but the author found that in all cases it was. By the decomposition of the salt, ions of sodium and chlorine are produced: the sodium ions react with the water to produce caustic soda and hydrogen, which escapes, and the chlorine reacting with this caustic soda produces the hypochlorite. This is the simplest explanation of the electrolysis; and chemical equations show that if all the chlorine unites with all the caustic soda produced the solution remains neutral, as it originally was. This complete union, however, depends on perfect circulation in the electrolyser. But in any case the caustic soda cannot get out of the electrolyser, and, as in practice it is found that some chlorine escapes, the result is that the caustic soda predominates, that is, the solution is alkaline. Donizelli stated that electrolytic liquor often contained so much caustic soda that certain stains were produced on some cloths, but in such a case as this the circulation in the apparatus which produced the electrolytic liquor must have been very deficient. Roscoe and Lunt, on making hypochlorite solution by the electrolysis of sea-water, found, on slightly electrolysing it and allowing it to stand, 
the liquor decomposed rapidly. This decomposition was obviously caused by the weak solution oxidising the impurities of the sea-water, for stronger solutions did not decompose so rapidly, nor did weak solutions made by the dilution of stronger ones with distilled water.

On the large scale the author used bleaching-powder solution of $17^{\circ} \mathrm{Tw}$., and such a strong solution contained a relatively small amount of lime. It was found that the bleaching properties of such a solution were identically the same as those of the solution prepared by the electrolyser. Moreover, on using sodium hypochlorite (obtained from bleaching-powder solution by adding soda-ash solution, the bleaching powder being in slight excess) it was found that this solution had the same efficiency as the bleaching-powder solution from which it was made, and also the same as that of the electrolytic liquor. A large number of bleaching tests proved this (Journ. Soc. Chem. Ind., 1911, xxx. 185), and also their rates of decomposition in air were found to be the same. Among the advantages claimed for electrolytic bleaching liquor are (1) the bleached material is stronger; (2) the white produced is more permanent; (3) there is a saving of acid in the subsequent souring operation; and (4) there is less loss in weight. As regards these contentions, the author has shown (Journ. Soc. Chem. Ind., 1911, xxx. 1295) that, no matter what agent be used for decolorising, the cloth must be well scoured, and that it is in the boiling operations and not in the bleaching proper that the principal drop in weight takes place. The tests of the author (Journ. Soc. Chem. Ind., 1911, xxx. 185) showed that the cloth was not stronger or heavier when bleached by electrolytic liquor than when bleaching-powder solution was used. As regards cost of production, it was shown (loc. cit.) that with salt at 23s. a ton the cost of the salt alone was as high as the total cost of the bleaching powder, which the electrolyser seeks to displace. Similarly with current at $\frac{3}{4} \mathrm{~d}$. a unit, the cost of the electricity was equal to the total cost of the bleaching powder. But with cheap salt and cheap current, it might pay to produce bleaching liquor by the electrolytic method. Electrolytic liquor is very suitable for bleaching 
wood pulp, raw cotton, cops and artificial silk, but for ordinary machine chemicking, the trouble is that no electrolyser produces liquor strong enough for this purpose (Higgins, Journ. Soc. Dyers and Col., 1912, 254).

Another item of interest to bleachers is the effect which neutral salts have in retarding the action of the lyes during boiling. It has also been recently shown that dextrose retards the saponifying action of caustic soda. These facts point to the advantage of giving the " rotten steep" before boiling, in order to remove any mineral and other matter used in the sizing of the cloth (Journ. Soc. Dyers and Col., 1912, 254).

The work of Knecht (Journ. Text. Inst., 1911, ii. 22-29) on the constituents of raw cotton is of great interest to bleachers. In the first place he showed that cotton yarn, from which the wax had been extracted was stronger than the unextracted yarn. Since lye boiling takes away much of this wax, it is probably on this account that lye boiling is often found to increase the tensile strength of cotton goods. The present author further showed (Journ. Soc.Chem.Ind., 1914, xxxiii.902) the efficacy of the lime boil, followed by a soda-ash boil, in eliminating the waxes from textile fibres. The lime boil is not a mere survival of the days of empiricism ; it is the best treatment for the bleaching of certain classes of goods. It was noticed, on plunging lime-boiled and soured cloth into hot soda-ash solution, that a large amount of matter was separated from the cloth, and its wax contents were considerably reduced; this fact is probably due to unsaponifiable and saponifiable (now present as fatty acid after the lime boil and sour) constituents of the wax existing together, the allkali producing a soap with the latter and at the same time removing some of the unsaponifiable matter, by disturbing it and producing an emulsion. The soap is produced quickly as the fatty acid is free (unlike the wax in the case of cloth not previously lime boiled), and a concentration of the soap in the neighbourhood of the unsaponifiable wax is produced. In support of this explanation, it is interesting to recall an experiment by Shorter (Journ. Soc. Dyers and Col., 1915, 3), in which he showed that on adding a 
solution of an alkali to a mixture of a mineral oil and a fatty acid, the emulsification was practically spontaneous. He explained this observation as being due to (1) the rapidity of the formation of the soap layer, and (2) the plasticity of the layer. We have these conditions reproduced in the case of the lime-boiled and soured cloth plunged into the alkali solution. Here the wax of the cloth consists of a fatty acid and an unsaponifiable wax, which for this purpose is similar to the mineral oil. Many methods of bleaching without boiling have from time to time been proposed, but the present author (loc. cit.) gives experiments in support of the contention that only by thorough scouring can good bleaching be produced. In fact, if the boiling operations be curtailed, then the impurities of the cloth, which are usually eliminated during the scouring, have to be decomposed by oxidising agents, and figures show that this is a much more expensive method of removal. At one American work cotton goods, principally crash and towels, were found to turn yellow and become "tender" in stock, and an explanation of the trouble was found after a consideration of the method of bleaching. The pieces in the dry state were steeped in bleaching-powder solution of $5^{\circ} \mathrm{Tw}$. for one hour, allowed to drain for a short time, and then washed twice through an ordinary roller washing-machine. They were then mangled and dried. As to the actual cause of the "tendering " of these goods opinions may differ-chloramines may be suggested by some chemists as the cause,-but certain it is that the trouble arose because the goods had not been scoured before the application of the bleachingpowder solution.

Recently a number of substances have been introduced for use in the scouring lyes, in order to remove the waxy matters of the cloth. In some cases carbon tetrachloride, naphtha, and other volatile organic solvents are mixed with turkey-red oil, so as to form an emulsion which does not readily separate into its constituents. These preparations act very well in extracting the waxy matters from the cloth, but their high cost does not admit of extensive adoption. As a result of some tests performed by the author on the effect of hypochlorite solutions on these 
waxes, it was found that they were partially decomposed and chlorine substitution products were formed, which yielded more readily to saponification than the original waxes. This observation explained the fact that on scalding after " chemicking," a large amount of matter is removed from the cloth. 


\section{CHAPTER VI}

\section{GERMAN AND ENGLISH FLANNELETTES}

Use of raising-Cotton blankets-German production-Large-scale production - German and English methods compared-External and internal specialisation-Inflammability of flannelettes.

Cotron, unlike wool, does not grow on the back of an animal, and therefore cannot have the value of wool as a clothing material. Wool is a bad conductor of heat, whereas cotton is a fairly good conductor. But air is also a bad conductor of heat, so that in a "raised" fabric where a mixture of cotton and air is presented to the body the heat conducted away is less than when unraised cotton cloth is used. In short, raising greatly improves cotton as a clothing material. Very soon after arriving in Germany one encounters cotton blankets. These are made in beautiful designs, by raising heavy cotton cloths, and are found on most German beds. The demand for them is therefore great, and a special industry has arisen to supply them. München-Gladbach, the centre of this industry, is a severely industrial Rhineland town, which resembles our cotton city only in so far as it is principally engaged in an industry which deals with the cotton fibre. In and about this town are 400 textile factories employing 42,000 operatives, and also 111 engineering works with 4000 hands. Since there are only 62,000 people engaged in industry in this district, and as no other industry employs more than 2500 hands, it is manifest that textiles are the chief concern of the people of this town. The author visited factories engaged in the production of cotton blankets, and found them distinguished for their economic working. Water-softening plants were often used because 
of the hardness of the water of the district, and were of proved economy. In the preparing-room of one works, Indian cotton and cotton-waste from Darwen and other Lancashire towns were seen being treated, in the first place, by machinery of a Manchester maker. Mule-spinning was used in the preparation of the weft-yarns, and only slight twist was given, because of the subsequent use to which the yarn was put. The cotton used for making the weft-yarns was dyed in the loose state, for if dyed in the cop the soft feel so essential for blanket-making is lost. The chief consideration is the preparation of a good soft oozy weft, and no pains were spared in the manufacture. A $\frac{5}{8}$-inch staple is used and spun by the condenser system. As many as six machines are used in the preparing, so as to produce a uniform yarn and in this way a uniform nap when the cloth passes to the raising machine. The consequence is that, on examining a German cloth, little unevenness in the yarn is apparent, whereas the irregular nature of some English cloths places the finishers at a disadvantage in attempting to produce a good raised article. At the Gladbach works mentioned above warp-yarns were bought from other manufacturers in Gladbach and Rheydt, and many coloured yarns from which grandrelle yarns were made were obtained from Saxony. In the weaving-sheds the Jacquard looms were seen producing a variety of patterns on a coarse, apparently hard, cloth. In the raising operation, the machines have to be carefully watched in order to produce a cloth of the right "feel" and weight. At the time of the author's visit, operatives for this class of work were difficult to get, and although the industry was in a flourishing state there was a scarcity of skilled workers. Belgians, and workers of other nationalities, at one time paid premiums in order to learn the business of such a works as described.

The cloth, before the actual raising, is submitted to a felting operation in a special machine, so as to make the cloth more solid and to improve the feel ; a brushing and steaming machine is also used to improve the finish. By these processes quite a different article is produced than by merely passing the cloth through the raising machine. The superior finish of the Gladbach goods has been attributed to the good quality of the cotton used; but as this 
is a mixture of Egyptian and Sea Island waste with special short staple Indian cotton, success seems to be due to more careful preparation of the weft-yarns. A uniform yarn is produced from cheap material and a uniform nap is obtained on the raised cloth. The nap obtained being short and uniform, the pattern is clear and the danger of firing is small. In England the cheaper flannelettes are raised quickly, a long fibrous nap being produced, which simply covers the interstices between the threads and causes a flame to run quickly over the surface of the fabric. The works described above had little trade with England, the home market being particularly catered for. Some shipping was done, and that through Hamburg. It was noticed that a Manchester firm of colour printers had been enterprising enough to supply their calendars to works in this district.

The specialised factories that have arisen in MünchenGladbach were originally intended for the supply of the large home demand. Large-scale production, however, led to economic working, and after the home market had been supplied attention was turned to the building up of an export trade, which soon assumed large proportions. Before the outbreak of war, trade with England was increasing, and exports were being made to other European countries, as well as to South Africa, British India, Dutch East Indies, Siam, China, Australia, South and Central America, Mexico, Canada, and the United States.

In most cases a large home consumption leads to specialisation and to export. Sometimes the export trade is very profitable and temporarily raises the price at home, but in the long run the output increases, further economies are introduced, and the home price is in consequence reduced. A splendid blanket can be bought for four shillings at München-Gladbach.

In comparing English and German flannelettes the statement was made to the author that, with the improved machinery used by the German firms, it was possible to obtain a finish which could not be produced in England. It was also stated that during the last ten years the German machine had been protected by patents in England, whereas no such protection existed in Germany; that 
high prices had been charged for the English machines and few improvements made; that German makers have been debarred from exporting their machines to this country, and as a consequence the English flannelette trade has remained stagnant. These statements, made by different persons, are certainly not a true representation of the situation, for two of the most prominent of German makers of raising machines have been shipping their machines to this country for many years. It is true that these firms had a dispute at one time over certain patent rights in this country, but when this matter was settled both firms exported their machines to us. German textile engineers have a habit of sending their sons to this country to receive some education and incidentally to exploit their machines ; in this way a son of one of the machine-makers mentioned booked many orders in this country in 1906.

In comparing the flannelette trade in England and Germany it must be remembered, at the outset, that the industry has developed in different ways in the two countries. The German producers of flannelettes spin, weave, and raise their own material, but in England the manufacture is principally in the hands of three specialised businesses, - spinners, weavers, and finishers. Moreover, in Germany the spinning and weaving operations are specially adapted to ensure good raising, whereas in England the different agents for flannelettes on receiving orders for these goods often also receive stipulations as to whose yarn or cloth is to be supplied, and whose finish is to be given to the cloth. Certain English manufacturers produce cloths of world-wide reputation; customers will have these cloths and no others. Similarly, certain works have a reputation for their finishes, and these productions are likewise in demand. It is therefore manifest that the agent has seldom a free hand in the matter, and consequently the spinning and weaving of the cloth used for producing English flannelettes are not specially adapted to suit the raising operation, as they are in the specialised German factories devoted to this class of work. Moreover, were an English firm to adopt the German method and undertake all branches of the manufacture, they would have difficulty in finding customers unless the agents 
received no stipulation as to the make of the cloth and the finish to be used for the production. But although the organisation of the English industry has disadvantages such as those mentioned, it must be admitted that certain advantages accrue from this development. The fact is that specialisation in this industry has become internal instead of external in this country. Specialised finishing works devoted to raising have arisen, and it is because of this development that the English finishers have been able to keep many branches of the flannelette trade entirely in their own hands. Cloths of all kinds pass through the hands of the English finishers, and consequently a large amount of machinery is required, since different cloths require different treatment in the raising operation. These machines have been constantly improved. The Germans treat fewer classes of goods, and therefore do not require such a variety of machinery.

The upright English-made machine has been adopted for certain classes of goods in this country, and it is said to give a closer and less inflammable nap than the usual machines. The 36-roller machine, which was first made in Germany, is now much used in England; a 38-roller machine has been made in France.

Returning to a comparison of the English and German industry we find that some Lancashire works have adopted the German method of putting the cotton through all the processes from the raw cotton to the finished cloth, but this is not general. Internal specialisation has become the practice of the English textile industry, because of the growth and variety of that industry. Manufacturers prefer to send their goods to outside finishers than to put down machines which they themselves could only run intermittently, whereas the specialised finishers, by keeping their machines constantly running, secure the most economic production.

In summary, we find the works at München-Gladbach using our machinery and our cotton-waste to produce an article which is in great demand all over the world. They produce this article cheaply, because of the large scale of production, and because they have specialised production for one class of article. In England we also specialise 
in the production of flannelettes, but our specialisation has developed internally instead of externally. We have specialised in the production of a variety of flannelettes which no other country can excel. But it is because of the very nature of the development of our industry that we find it difficult to compete with the Germans in the one class of article which they make well. There is no doubt, however, that we could produce cotton blankets as well as the Germans, but we would have to do it in the same way, namely, by producing a good low condenser-weft (about 8's) and following up the manufacture carefully as they do.

In the past, however, the Germans were not our only competitors, for Dutch, Italian, and Spanish flannelettes have been sold in this country.

As regards the inflammability of flannelette, it must be remembered that it is only the low cloths with long irregular naps which are very dangerous; goods which are well raised and have a regular surface are very little more inflammable than the unfinished article. Soaking the cloth in certain chemicals decreases the inflammability, and this process has been gradually improved, but unfortunately those people who have not the sense to exercise care when their children are wearing flannelette articles, have also not the sense to pay a little more for the material which has been treated to make it non-inflammable. Hence the complaints of coroners. Moreover, the cost of the extra treatment required to make low flannelettes fire-proof, brings the price of the treated material very near to that of well-raised goods which, as before stated, are not dangerous to wear. 


\section{CHAPTER VII}

\section{THE INDUSTRY IN THE UNITED STATES}

American conditions-Scarcity of labour-Large-scale production-Uncertainty of their industry-Lack of specialisation-Effect of the tariff.

We hear so much nowadays about American progressiveness and about manufacturers "scrapping" old machinery to make way for new, that any information concerning industrial conditions in America is welcome. The author's experience is that America has not much to teach us as regards the dyeing industry. Some machines were seen which are worthy of mention; a few of these have been devised because of the scarcity of labour, since some of the mills of New England could not, at the time of the author's visit, be run at their full capacity, because hands could not be obtained. Moreover, the enormous influx of foreigners does not supply all demands, for the poorest classes of labour are difficult to obtain, and in the summer months colour strainers and mixers are missing from the print-works because of the objectionable smells of acetic acid and other chemicals. In the manufacturing town of Passaic, New Jersey, which has a population of 35,000 , every race of people is said to be represented, and costumes of all nationalities are seen in the streets, while in the New England cotton mills the Irish work-people are being gradually displaced by Hungarians, Greeks, and French Canadians.

The American cotton and woollen industries, and particularly the dyeing branches of these industries, are so different from ours that often different conditions and machinery are necessary. The American home demand is so large that the manufacturers are not so particular as 
we are as to the material they turn out. American people all desire to be well dressed, and as different seasons of the year demand entirely different clothes, a huge demand arises. The calico-printing done is essentially that of the million, and the very best stuff is either imported or made by a few concerns. For instance, the head of one of the largest firms in New England said: "Here we print or dye a whole lot, put it on a train, and send it in the direction of California; it soon sells. In America there is a huge market, whereas in England you have different designs for different markets. $\checkmark$ Yet the English business is surer. A manufacturer knows how much the grey takes for bleaching, how much for mercerising, dyeing, printing, or finishing, and if he wants a turkey-red he knows how much he has to pay the dyer. Reckoning in this way he can arrive at the cost of placing the goods in his customer's hands, and be assured of his profit. But in our case, where we perform the whole of the operations from spinning to finishing, we have to wait until some period of the year when the audit tell us our fate. In America we aim at turning out the stuff cheaply and quickly." Most mills undertake the whole of the operations from the raw material to the finished article, nor does the industry show much sign of modification in this respect. Instead of specialising internally we find the mills extending their scale of operations. For example, the Gera Mills, Passaic, New Jersey, have begun to spin their own yarns for the making of woollen piece-goods for which they have already a reputation. In New England some specialised works are arising, owing to the rather highly developed state of the industry in this part, and managers are hoping for a time when goods will be dyed on commission, as is done in this country. Specialised bleach-works and dye-works have been installed at Fall River, Lawrence, and other places, and English conditions have ensued at New Bedford in the separation of the spinning and weaving branches of the industry.

Moreover, the extent and homogeneity of the American market has led to the production of large quantities of the same material, and has thus brought about the economies incident to large-scale production, and at the 
same time made their industry of a particular and unique character. America is too occupied with the development of her own market for textiles to give much attention to the export trade; but this development is bound to take place, and already some of the New England printworks ship some very fine prints. With the change and increase in the variety of human tastes everybody has sought the best material, and this circumstance has led to the creation of a large class of good imitations in order to meet all purchasers. It is said that in this class of work we are behindhand, and pointing to the foreigner we are urged to adapt ourselves to the change of circumstances. This supposed backwardness in the art of imitation has not, however, always been one of our failings, for, to the former possession of this trait in our characters, America owes much of the fame of her cotton goods in Eastern markets. In the 'sixties we used to send cotton goods to China, which contained more filling than they did cotton, and in some cases they were known even to crack. China clay and other fillings were employed to cater for appearances and also to save cotton, and so much filling was demanded that the finishers complained that the treatment was ruining their machinery. Moreover, mildew was a frequent occurrence in these goods, as at that time an antiseptic was not used in the finishing mixings. After the Civil War, American manufacturers sent out goods which contained very little finishing material, and very soon American drills and sheetings became of world-wide fame. Nowadays we send similar goods and not heavily filled cloths to compete in the same markets.

The high American tariff has in many cases caused foreign manufacturers to establish branches in the United States, and often the machinery and hands to operate it have been imported. But after a time some of these branches have become Americanised and changed in character. The Gera woollen mills is a branch of the firm at Gera, Germany, the employees, machines, and methods having been brought over from the parent works. Titus Salt's of Saltaire have a branch in Bridgeport, Conn., which is in a further state of development, having become 


\section{DYEING IN GERMANY AND AMERICA}

an American concern. Originally it had English employees, but now only the principals are English by birth. Linen thread mills have also been established, which import their material from the parent works and thus escape the tariff. 


\section{CHAPTER VIII}

LOWELL, LAWRENCE, AND FALL RIVER, MASS.

Lowell-The town and its industry-Merrimack Manufacturing CompanyHours and conditions-Turning to finer work-Production of machinesEmployer and employed-Hamilton Manufacturing Company-Arrangement of works-American worker-Lawrence and its industry-Pemberton Works-Pacific and Arlington Mills-Fall River-Water supplyFall River Bleachery-The drying of cloth-Barnaby Works-Bridgeport, Conn.-Salts Manufacturing Company-Klauder-Weldon dyeing machines.

The town of Lowell is brighter than most English manufacturing towns; it is nicely situated in the country, and abounds in amusements of the best kind. There is also a splendid library and a technical institute. The population is 100,000 , and there are forty mills engaged in different branches of the textile industry. Bleaching, dyeing, and finishing are usually performed by the big works themselves, but there is one specialised bleachery. Lowell is well served by two railroads.

The Merrimack Manufacturing Company have the largest mill in Lowell. They manufacture plain and fancy cotton goods, linens, towels, and corduroys. The capital of the company is $\$ 4,400,000$, and 140,000 spindles, 7200 looms, and 21 printing-machines are used in the works. The manager of the printing department came from Manchester, England, and thus was able to compare English and American conditions in the dyeing and printing industry. The firm willingly allowed visitors to go over their works. There was a combination of manufacturers allowing servants of the different works to visit other works in the combination, and this arrangement was found to be to the mutual benefit of the works 
themselves. A man might get an idea from visiting a neighbouring works, and he might use this idea advantageously, without in any way hindering the works from which he obtained the idea. This combination was really a pool of knowledge, and the manager of the Merrimack Works thought it was the best way of conducting industry, since it was useless to attempt to keep secrets. But all the works do not grant the same concession to strangers, as regards looking over their plant, and some firms are becoming very strict and exclusive. For instance, there are two print-works in North Adams, Mass., where rivalry is so bitter that the operatives from one works are not allowed to discuss business matters with the operatives from the other. This behaviour, however, was not approved by other employers in the industry.

In the Merrimack Works no secrets are allowed.

All the mixings used in the different departments are known to the principals, since they consider that they know more about the dyeing, printing, and finishing of their own cloth than, say, a foreman newly introduced from abroad. Some time ago they had a case of an indigo-dyer who refused to give full particulars of his mixings and methods. This man had to be discharged. It is essential that the managers of a works should have full information regarding all the separate processes of the works so as to trace back any faults which might occur, and also to see that as little waste as possible takes place. At the Merrimack Works a book in each department records what is going on therein. The colour-mixing is of course an important part of the industry, and no dyer is allowed to mix his own colour. Moreover, the dyer is not allowed to take any part in the buying of the dye-stuffs, and on receiving his dye-stuffs he is often ignorant as to the firms which have supplied them. The manager said he had never met a dyer who could mix colour; they usually added the dye-stuff to the bath in pinches instead of taking the greatest precautions to ensure perfect solution.

In the colour-mixing room there was a strong smell of acetic acid, and, as stated before (p. 49), it is because of this unpleasantness that the management have the greatest difficulty in the summer months of getting men to work 
in this department. In fact, the scarcity of labour does not only apply to such low-grade labour, but is evident in all branches of the industry. For instance, part of the weaving-shed at the Merrimack Works was shut down at the time of the author's visit because of the lack of operatives, and an American professor told the author that, for the same reason, he could not get a fence put round part of his garden. Yet in all the open spaces of New England manufacturing towns, and particularly in Lawrence, men are seen lounging about and apparently escaping the work. At the Merrimack Works the working hours are $10 \frac{1}{2}$ a day, and a stoppage is made at noon on Saturdays. The manager said that the most successful works were those which employed the highest-paid hands, had the works scientifically managed, and used the latest machinery. The Arnold Works at North Adams, Mass., is the best print-works in the country, and their fine prints command a foreign market. They are an old-established works, which have specialised in fine prints, and have been very successful. Their success, he said, was due to specialisation, the employment of the best hands in the country, good management, and the use of the latest machinery. Only the firm with the latest machinery could survive in the long run, and if a firm makes big profits, these should not be all paid away in dividends, but a portion put to reserve, in order to ensure even greater profits in the future. The best investment is the "scrapping" of inefficient machinery to make way for later inventions.

The manager then spoke of the advantages of specialisation, and this development he regarded as the true road to success. Schoenhof, in his Economy of High Wages, states: "An English paper-mill boasts of making one hundred and sixty varieties, whereas an American mill confines itself to one, and whereas a Salford mill weaves many kinds of cloth they (Americans) treat of few." There is thus a certain kind of specialisation in America; but the manager of the Merrimack referred to the English kind of specialisation, namely, that in which a works devotes itself to one class of operations only.

At the Merrimack Works they recognise that they are treating too many kinds of cloth. They have a reputation 
for their coarse goods, having made these since 1823, but they have found that treating these goods and their fine goods with the same machinery did not pay. They are consequently going in more and more for the finer goods. This trade was the more profitable, and the price of cotton had not so much influence as it had on the cost of production of the coarser goods. At one time the Southern growers combined, and would only supply a certain amount of cotton. The manufacture of their coarse cloths was thereby severely affected, and they had to shut down part of their mills. It is thus seen that American industry is affected as ours is by speculators in cotton. Moreover, while at Chemnitz the author found the Germans much interested in the presidential election in the United States, as they considered this event decided to a large extent the stability of the cotton market.

As regards his own department, the manager at the Merrimack said, that if they took other people's cloth and printed it at so much per piece they might be more prosperous and more certain of some profit. At present they were making an effort to turn out finer counts, and thus to lead to fine prints. Although England has many more spindles than America, yet the latter country consumes more cotton, a state of affairs due to the fact that English yarn has grown finer and finer. Long training and hereditary skill, among other things, accounted for this advantage of the English, and he thought time would bring England and other countries nearer together, certainly England and America closer, and, he hoped, Bolton and Lowell.

At this works there is a mercerising-machine (described on p. 26), and also a machine for giving a slight mercerised finish (p. 24). These machines were built in their own shop, where they employ many men and make most of their own machinery. This system of attaching machineshops to the works was also found in Germany, and serves to show how much more specialised the textile industry has become in England. Those exploiting industries in new countries can speak of the annoyance caused by the lack of machine and other subsidiary industries, and, in order to ensure success, these subsidiary industries have to be built up along with the primary ones. But specialised 
machine-shops are now arising in all districts of the United States, and gradually the conditions are coming to resemble those existing in this country.

They have a Birch (English) soaper and also many copies of this machine, a Mather and Platt ager, and some of the same firm's printing machinery. They are gradually introducing their own machines in place of the imported articles. Beetling was done at one time, but was found too expensive a finishing process for the class of goods sold in their markets. Some of their stentering machines were enclosed in glass and wood frames. These enclosures confine the heat, but at the same time they do not allow the moisture to escape freely from the cloth which is drying on the stenter. But an important point is that they protect the operatives from the heat, and thus ensure their regular attendance. In other stenter drying machines steam-pipes were placed directly under the travelling cloth, and the moisture driven off the cloth was allowed to escape freely. The pipes were enclosed in order to confine their heat, but the cloth was freely exposed. The hot-air drying arrangement was also in operation, but the second system mentioned, namely enclosed steam-pipes and exposed cloth, was found to be the best.

Few jiggers were used in the dyeing department, most of the dyeing being done by padding. A large amount of para-nitaniline red and naphthylamine claret had been done by them, but there was little demand for these goods at the time of the author's visit. Turkey red was too expensive for the American market.

In the past the Merrimack Company have had much trouble with their operatives, but manufacturers have combined and taken a firm stand on some occasions. The manager said that if they once yielded, they would have little peace afterwards. By agreement they shut down all the mills immediately one mill's hands strike, and thus all Lowell is idle. Then the operatives soon surrender, and resume on the old conditions. There was a strike of engravers at one time, and the Merrimack Company, instead of submitting, sent all their rollers to England to be engraved. This action cost them $\$ 300,000$, but they gained in the long run. 
Referring to engraving, he said they were forced to keep a large number of rollers in stock, but, owing to the high price of copper, they took risks and turned rollers down even when there was a possible chance of a repeat order. English firms, having more designs, have to keep a bigger stock of rollers. The Merrimack Company, like other similar firms, makes its own pattern-cards and does its own making-up and packing, but they have outside selling agents. At one time the operatives were mostly English and Irish, but now they are of many nationalities, Greeks and Armenians predominating.

The operatives are encouraged to work up to their utmost capacity. At one time, if a workman returned more than the usual amount of work, the foreman used to stop the excess; but this procedure has been stopped, and the men are allowed to turn out as much work as they can. The machines are run at a high speed, because it would not pay to run them slower. Thus, in the printing department the machines never stop running from 6 A.M. to 5.30 P.M. ; the printer stays by the machine all day, not even leaving it for meals, which are taken at irregular intervals. The American workmen run their machines at the greatest capacity, and endeavour to get through as much work as possible; whereas in England, so the manager said, the Trade Unions restrict output and hinder development. He said the men were too much in league in England, while in America the slightest united movement is detected, and, whenever possible, prevented.

Yet it is difficult to strike a happy medium between the cause of labour and that of industrial development. It must be remembered that other countries are developing towards our position as regards the power of labour, and in the near future it will be found in America that turning a whole town idle from time to time will not solve all the employers' troubles. From time to time we have had instances in this country of the tendency of labour to hinder the introduction of new machinery. This policy is of course a bad one for all concerned, for if we are to keep our industrial position the efficacy of new machinery must be recognised. The development and progress of our industries during the last hundred years have been truly 
marvellous, surpassing by far the progress made in the previous history of the world, but the rapid advance of the United States and of Germany in recent times has perhaps been even more phenomenal. These advances have been due for the most part to new inventions on both sides of the Atlantic, for in countries where labour-saving machinery has not been developed, man has been found in the lowest and the most humiliating state of degradation, and such would still have been his condition throughout the world without its introduction. As regards our own position in industry, there is some truth in the words of the late Sir R. Giffen that there is enough indolence and routine mismanagement in English manufacture to make it desirable in every way to have the stimulus of foreign competition applied.

Returning to Lowell, we find that textile manufacturing companies are granted land at reduced rates in order to encourage industry. As regards the arrangement of the buildings, at the Merrimack Works it was noticed in the bleaching and finishing department that it was the system to devote each floor of a building to a definite set of operations. The actual bleaching was of course carried out on the ground floor, then the cloth passed to the first story, where it was dried and finished, and finally passed to a third floor, where it was lapped and packed. This system is evidently a good one, and is also adopted by a number of German works. At one American works the transference from one floor to another was facilitated by a series of elevators.

The Hamilton Works at Lowell is one of the biggest concerns in the town. The company has a capital of $\$ 1,800,000$, and has 110,000 spindles and 3000 looms devoted to the manufacture of prints, ticks, flannels, stripes, and shirtings. In the dyeing department mordants were seen being padded, and the cloth afterwards dyed on the rope-dyeing machines. Jiggers are very seldom used in the United States, as the dyeing is principally that of large lots, for which purpose continuous machines are more adapted. Moreover, this class of production is economical of labour, as the machines do not require to be continually reset. For instance, in printing a large number 
of pieces of one pattern the same time is occupied in setting the machine as in printing only a few pieces of the same design. The hours are the same as at the Merrimack Works, but they stop their spinning and weaving departments from 12 to 1 each day. On Saturdays all work ceases at 12 A.M. The printer thought that the American worker did not actually work as hard as the Englishman, and as he had worked for a long period in a print-works near Manchester, he was capable of forming a judgment. He said they had few disputes with their hands. The foremen were often English, but the lower classes of labour were principally recruited from new immigrants, and consisted for the most part of Southern Europeans. French people worked in the spinning and weaving departments, but not in the dye-works. At this works automatic counters were noticed in the lapping and packing rooms; girls sat at these machines, and recorded the lengths as they were called out to them. The machine recorded the addition of these numbers, and gave other information regarding them.

Lawrence, situated like Lowell on the Merrimac River, is engaged principally in the woollen and worsted industry, but some cotton is treated, just as is the case in Yorkshire. The Merrimac River has a fine fall at this point, and provides part of the electric power required by a splendid double line of mills on its banks. These mills are said to be the largest in the world, and certainly no place has as great a concentration of factories as exists along the sides of the Merrimac at this point. Lawrence has a population of 63,000 , and over twenty mills engaged in textile manufacture. There is one specialised bleachery and one dye-works for woollen and worsted yarns, that is, one works devoted only to dyeing.

At the Pemberton Works warps and raw cotton were seen in process of dyeing. The superintendent said hankdyeing was declining, as it was too expensive a process. Warp-dyeing is more rapid and requires less labour than dyeing in the hank form. Warps were being dyed with logwood and with iron buff at the time of the author's visit. The machine used was of the ordinary type, the warps passing over rollers through the solution and then between rollers before going into the next box. The same 
machine was used for dyeing with sulphur colours, and a sulphur indigo-blue was seen giving a bronzy indigo shade. The manager said they used very few different colours, but large quantities of each. There were two machines in use for dyeing raw cotton. In one, the raw cotton and the dye-solution were fed into a tank, which overflowed into a long cistern. By means of a series of big rakes, which were driven by machinery, the cotton was dragged through the dye liquor, and finally drawn between two quickly-driven rollers. The speed of the rollers sent the cotton into a basket, from which it was taken and treated preparatory to spinning. The raw cotton was thus fed in at one end of the machine and came out dyed at the other. The arrangement is similar to a machine often used for wool scouring. The Klauder-Weldon raw-stock dyeing machine was also in use. In this machine the cotton is placed in a perforated cylinder, which revolves on another concentric cylinder containing the dye liquor. By this method it is manifest that better dyeing could be produced, but, unlike the other machine, the process is not a continuous one.

The Pacific Mills and the Arlington Mills in another quarter of Lawrence were visited, and found to have laboratories directed by trained chemists; the chemist at the former works had lectured on printing at the Massachusetts Institute of Technology at Boston.

Fall River, well situated at the mouth of the river of that name, is the most important cotton town of the United States, and contains a large proportion of that country's spindles and looms. The river is so named because of its rapid fall, and power obtained from this fall is utilised in the mills. The population is over 100,000 , and sixty mills are engaged in textile manufacture. Of these, two are print-works, one a bleach-works, another a dye-works, and one works does both bleaching and dyeing for the trade. Of the remainder of the works, some dye and bleach for themselves. There are more works in Fall River, which have devoted themselves, as stated, to particular branches of textile manufacture, than in any other district, this development having resulted from the growth of the industry in this town. It 
is seen that as the industry has developed, specialised works have arisen just as they have arisen in this country.

The capital of the mills varies from $\$ 2,000,000$ at the Fall River Ironworks (a textile manufacturing concern with a peculiar name) to as low as $\$ 20,000$. Water for the mills is obtained in this district from small lakes, and it requires no treatment before use.

The Fall River Bleachery, which specialises in the bleaching and finishing of cotton piece goods, obtains its water from one of these lakes. The capital of the Company is $\$ 600,000$, and the daily output 35 tons. Only lowpressure kiers were used for all classes of cloth. Gas or plate-singeing machines were used according to the nature of the cloth. For instance, a cloth, which was to be afterwards coated with varnish for making insulating material, was singed on both sides by means of an arrangement of four singeing cylinders. Blue dye-stuffs were used for tinting, and in one case a mixture of a basic blue and saffranine was used for the goods of a maker of cheap collars. As regards drying cylinders, the manager thought the best arrangement was to have the first cans arranged horizontally and the last vertically. If all were horizontal, too much space was taken up; and if all were vertically arranged, the steam escaping from the damp cloth below affected the dry cloth above. In order to save more space the "cell dryer" had been introduced. This machine is on the same principle as a make used at one time in printworks in this country. It consists of a big box, which contains a large number (about fifty) of small copper boxes or cells. These cells contain high-pressure steam (about $15 \mathrm{lb}$. per square inch), and by means of rollers driven by a motor the cloth is made to pass over the cells. The machine is small and compact, so that not so much heat is lost by radiation as in the case of the much larger drying cylinders. The cells may also be fitted to printingmachines in order to dry the cloth and the blanket simultaneously; in this case they are arranged at any convenient place, and do not take up much space. During the passage of the cloth through the "cell dryer," which is used for drying bleached or dyed cloth, there is so much friction between the cloth and the cells that, on putting 
one's finger to the cloth as it emerges from the machine, an electric spark passes. The machine is suitable for the American industry, where large lots are treated, but for smaller quantities of dyed goods it might be necessary to clean the machine after different colours are run. Yarn can also be dried on it. Englishmen employed at the Fall River Bleachery said they ran their machines much faster than we run ours.

At the Barnaby Works, Fall River, weaving, dyeing, bleaching, and finishing are performed, cotton and silk goods being produced. Yarns were bleached and dyed before weaving, and were always treated in the warp form. Warp-dyeing with indigo was done in the usual way and "chemicking" in vats, the bleaching liquor being circulated by pumps. Dyeing and bleaching were only performed for their own weaving sheds, which were adjacent to the dye-house.

At the Salts Manufacturing Company at Bridgeport, Conn., the principal objects of interest were the KlauderWeldon dyeing machines for hanks. This machine is found in a great many dye-works in the United States, and is very suitable for their industry. It consists of a cylindrical vessel, containing the dye liquor, and a drum, which carries the hanks and revolves in the dye liquor. On the outer rim and at the centre of the drum are a series of sticks, which carry the hanks so that they are radially situated to the drum. During the revolution of the drum, the sticks, on which the hanks are placed, consecutively come into contact with a metal strip attached to the outside of the machine, and by certain mechanism the hank is caused to move round on the sticks. In this way all parts of the different hanks come into similar positions consecutively, and thus even dyeing is ensured. The machine is enclosed, so that when the hanks are out of the dye liquor during the movement, they move in an atmosphere of steam. The dye liquor is first used cold, and then gradually heated up while the drum slowly revolves. When dyeing to shade, there is an arrangement for lifting the whole drum out of the dye liquor so as to test a portion of the dyed goods. Dyeing to shade is difficult with this machine, but is said to become much easier after experience. 
The dyer at the Salts Works said the machine lasted until the metal wears away, and that it saved a considerable amount of labour. When once started it required little attention. The Klauder-Weldon machine is of course more suitable for large than small lots, and is for this reason well adapted to the needs of the American industry. It is built for batches of from 100 to $400 \mathrm{lb}$. of yarn, and is specially adapted for certain classes of dyeing, as all colours cannot with advantage be dyed in it. For instance, it is difficult to dye basic colours, as there is a tendency towards unevenness ; and for first-class work, as self shades, dyeing by hand has to be resorted to. Another disadvantage is that the machine is difficult to clean, so that a change from one colour to another is troublesome. It is most economically used when large quantities have to be dyed one shade. Developed blacks were being dyed at the time of the author's visit. Many of these machines have copper linings, and this circumstance has prevented their use for the dyeing of sulphur colours. To remedy this, machines have now been constructed with iron walls. In these the liquor is filled to the top of the machine, so that the yarn is immersed in the dye liquor the whole time. The pumping of the liquor is troublesome, but otherwise the machines are said to work well.

The Salts Company's works have also a warp-dyeing machine, which is used for dyeing developed blacks; also a five-colour printing-machine. There is a small laboratory attached to the dye-house, in charge of a qualified chemist, who had been at Yale University, and had also spent some time at a German colour-works.

In the United States the print-works and even the spinning and weaving mills sometimes close down during the hottest period of the year. Because their chief market is their home trade, and as there is little buying during the summer months, the manufacturers sometimes find it better to cease producing and sell their accumulated stocks. The work-people are given a compulsory holiday, and find some temporary employment during the time the mills are idle. In this country, because the bulk of our trade is for export, our print-works are better employed than those of other countries. 


\section{CHAPTER IX}

\section{CONDITIONS OF LIFE IN THE INDUSTRY}

Injurious conditions in dyeing-England, Germany and America comparedThe power of the workmen in Germany-The development in America -Wages in the three countries-America's high wages-Short hoursThe German workman and the American.

Is some branches of the dyeing industry the conditions are injurious to health. For instance, during the polishing of yarn and when yarn dyed with chrome colours is shaken, the fine particles of dye-stuff, which are sent into the air, are harmful to people breathing that air. The aniline vapour produced in some operations is also very harmful, as are the vapours given off during Prussian-blue dyeing. Again, the action of "chrome" on the skin during chrome-dyeing might be mentioned.

The operatives in all countries are protected to some extent, and in this country the regulations are the most stringent of all. Precautions must be taken by our dyers when engaged in some of the operations above mentioned; rubber gloves must be worn during chrome-dyeing, and good ventilation must be ensured during aniline-black dyeing. In America, high wages are apparently some consolation for evil conditions; but to enforce restrictions seems a safer policy, since "whitewash and machinery fences cannot be spent on drink or wasted in betting."

Cleanliness is of the greatest importance in the industry under consideration. A large firm of engineers have their machines painted white and the walls of the workshop whitewashed continually. They say it pays them, because of the good effect on the workpeople. Much has been said against modern works having inside walls of white 
tiles, but probably the initial expense is justified when all the advantages are considered. Some American works employ youths to go continually round the departments scrubbing and dusting.

In the larger and newer works the conditions under which the workpeople toil are much better than those prevailing in old works. In some parts of Germany dyeing is carried on in the most wretched places, whereas in other parts the conditions are much better. At Chemnitz and Elberfeld the dye-works are arranged along the respective rivers, and a trip along these is a revelation. At the former place some of the works have been transformed from old houses ; the office and drying departments are in the house part, while the backyard is covered in and used as the dyeing department. The latter section is very dirty and full of steam; dye-baths, hank mercerisers and bleaching apparatus all seem thrown together, and the small space presents nothing but a mass of disorder.

The manufacturing towns of Germany stand in vivid contrast to those in which manufacture is not so vigorous. When the industry is a new one, some attempt is made to organise it with regard to sanitation and beauty; but in old industrial towns the conditions are far from pleasant.

At Barmen and Mülhausen visits to works showed entirely different conditions from those above described. At one works the absence of steam from the dye-house was noticed; it was driven through ventilators in the roof by means of a current of warm air rising from grids in the floor. The works were well ventilated, and had plenty of space and light. In the particulars given above, the subsidiary dyeing industry has developed in somewhat the same manner as the primary textile industries. "The safety, the cleanliness, the ventilation in all the factories show that agitation and legislation, supported by the humanity of employers, as well as the self-interest of workmen, have raised the sanitary conditions to a higher level in the factories than in the homes of even our modern workmen." The above has been said of the cotton industry, but even in this industry we have some survivals of the old conditions. Similarly, in the dyeing branch the first 
examples given may be taken as corresponding odious survivals.

Similarly in America, in the oldest industrial districts the conditions are the least pleasant. In some cases the dye-house was considered as a mere shed out of which the steam escaping from the dye-baths had to find its own way; in other cases machines were crowded together and surrounded by pools of water. But again, the more modern factories are well built and well kept. A comparison between English and American industrial conditions is difficult: an Englishman visiting America in the summer months finds the conditions disagreeable because the intense heat of an American summer accentuates the defects. But the fact that America has, in some cases, such good conditions is a bad thing for this country, in the sense that some of our best workmen are being attracted by the higher wages and better conditions, and their skill used against us. There is, however, little to choose between the conditions prevailing in the works of England and of New England, for the simple reason that the Americans are following in our footsteps as regards the industry and as regards the legislation affecting it. In fact, all the countries are learning that their wealth is to be found in the quality of their citizens, and that improving industrial conditions is a most rational form of patriotism. Nevertheless, although all three countries mentioned possess both bad and good industrial conditions, yet Germany in the instances mentioned possesses the worst conditions of all.

As regards industrial organisations, it is well known that the trade unions in Germany and America are not so powerful as those in England, yet they are growing at a rate which is alarming to many people in those countries. In Germany the workmen take more interest in political effort than in carrying on strikes. The people are overawed by the police, who have the right to close their meetings and interfere in other ways. Strikes are not of frequent occurrence, for the employers are so strong that the workmen perceive the futility of standing out. Occasionally, however, strikes occur. For instance, there was a dispute, lasting a few days, at the Badische Anilin 
und Soda Fabrik (the largest colour-works in Germany), at the time of the author's visit there. The men won and gained a substantial increase in wages.

In the United States agitation is more in the way of spasmodic outbursts than of a continued and organised effort on the part of the workpeople to assert their rights. The mixture of races militates against the union of labour, and owing to the scarcity of hands employers are generally ready to make individual bargains with workmen. There was a strike at Passaic, New Jersey, during the author's visit. Instead of being a stubborn resistance, it merely lasted one day, and was distinguished by its intensity. The rioters paraded the streets, and attempted to prevent other workpeople from resuming work. This action resulted in disorder, which prevailed long enough for all the police and many civilians in the town to receive injury before the firemen restored order with hose-pipes. In this case the rioters were Italians. But the people are seeing the folly of such outbreaks, and more systematic methods, in opposition to the fruitless strike, are being introduced. The employers have strong combinations, as mentioned in Chapter VIII., and take every opportunity of engaging French Canadians or other non-union labour.

A comparison of wages paid in the dyeing industry of the three countries under review is very difficult to make, for the purchasing power of money has also to be considered. It was generally acknowledged that German wages were lower than English, and that the American rates were the highest of the three, both actually and relatively to the price of commodities. Calico printers, who had experience of industry in the three countries, gave the following figures for a calico printer : for Germany $£ 2: 5 \mathrm{~s}$., for England $£ 3$ and for America $£ 6$ per week. English calico printers living in New England stated that the conditions were much better in their new sphere than in the old country; wages were much higher, while necessaries did not cost much more than in England. Clothes, rents, luxuries and amusements were dearer, but for a quiet life the cost of living was similar to that of the old country. But in considering these opinions it must be remembered that an English calico printer is 
offered high wages to cross the Atlantic, not because of any special quality of his work, but because of the information concerning English methods which he brings with him. Moreover, to simply compare workman and workman, as regards rate of wages, is of little use, unless we know how much employment is furnished at the different rates.

Wages in the German dyeing industry show an upward tendency. It is said that in Germany there are not the extremes of remuneration which one finds in this country, the German wages being more evenly divided. This latter development, however, is still more apparent in America, where a man is given any salary, no matter how big, in proportion to his value to his employers. In the United States, if an employer wishes to economise labour he does so at the expense of the lowest-paid hands; he does not begin by discharging those receiving the highest salaries, and for this reason the influx of unskilled labour due to immigration does not affect the higher salaried posts.

From time to time we are told that some European countries are beating us in some special line of industry because they have lower wages to pay. Germany's low wages and longer hours are said to be driving us out of many markets. This may be true to some extent, but it is also true that low rates of wages are hindering the progress of the German workman. He is forced into the rudiments of education, but class distinctions are so rigid in Germany that he finds it extremely difficult to rise to higher spheres of life. His wages are so low that his movements are restricted to narrow limits. On the other hand, in England one finds hundreds of " self-made men" who now occupy the highest positions, and a large proportion of the manufacturers engaged in the textile industries and their branches started as workmen. This class of men is practically unknown in Germany. The opportunities presented to children of poor parents in this country and in the United States make it possible for a child to forge ahead, and in this way the growth of a purely labouring class is impossible. Germany is regarded as the home of education, but one must have means before being 
allowed to enter a university, and for this reason the larger part of the people are debarred from participation in the "splendid educational facilities" of the country.

A shorter working day has been introduced into some industries, particularly engineering, in this country; Germany is following us in this matter at a distance, and in some parts of the United States the people are awakening to the fact that short hours are in the long-run the most economical. A few of the large German works (for example, the colour-works) are introducing the eighthours day and stopping at midday on Saturday. It is said that the United States have the eight-hours day in many industries, but this is not the case in the textile and dyeing industries. 


\section{CHAPTER X}

\section{EFFICIENCY IN THE INDUSTRY}

Dyeing in Germany-Application of science to the art-The position in America-English workmen in America-Empiricism and scienceArrangement of the works-Using up of wastes-Aids to manufactureSpecialisation in the industry-Dye-works and machine shops-High specialisation in England-British pessimism.

As previously mentioned, Germany has some poor specimens of dye-houses, but the tendency is towards the extinction of such places, and larger and better works are arising. Thus from 1880 to 1890 the number of sheds in Germany weaving cotton decreased from 56,000 to 32,000 , whereas the output increased enormously. The dyeing industry shows a similar tendency. Yet it cannot be said that their largest works compare favourably in any way with the huge bleaching, dyeing, and printing works of this country.

As to the state of the industry in Germany at the time of the author's visit, the reports from the industrial districts stated that the prices of dye-stuffs were falling, as were also the prices paid for dyeing. At Chemnitz the prices paid for dyeing silk, wool, cotton and linen showed a steady decline, and at the same period the business of dyeing both silk and cotton was by no means good in Crefeld. This was the case for both black and colour dyeing, while as regards turkey red the demand was becoming less and less, cheaper substitutes being sought.

We have heard much in the past about the application of chemistry to industry in Germany, but the author did not find that the Germans employ more scientific skill in their dye-works than we do. In fact, the wages paid 
by dye-works are too small to attract the best skill; chemists prefer to enter the colour-works, which employ many chemists in all departments.

In dyeing, as in all scientific arts, countries approach more and more to the same state of efficiency. Secrets in dyeing and scouring existed more in the past, and if one country is much ahead in this industry, the circulation of technical periodicals all over the world, and the partial mobility of labour between countries, tends to equalise matters. This process of diffusion is, of course, slow, and the progressive countries gain certain advantages during the diffusion, but let us consider what the position would be if such a fictitious state of equality were arrived at. New discoveries would be made and inventions patented; success would then lie in the judicious use of these inventions. As regards our own industry, there is no doubt we derive some benefit from climate and hereditary skill, but these advantages will not avail us much if we fail to keep up to date and make judicious use of modern inventions.

In the dyeing industry the Americans do not shine. They treat larger lots than we do, and for that reason use some different methods; but in other respects they seem to be just following us at a distance and, by capturing as much of our talent as possible, making that distance as small as they can. Evidence before the Industrial Commission of the United States, 1900, showed that England was practically supplying the demand for trained workmen in the United States. Many Germans are employed in the calico-printing works, but the responsible positions are generally filled by Englishmen. In fact, the author got quite used to managers saying they used to work at certain Lancashire works. Englishmen are also employed as teachers in the textile schools. English machinery is largely used, but more and more American is being introduced as machine shops are being established.

America is exceedingly rich in raw materials. She has splendid supplies of coal, water power, iron ores near the surface and close to coal and limestone, great stores of copper, lead, zinc, corundum, quicksilver, asbestos, asphaltum, nickel, cobalt and kaoline, besides native wood 
of great variety and excellently adopted for manufacture, building stones, slate and marble, besides cotton and wool. These resources must always be considered in accounting for America's position in any industry. The differential advantage produced by these resources is often accounted for in other ways, and in some cases the high quality of her labour has wrongly been given as a cause. For, as regards the textile industries, the labour is supplied by a collection of nationalities directed, as before stated, by British immigrants.

Empirical methods are prevalent in America as they are in this country. For instance, at one American works a man who had no scientific training was seen giving directions to the dyers and dispensing dye-stuffs in a most crude manner. The workmen brought pots and received dye-stuff, by the aid of a spoon, from one or more of a dozen cans. By long experience the man could tell how much he took on the spoon, but he admitted that weighing was the better method. The workmen passed to and fro between their machines and this man, bringing patterns and their pots to receive more dye-stuff, until the right shade was obtained. Weighing out the colour was stated to be too slow for them. The dye-stuff was invariably introduced to the bath in the solid state; either the importance of perfect solution was not recognised or the time taken for such an operation was held of more consequence.

But some of the American dye-works are conducted on scientific lines. At the Arnold Print Works, North Adams, Mass., success is largely attributed to the systematic and scientific working of the mill, while the Merrimack and Arlington Mills, two of the foremost mills in Massachusetts, are thoroughly up to date. The Arlington Works at Lawrence has a fine laboratory, and the chemist has an assistant who was educated at Lowell Textile School. They test all the materials which come into the works, and do washing and other tests of the dye-stuffs. They have a Bradford machine for determining the moisture in textiles. At the Merrimack Works all materials coming into the works are tested, no matter what their source, and the manager said the events of 
the week previous to the author's visit had proved the wisdom of their plan. During the whole time they had received dye-stuffs from one of the largest German colourworks they had found no errors until this occasion, when a keg of rhodamine was found to be decidedly under strength. Previous testing had in this case saved a great amount of trouble.

At the present time one of the principal roads to success lies in using up wastes. Lord Masham said he maintained his house out of his sewer ; and so many things are now recovered which formerly were allowed to pass into the sewers that manufacture has assumed quite a different aspect. This is undoubtedly the department where science can be of use, and where, in fact, it is certainly being of use. In America the treatment of waste products has as yet received little consideration, and wastefulness is an element of weakness in their manufacture. Owing to their huge resources the genius for petty economies has not been developed; the artisan is habitually indifferent to economy of material, and the employer is more concerned with economising labour. On the other hand, in Germany, the success of many of the business concerns has been due to attention to details and the practising of small economies.

In Germany and America one notices that industry receives every consideration. In Germany the State railways assist industry in all possible ways, and in the United States the authorities even allow trains to run down important streets rather than put the railway companies to the expense of laying special tracks. America seems to proclaim that railway development and industrial development must take place together. For instance, coal is found in Massachusetts, but being mixed with earth it is of little value, so that the coal for the factories in that important State has to be brought by rail from neighbouring States. One hears little in the United States about the development of waterways. The Erie Canal, so much used in the past for developing New York State, is only now used for lumber, salt, and coal ; most of the large businesses use the railway entirely for transport. In Germany the principal use of canals is to connect the big rivers. 
In connection with the aid afforded to manufacture in America, it was noticed that a manufacturer was not summoned if black smoke appeared from his chimneystack, for at one time black smoke and even nitrous fumes were seen issuing from chimneys at Passaic, New Jersey. They are not so strict as we are in this respect, but then they have not the congestion of works that exists in this country. As mentioned in another part, grants of land at reduced rates are made at Lowell and other American cities.

As regards the state of specialisation in the dyeing industry of the three countries under review, it has been previously stated that the lack of specialisation in Germany is due to the fact that their primary textile industries and, consequently, their subsidiary dyeing industry are not so large as ours. Whole works do not devote themselves to special branches of industry, and usually a dye-works undertakes a large variety of operations. In America we find spinning, weaving, and dyeing performed by the same works, but in Germany this is seldom the arrangement; the subsidiary industry was founded as a separate industry at the same time as the textile industry itself was established, so that separate dyeing establishments resulted. In Germany one finds, however, that frequently textile machine shops are attached to dye-works. In these engineering departments the dyeing machines, which are used in the dye-works, are made and placed on the market. For example, Haubold's of Chemnitz, Gebauer's of Berlin, Kleinewefer's of Crefeld, and Bemberg's of Barmen, the large textile machine works in Germany, were originally and solely dye-works. In course of time machines were invented in these dye-works to facilitate the dyeing operations, and then these machines were made in quantity in a special branch of the works. In some cases general engineering works in the district were taken over, for the engineering branch of the business was found more profitable than the original occupation. Experience in the working of the machines resulted in the improvement of these machines; but the works mentioned now make not only their own improved and patented machinery, but machinery of all kinds. 
Haubold's of Chemnitz is about the largest textile machine works in Germany, and a dye-works is run under the same direction. Gebauer's of Berlin have a large engineering works, which gradually developed from a dyeworks. Seeing the importance of good machines, and knowing the requirements of the industry, they began to build machines. Later, they bought a works for making woollen machinery. They export machines to all parts, but few to England. Kleinewefer's of Crefeld have a large mercerising department, and a bleach- and dye-works alongside a machine shop, where silk-finishing and other machines are made. Bemberg's of Barmen, a large dyeing and finishing works for piece-goods, absorbed the engineering works of Mommer, and developed it along with the dyeing branch. They also held the Schreiner calendar patent. They make a hank-merceriser, calendars, presses, etc., and used to make a cop-dyeing machine until competition became too keen.

In America a few cases were seen where the dyeing and engineering industries were connected, but this was not general. Some works, from necessity, made some of their own special machines, but in few cases were these made for sale. The Cocheco Manufacturing Company of Dover, New Hampshire, however, make the "cell-dryer," and have put it on the market, but their principal business is the making, bleaching, dyeing, and printing of cotton goods, velvets, and velveteens.

In England, engineering of the kind mentioned is undertaken by specialised engineering works, and is seldom connected with dye-works. As our industry has developed, certain engineering works have specialised in the production of bleaching, printing, dyeing, and finishing machinery.

As previously mentioned, most of the dyeing in the United States is done in the same establishment as the spinning and weaving of the material, and even great developments have not resulted in the dyeing operations breaking away as a separate branch of industry. The tendency in America is rather in the other direction, that is, the works are increasing in size in the direction of selfsustenance. For instance, the Gera Mills at Passaic, New Jersey, have hitherto been known for their woven woollen 
goods, but they have now begun to spin their own yarns in order to ensure a good supply. The different works specialise in the production of certain classes of goods, and they perform most of or all the operations required to turn the goods from the raw material into the finished products. In the same way the Germans make flannelettes as described in Chapter VI. This class of specialisation may be termed external specialisation. In the English textile and dyeing industries, however, another form of specialisation has developed, and this is of an internal nature. The dyeing industry is more specialised in England than in any other country, and to this development success in our various foreign markets is due. It has brought about great economies, so that other countries are striving to imitate our organisation. We have specialisation so developed that one district is known for fine spinning, another for weaving, while in dyeing, one works, or a combination of works, is noted for its turkey red, and another for its blacks. These separate branches of the industry have been developed to such a state of perfection that their productions cannot be equalled by any other country, and it is because of this high state of perfection that our export trade is so great. Specialisation has led to special training in particular branches of industry, and this mode of organisation is absolutely essential to British success. Similarly in the worsted industry we have the wool merchant, the comber, the spinner, the weaver, the piece merchant, the dyer, and the distributer. These operations are often carried on separately in this country, but in the United States mills are seen spinning, weaving, dyeing, finishing, and despatching their own woollen goods. In this country the dyeing is arranged for by the merchants, who send the goods to the dye-works which is known to have specialised in certain classes, whereas in the United States the dyeing is almost invariably done on the same premises as the weaving and other operations. In this country we have realised that in specialisation lies the strength of our industries, because a man concentrating his energies on one particular kind of work does that work as economically and as perfectly as possible. Moreover, specialisation of labour cheapens production, because a 
habit is slowly formed, and we learn the shortest and best way of doing a thing only gradually.

It can be safely said that, as far as the dyeing industry is concerned, the exhortations sometimes addressed to us to reform our methods do not call for urgent consideration. We have been told from time to time that goods have gone from this country to Germany to be dyed and returned to us, and this fact has been often cited as evidence of the eminence of German dyeing. At the same time, however, no mention was made of the works in this country which have been continually approached by Germans and others to undertake work of the kind mentioned. In the case of one particular works, the author knows that German and Belgian goods were treated until the troubles of transportation made the works give up the business; that an Austrian firm had goods treated and was anxious to gain information as to the method of production; and that a Russian firm, after seeing some of the goods which had been treated at the works mentioned, offered payment for a knowledge of the method of treatment. And this was not a case of a special patented process, but only one example (and there are many others of the same nature) of a works having, by careful work and technical excellence, produced goods which no other establishment in the world could improve upon.

Again, the mere fact that the Germans issue a large amount of research work in the technical journals is regarded as a sign of great activity; it must be remembered, however, that much of this work is of little value. If, during research on industrial matters, anything of real value is found out it is not published but protected; the matter which filters through is generally of purely scientific interest only. Moreover, many of the methods which are published in technical treatises are those which have served a previous period, for new and important methods are not usually advertised by those who use them. Most of the German technical publications contain hardly any facts to add to industrial experience. Indeed, so much has been written on technical subjects that there is hardly any justification for new literature, unless it contains some real addition to our knowledge of the subject treated. 
In English works a good deal of research work is carried out, although few of the results get into print. Those of a practical nature are used, and the rest of the material is generally filed for future reference. For instance, at the time of the introduction of mercerising, or it might be said the revival of that process, it is known that much research work was done on the subject at large works in this country. Some of the results were used in industry, and some were of scientific interest only. In later years, many of the same results were found by other experimenters and published for the first time. To those engaged in industrial research there is little gain to be obtained from the publication of results.

What the author wishes to point out is that, although the fact is not advertised by the publication of papers before learned societies, a large amount of research work takes place in our dye- and similar works, and as a result of this work processes are improved, economies are made, and modern methods used. Mr. Hooper, Secretary of Bradford Chamber of Commerce, said that "the very existence of the Yorkshire manufacturers and merchants depends on them keeping abreast with the times. No industry in the world has been subjected to so many changes or suffered so much from the vagaries of fashion as has the worsted industry; no other industry has shown more readiness to throw out obsolete machinery and instal new plant in order to meet the ever-changing requirements of the days, and so long as the Yorkshire manufacturers, dyers, and merchants maintain their present enterprise, inventiveness, and vigour, they have nothing to fear from foreign competition," and similarly Lancashire can hope for the same fate so long as she has her "energetic, self-reliant, and inventive people, and her excellent industrial and commercial organisation" (the late Elijah Helm). "The natural life seems as vigorous as ever, but the utmost energy and resource must be applied in every direction if we are to hold our own" (Sir R. Giffen). 


\section{CHAPTER XI}

\section{INSTRUCTION IN DYEING}

The dyeing industry subject to change-British pessimism regarding education-Appointments for technical students-German technical schoolsEducation of the German workman-American schools - Suggested reforms.

THE dyeing industry is probably more subject to change than any other branch of manufacture. In the first place, we have the changes brought about by reason of the large number of new dye-stuffs and of new classes of dye-stuffs which are continually being introduced; we have changes in the methods of dyeing with the old dye-stuffs; we have progress in manufacturing chemistry, leading to the cheapening of the production of some chemical, which thereby finds its way into the industry and causes a change of methods; and we have changes in the nature of the fibres themselves. When these facts are considered, along with the vagaries of fashion and the peculiar nature of the demand which the dyer has to meet, one concludes that only the most enterprising and progressive dyers can survive.

Perhaps there is no industry in which technical training plays so important a part, and no industry which in future will depend so much on technical skill as the dyeing industry. It is becoming systematic; it is settling into scientific order ; the value of rule of thumb is decreasing; and looking ahead one can conceive of a time when dyeing will be performed by labourers under scientific direction. In the light of these facts it is interesting to record the steps which different countries are taking to supply technical instruction in this subject in order to ensure the survival 
of their dyeing industries. If the books and papers which have been written on technical education are consulted, the definite conclusions that can be gathered are in reality few; the only substantial inference the author can draw from them is that the people of this country are very pessimistic.

If blue-books and the reports of commissions are consulted the reader is confused by conflicting statements. For instance, in the Eighth Annual Report of the Commission of Labour of the United States it is stated that England is ahead of America in that the English employer recognises the superiority of the technically educated apprentice in many mechanical operations, not as regards manual dexterity, but in the comprehension of general principles and in the power of reducing theoretical knowledge to tangible results. On the other hand, the Report of the Moseley Commission called attention to the deplorable apathy of English manufacturers towards technical education, and stated that the American manufacturer was ready " to pour out his money" to assist the progress of technical instruction. From another authority we hear that our shortcomings have received universal assent, but also practical neglect; a Royal Commission, it is said, has roused a feeling of consternation, but has been soon forgotten in that fatal optimism which is the normal state of the public mind. In the same direction a Departmental Committee of the Board of Education reported that in technical instruction Germany and other continental countries were much ahead of this country. And yet we found, a short time ago, a German professor deploring the backwardness of his country in certain branches of chemistry, and holding up to his countrymen the examples of England and Paris as pioneers in these branches of the science.

From these conflicting opinions it is difficult to arrive at a true conception of the position. The author thinks, however, that an entirely wrong impression exists as to the state of technical instruction in this country. Deputations going to Germany to study the German system of education do not get a clear view of the case. They start out with the idea that German institutions are far 
better than ours, and, moreover, while there, only note those things which are actually brought to their notice. Further investigation, however, proves that a wrong impression has been created. Sir William White, in a speech before the Institute of Metals, called attention to the fact that certain Englishmen were belittling our industries and creating a bad impression in the eyes of the world. Truly the national pessimism extends to educational affairs. Whether a country is best urged on by the assumption of an optimistic or a pessimistic outlook is not to be argued here, but certain it is that, as regards educational affairs, most countries put forth a show of pessimism. The author, having had a university training, having been on the staff of a technical institute, having made a tour of the principal industrial centres of Germany and America, besides examining the technical schools in these countries, and having had some industrial experience, is prepared to attempt to turn a spirit of pessimism to one of optimism.

We have been told by one class of critic that English manufacturers are apathetic, and do not appreciate technical skill. But the fact that England holds such a commanding industrial position seems to point out that any fault does not lie with the manufacturers. When the right article is presented, our manufacturers are as ready to snap it up as those of any other country, although naturally few manufacturers are prepared, considering the mobility of labour, to train a man without an assured prospect of profit from the transaction. In educational matters it must be remembered that the direction of instruction is determined by the opportunities available for scientifically trained students in the commercial world. We have here the operation of supply and demand as in industry itself. Professor Ashley has pointed out that this is true of all branches of knowledge. For instance, he thinks that economics will never flourish as a university subject until it is made a subject for examination for some professional career, or connected with business life in some way, as is being done by the founding of the Faculties of Commerce at the universities. Considerable numbers of people might be drawn to universities and 
technical schools by a passing interest, and mere habit might keep many of the students there after the interest has flagged; but certain it is that no study can flourish unless pursued with a professional object. Few students will be found to attend merely for the love of the different branches of knowledge.

So it is with technical education; as it becomes more and more a "bread and butter" requirement, so will it flourish in all communities. For instance, in Germany the polytechnics grant degrees, and on this account have undoubtedly grown in popularity. In Prussia no examinations take place unless certain specified rights, conferring privileges either for future study or for admittance to a certain career, result therefrom. It is a case of no rights, no examinations. An Englishman entering Germany for the first time is surprised to find that so many people understand English. The reason is that English is so necessary for their trade with England and America that there is a certain profit to be obtained from acquiring a knowledge of the language. Again, the exemption from a portion of military service of those who have a certain standard of education is of great benefit in urging many to study. Perhaps it is well for all to remember that examinations are not pleasant experiences, and a student has little inclination towards them if he sees nothing beyond. If in this country more public appointments were offered to university and technical school men possessing certain qualifications, then there would be a very great impetus to study.

But at the same time it must be admitted that the ladder by means of which a poor child can climb from the elementary school to the university is much more complete in this country than it is in Germany, since big scholarships are practically unknown in the latter country, and education divides the people into two rigid classes. This question of the pursuit of education for profit is well brought out in the quotation previously mentioned of a German professor deploring the fact that England and Paris were foremost in certain branches of chemistry. The branches, he specified, were inorganic and analytical chemistry. This is, no doubt, due to the large absorption 
of the German chemical graduates by the colour-works of that country having inclined the study of chemistry to the organic side; the demand for organic chemists has influenced the direction of the study of the science. Indeed, it might be said that the excellence of these colour-works is the cause of much of the talk about superior German methods and systematic German chemical education; these concerns are continually held up to our notice. If Germany were suddenly to lose these works we would hear little more about the excellent business ability and technical education of our rivals.

It must also be remembered in considering all education that many individuals are incapable of seeing the gain to be obtained from submitting themselves to a course of instruction, and that many rate-payers are unable to see the necessity of the high education rate; such shortsighted individuals exist in all communities, and no doubt hinder the progress of thought and instruction. Although in Germany the supply of graduates is determined more or less by the demands of different occupations, it is found that there are many graduates who find difficulty in entering industry, and others who work for small salaries; this of course is the state of affairs in this country, and, in the nature of things, unavoidable. English chemists are found to wander abroad just as German chemists are found within our shores, for some of Germany's best chemists come from Zurich and England, while many more have benefited from a long stay with us.

Of the German schools which are devoted to teaching the practice of neighbouring industries, the one at MünchenGladbach, which is the centre of the cotton industry of Rhineland, is among the newest. This school is somewhat like our smaller technical schools. The equipment and maintenance are provided by local manufacturers, aided by a small grant from the Prussian Government. It is under Government control. The building contains a boiler and engine-house, spinning, weaving, and dyeing departments, a chemical laboratory and lecture rooms. The spinning and weaving apparatus is mostly of a small size. The dye-house contains pots for sample dyeing, besides a few jiggers and small apparatus for dyeing. 
Much of the machinery in the school was made in Lancashire, and it is worthy of note that many of the machines used in the cotton industry of the neighbourhood were supplied by us. The complete course of instruction at the school extends over two years, or four semestres, each of twenty-one weeks of forty-four hours. Students must be over sixteen, and possess a satisfactory general education. Special instruction is given in the production of cotton waste cloths, which are a speciality of the district. The school is designed to assist the local industry, and foreigners are only admitted at the discretion of the director; they are charged at the rate of $£ 40$ a year. Students from other parts of Germany pay $£ 15$ a year. At the time of the author's visit there were 32 day students, 8 of whom were foreigners, and 143 evening students. One noticed that the school was advertised in many of the works of the district.

The Crefeld Fachschule is one of the oldest schools of its kind, and since its establishment in 1896 has undoubtedly had much effect on the German textile industries, and at the same time gained a good reputation. This school is really a combination of a trades school and a school of chemistry, because it was recognised at its inception that a knowledge of pure chemistry is essential to the study of the dyeing and other branches of the textile industries. Lectures on physics, inorganic, organic, and physical chemistry, and patent legislation are given. Some of the chemical laboratories are used for research connected with the textile industry, and other rooms are fitted up for the usual chemical requirements ; in a small experimental dye-house trials are made on a small scale. The large-scale apparatus includes hank-dyeing becks, raw cotton dyeing apparatus, centrifuges, silk-finishing machinery, and printing-machines, driven by hand and power. The director of the school receives a salary from the town of Crefeld, and he is allowed to do private work. At the Chemistry School of Mülhausen, instruction is given in pure and applied chemistry, but no large-scale apparatus is used. The school aims at giving special chemical instruction to those entering the dyeing industry.

Chemnitz, usually called the "Manchester of Saxony," 
because it is the centre of the cotton industry of that country, has a department of technological chemistry at the Staat-Lehranstalt. Here the teaching is of a theoretical kind ; instruction is given in dyeing, but no large-scale apparatus has been installed. At the Charlottenburg Technical High School there are a large number of special chemical courses. The chemical department takes up a large portion of a large, handsome building. There are many research laboratories. Yet the author thinks that there is no justification for the exaggerated statements which have been made concerning this school. A mixture of an art and a technical school, it suffers by comparison with the Schools of Art and Technology in Manchester.

Karlsruhe has one of the finest of German technical high schools. Here pure and applied science is taught and degrees are granted. There is some dyeing in the district, and this industry is catered for at the school. Thus a research on the fading of colours under the light of the Cooper-Hewitt lamp was being carried out, at the time of the author's visit, by Professor F. Haber, and a new dyeing laboratory was projected.

In Austria one finds that instruction in dyeing is not well provided for, so that many Austrians visit other countries for their instruction. The Königliches Österreichisches Gewerbe Museum, Vienna, is a higher grade technical school which is supported by Austrian manufacturers and receives a Government grant. It is mainly used for the teaching of dyeing and printing from a theoretical standpoint. There are various testing departments. The only school in Austria devoted to the teaching of practical industrial methods is the Reichenberg Staats Gewerbeschule, which has been designed on the Crefeld pattern and contains a school of dyeing. Very low fees are charged and sometimes free tuition is given.

From the above short description of a few continental schools it must be evident to those who are familiar with the English schools of the same kind that at least, as far as equipment is concerned, England does not occupy a second place. It is true that many of the German technical high schools attract large numbers of students, but this is not the case with schools of the Crefeld type. The 
Crefeld school itself had, in 1906, seventy students, which number, however, dropped to forty-five in the following year when the fees charged to foreigners were raised. The München-Gladbach school was for some time without any students at all. Sorau, a town in Brandenburg near the borders of Silesia and Saxony, and therefore quite near the dyeing industry of this part of Germany, built a school of the Crefeld type at a cost of a million marks and equipped it like a university, with a fine laboratory and machinery for bleaching, dyeing, printing, and finishing. At one time there were three instructors and six students, some of the students being foreigners. Further, we find that in Germany the schools are not very particular as to the quality of the student they admit; they make an effort to get as many as possible. It has been said that students from the high schools pass through the schools of the Crefeld type before entering industry; but the author found that such cases are exceptional, as these men have other outlets for their training. However, a student from a technical high school does sometimes find his way into a trade school, and then the authorities show their delight; they make no mention of men of inferior training that they accept. The technical high schools themselves are known to receive in their specialised technical chemical laboratories, students who have had little previous chemical training. Further, the author recalls the case of a Hungarian who had passed through one of the best schools of dyeing in Germany taking the full course, and who yet lacked even the most elementary knowledge of chemistry. He could not have known very much when he was accepted.

The volunteer departments of the German colour-works are now much patronised in place of the schools mentioned, but it must be remembered that the training obtained in these departments, although good, cannot become a substitute for attendance at technical schools : the experience is of a different nature, and can only be supplementary to technical instruction.

As regards the training and education of the workpeople engaged in the German textile and allied industries, the situation, the author found, is very similar to the state 
of affairs in this country. The employers are inducing the people to attend technical classes, and the law is applying as much force as it can in the same direction, yet few, other than manufacturers' sons, are taking much interest in the special technical schools described. The author found sons of English manufacturers in all parts of Germany picking up whatever knowledge they could. Thus one of these gentlemen was a chemist with one of the largest and most famous of Mülhausen dye-works, another was a kind of an apprentice in a München-Gladbach works where cotton blankets and other raised goods were manufactured, and many were being entertained by the colour manufacturers.

Turning to the United States, we find that schools of the class above described are springing up in most textile centres. The Fall River Textile School contains a small chemical laboratory and a dye-house containing vats for hank-dyeing, a machine for warp-dyeing, drying cylinders for warps, and other yarn-dyeing apparatus. The dyehouse is designed to supply the weaving department, and contains no piece-dyeing machines. The instructor thought that the schools of this class ought merely to supply their own needs, but admitted that such a procedure did not give scope for instruction in piece-dyeing, printing, etc., and therefore did not encourage research in these branches of the industry. Some people in the town of Fall River consider the school a valuable institution, whereas others laugh at it and say a lad can learn as much in the works in a month as he can learn in the school in a year. These facts serve to show that technical education has had to fight against a good deal of prejudice in America as well as in England, and that the American is not so eager for technical education as some people in this country are apt to think.

Lowell, Massachusetts, has a fine-looking school, which was built very capacious to allow for future demands. The author was informed that the intention of the school was to train engineers to become managers. All students were expected to go through the whole course; there were a few special students, but these are not encouraged, as a general all-round training in the engineering, electrical, 
chemical, and textile departments was aimed at. From the chemical department, which is fairly large, many chemists obtain positions in the local works. The department for woollen manufacture is particularly good ; some Yorkshire but mainly Massachusetts machines are installed. For cotton-dyeing, the equipment consists of two jiggers, a Klauder-Weldon dyeing machine, drying cylinders, and a few other machines. Trials were mostly done on an experimental scale: thus some mercerising trials were being performed at the time of the author's visit. Both here and at Fall River the machinery was driven electrically. As regards the staff of the school, one noticed that one dyeing instructor hailed from Manchester, the head of the weaving department from Preston, while most of the other professors and instructors were educated at the Massachusetts Institute of Technology or at the other technological institutions in America. Some of the people of Lowell look upon the school with favour, others with amused contempt.

The textile school at New Bedford is very similar to the one at Lowell. The school at Philadelphia, the oldest of its kind in the United States, also requires little further description. The dye-house at the latter school contains the usual small-scale apparatus for dyeing, yarn-dyeing becks, a hydro-extractor, a small warp-dyeing machine, and a small printing-machine. Professor Walker of the Massachusetts Institute of Technology describes industrial chemistry as the introduction of the dollar into the chemical equation, so that in his applied chemistry department he tries to bring the student into touch with chemical industry. Thus one finds the student separating pure from crude salt, and barium nitrate from common salt by means of a consideration of the respective solubility curves of these salts, making Glauber's salt, and afterwards designing a plant for its manufacture. An attempt is made to get the student to think, and the element of cost is introduced during the instruction. The principles of bleaching and dyeing are taught, and the lectures are supplemented by practical work. Special lectures, for example, on calico printing are given from time to time by men actually engaged in the industry. Very little 
instruction in dyeing is attempted. The director of applied chemistry said that the students from his charge never went to the textile schools, although he admitted that probably this was the best way for them to become of use. Yet he thought the chemist soon got into the work of the mill. Their idea was to train engineers, and the institute was really of the nature of a university. Their work overlapped that of Harvard University in the same city, but the University drew a different class of student. The number of their students had just been reduced because the standard of the entrance examination had been raised. At the primary schools, he thought, the scholars were taught obscure unnecessary subjects, and when they wish to enter for higher education they find that the entrance examination is too difficult: if they reduced the standard of this examination the duties of the students of the school were then too heavy. It is therefore evident that the same difficulty exists in America as has troubled our educationists for some considerable time.

At Columbia University, New York, a good feature of the chemical lectures is the inspection after the lecture of a large museum which contains many interesting objects connected with the subject which has been discussed. Visits to works are paid, and memoirs have to be written giving an account of the observations made. A little hankdyeing is done, being part of a practical course connected with lectures on industrial chemistry given to third and fourth year students.

As may be gathered from the author's remarks, he is of the opinion that the dyeing schools of other countries do not compare with those at, say, Manchester, Bradford, Glasgow, and Leeds, and even with many others of lesser importance in these islands. Just as we lead in the production, preparation, dyeing, bleaching, printing and finishing of textiles, so also is this lead maintained as far as the technical instruction applied to these industries is concerned. Also it must be said that there is little difference between the positions of England, Germany, and America as regards the appreciation which manufacturers show of the training received at these institutions.

But although England is foremost in this class of 
instruction, it must be patent to those knowing the conditions prevalent, that there are many respects in which our schools can be made more useful, and in this way gain more recognition from commercial men. The technical school should be the centre from which the manufacturers obtain the latest thoughts and progress in their industries. For instance, at the German colour-works, periodical conferences are held by the technical stafts, when any progress in colour manufacture is discussed and opinions on the products of rival works are given. In this way much valuable information is propagated. The publication of an abstract of these records would be exceedingly valuable to the dyeing world, and, although the issue of such a volume is improbable, one can conceive of such a publication being compiled by the stafts and students of our technical institutes. Manchester could report on innovations in the dyeing of cotton goods, and Bradford and Leeds on the treatment of wool. In this way much valuable information could be supplied to the industry, and such an action would meet with a response from the manufacturers. In connection with the recently formed Textile Institute there is the intention of supplying information to the trade, but surely much of this work ought to be performed by our textile schools. It is to be hoped, however, that this institute will be instrumental in acting as an intermediary between manufacture and the schools. Besides information concerning the properties of dye-stuffs, that concerning machinery might also be included in the report.

Again, our technical schools might advantageously adopt lectures on economics, e.g. banking, trade unionism, wages, organisation of industry and commerce, patent legislation, etc. Such lectures are given in some American institutions, and it is evident that instruction in the rudiments of these subjects, besides being interesting, would be extremely useful to those entering industry. Another point in which the writer thinks our schools are lacking is that concerning the prices of materials. The question of price should be held prominently before those evening students who are employed in works. We know that the uses of dye-stuffis are constantly being altered 
because of cheaper substitutes being introduced, and a consideration of the prices of these substitutes along with their other advantages and disadvantages is essential. It is true that the price of an artificial dye-stuff is a vague quantity, varying from district to district and depending on competition and other factors, but an idea of their approximate cost would be very useful to those students already engaged in or about to enter the industry. One finds in a works that such questions as cost of power, cost of damaged goods, and depreciation are factors which largely determine profits, whereas in the school one never gave such things consideration. Of course, one soon becomes aware of these losses after entering the works, but a slight previous knowledge of works conditions would be extremely useful. In the case of a student entering a dye-works direct from a university without previously passing through a technical school, the position is extremely awkward and discouraging. The employer is anxious that his profit should be increased, and expects to be taught something. The chemist is in a maze: he is paralysed by unusual circumstances, and sometimes is not allowed time to recover from his embarrassment. This period of embarrassment and consequent inactivity would have been much shorter if the chemist had previously attended a technical school. Such institutions exist to place him in touch with industry. Chemists are thinkers, and as industry thrives in consequence of the thoughts of those engaged in it, it is necessary that it should draw the greatest thinkers.

As regards the statement that some British manufacturers have little trust in technical education, it must be remembered that a large number of our manufacturers were originally working men, who by virtue of their ability and their thrift worked themselves into their present positions. ${ }^{1}$ These men do not understand what technical education really signifies, and therefore they cannot be expected to be very enthusiastic in its praise. But the danger arises when these manufacturers communicate

1 See Prof. S. J. Chapman and F. J. Marquis, "The Recruiting of the Employing Classes from the Ranks of the Wage Earners in the Cotton Industry," Journal of the Royal Statistical Society, vol. Ixv. pp. 293-313. 
their ideas to their sons, who in consequence are quite content with the education supplied by the local grammar school, and begin work under their father's care at the age of about fifteen years. It is true that this age is three or more years later than that at which the father made a start, but considering modern developments it is far too soon. What was sufficient for the father is by no means satisfactory for the son, if we are to train a new generation of scientific employers ; and every manufacturer should give his sons a chance of a scientific education and some technical training. It will be found that four or five years spent in such training before entering the works will yield ample returns, and will be far more valuable than many years of industrial experience, for it will be found that problems arising in the works will be far more easily grappled with. Each year of experience in the works is apt to follow a law of diminishing returns, in the sense that during the first few years much is learned, while in subsequent years fresh knowledge is acquired at a much less rapid rate. It is for this reason that works experience can be advantageously deferred until a system of mind training has been undergone. But it is impossible to obtain this mind training after one has entered the works, that is, when one is older; the scientific training must be obtained while young, and must follow the ordinary school training. 


\section{CHAPTER XII}

\section{COLOUR PRODUCTION ${ }^{1}$}

The German works-Past, present, and future-Combination and its possible effects-Our position-Possible remedies-Protection-Patent Legislation-Organisation of the industry in Germany.

THE German colour-works are perhaps the most perfectly developed and scientifically managed organisations in the world.

The English coal-tar colour industry has received much attention from many publicists, but our industry has principally been reviewed historically in order to elucidate our past faults. The past has been considered too much, whereas a peep into the future is of more importance. Everybody now knows the story of how Germany has deprived us of our aniline colour industry, how we send coal-tar to her and receive back finished products for which we pay dearly, and how difficult it is for us to compete with her in this industry now that her works are so large and so carefully organised. But the possibilities of development have not been argued.

The industry originated in England, and further progressed in this country as the result of a series of chemical triumphs. Hofmann was in England experimenting, but later, unfortunately for our industry, he returned to Germany. From this time we made little progress, because we had not the chemists to undertake the research work necessary for the industry. In Germany the conditions were different, for, thanks to Liebig, an

1 This chapter has been left practically as it appeared in the first edition, and has not been rewritten from the standpoint of the existing international crisis. The present situation is discussed in the next chapter. 
army of young chemists developed the industry in that country, relying on foreign raw materials. England was better prepared for the development of this industry because of our cheap acid, alkali, fuel, intermediate products, and raw materials, and because our gas industry, which supplied the crude coal-tar from which the dyestuffs were made, was more developed than that of any other country. Our position differed from that of Germany in that the latter country had the research chemists; we had not, or, rather, those that we had were either unwilling to take up this branch of work or were not encouraged to do so. At a later time the acid and alkali industries developed in Germany, and had to thank the aniline colour industry for this rapid change. The coaltar industry developed much more slowly than their dyestuff industry, and as a consequence Germany had to rely on foreign raw materials for the manufacture of dye-stuffs. The imports of raw materials for this purpose, however, became less and less, until at the present time the Germans practically supply all their own needs.

The success of their coal-tar colour industry is attributed by the Germans to their research chemists, but it should rather be stated that success was due to the encouragement of these chemists. At first the German chemists came to England for capital to work out their ideas, and many of them were engaged in the Manchester district; but they afterwards found that they could get the assistance they required at home, where such men as Bayer and Cassella, who were not themselves chemists, supplied the business and financial element. In short, research was encouraged in Germany but not in this country. Brilliant chemists left England to achieve great success in the German research laboratories. Professor $O$. N. Witt said progress in Germany had been due to the development of the people during the last hundred years, to research, to the combination of science and technique, and to the fortunate organisation of the chemical industry. Further, the Chemical Society and Industrial Chemical Society had had a big influence in fostering the industry through their journals.

That is the past, and the fact that these German 
colour-works now export about $£ 10,000,000$ worth of coal-tar colours and similar products, nearly a fifth of which are sent to this country, gives some idea of the present position of the industry in Germany.

In considering the loss of our industry we must not consider it a pure loss, but remember the people who would have been engaged in that industry are now following other pursuits. The actual loss is the difference in the income obtained in the two cases by the employment of the same labour and capital in different directions. Certain countries are better adapted than others for the working of certain industries, and the arrangement of industries between countries changes with time. Sometimes, indeed, the existence of an industry in a country is somewhat fortuitously determined. Thus the English cotton industry originally came to us from the Netherlands by the emigration of skilled workers from that country. This industry assumed a tremendous scale in this country, and in spite of the after developments of the industry in almost every other country of the world, we have maintained an unrivalled position in this branch of manufacture. It is seen that a small event decided the establishment of the industry in this country, and similarly we might say that a small event, namely, the encouragement of a few research chemists, decided the establishment of the aniline colour industry in Germany. But when we consider our position as regards the dye-stuff industry, we cannot for a moment maintain that our capital and labour is more economically directed at the present time than it would be if we were employed on a large scale in this industry; for we know that the German coal-tar dye-stuff industry is a very profitable one ; we know that we have great need of dye-stuffs; and we know that in the past the Germans have used our raw materials, and from the profits obtained by the chemical transformation of these materials have founded their industry on a sound basis.

The existence of this huge industry in Germany has also another effect. A short time ago a German professor deplored that Germany was not so progressive in the inorganic and analytical branches of chemistry as in organic chemistry. He said France and England were 
taking the lead in these branches. The fact is, that the colour-works in Germany are absorbing so many of the German chemical students, that as a consequence the organic side of chemistry is flourishing. In this instance we have the demand for labour influencing the direction of study, and the large colour-works have in this way been very influential in maintaining the standard of teaching of chemistry in Germany. Moreover, it is the author's firm belief that it is because of the development and the organisation of these colour-works that we hear so much of the scientific German, who is busy applying science to industry. The colour industry is a great asset to Germany.

Turning to the industry itself, we find in process of manufacture substances which were unknown a few years ago, and in some cases even a few months ago. With intellectual advancement man's tastes have become more and more complex, and in his taste for new colours lies the success of the industry under review. But, like most other innovations, artificial dye-stuffs were not at first enthusiastically received. After making some headway, there was a reaction in favour of the old methods of dyeing and printing, but finally the utility of the new products was proved, and the industry progressed at a great rate.

The separate firms have become very large and, moreover, there is a tendency towards combined working. Thus, there is a working understanding between the firms of F. Bayer of Elberfeld and Leverkusen, the Berlin ActienGesellschaft für Anilinfabrikation, and the Badische Anilin und Soda Fabrik. In fact, the Bayer and Badische firms already work their business together in the United States. Further, the two Frankfort works of L. Cassella of Mainkur, and Meister, Lucius \& Brüning of Hoechst, are acting in a similar manner, so that two large concerns promise to arise and to drive small competitors out of the dye-stuff market. That the small works are troublesome to the larger ones from time to time is shown by the following experience. A Swiss and a German firm were competing to supply a certain product, and the latter firm wished to come to an agreement as to the sharing of the profits, so as to avoid ruinous competition. The terms offered to the smaller Swiss firm, however, were not considered sufficient, 
and war began. The larger works probably won in the long-run, and also probably injured its competitor, for in this way the powerful combinations mentioned may get the entire market to themselves. Besides the combinations mentioned, it is also known that there are certain agreements between different firms of the two combinations. In fact, the whole German colour industry is organised so as to prevent ruinous competition, and intermediate products which are not required by one works are sold to others where they are required. At the time of the author's visit there was an agreement between two colour firms to keep up the price of sulphur blacks in München-Gladbach; the indigo convention between the Badische and Hoechst firms is also well known. Numerous other conventions exist in the colour industry, such as that between the British Alizarin Company and the foreign producers of that substance. Competition forces the prices of colours down enormously, and agreements like those mentioned above are welcome retreats for both parties. But if such overtures can take place between units of rival organisations, it is conceivable that a time will arrive when the German colour industry will be organised under one set of directors, or at any rate such a connection will exist between the combinations mentioned that a monopoly will be created. Such a powerful combination could undersell our small works, drive them from existence, and finally get the dyeing industry of this country entirely in its grasp. They might put the price up in America and at home, and, while keeping the price in this country very low, yet obtain a profit. Supply price may be placed below cost of production in order to gain a market. The price of an artificial colour is so uncertain a quantity that this "dumping" could easily be brought about if rigid combination existed in Germany. A German firm, which exploited the direct cotton colours, originally sold one of these colours at $6 \mathrm{~s}$. per lb., but nowadays the price obtained is only $6 \mathrm{~d}$. and the firm does not wish to supply it. This example gives one an idea of the profit obtained in the sales of these products, and shows that prices might in many cases be much reduced, in order to achieve some purpose, and yet a profit made.

In Germany there is a tendency for businesses to come 
to a common agreement. A kind of executive government of the heads of the various businesses is formed, and they agree upon a joint government ruling output and sales. They also fix the home price, and sometimes the "kartel," for such is the arrangement termed, pays a bounty on exports if there is any loss occasioned by being compelled to sell at a low price abroad. The loss is thus distributed among the whole of the businesses and does not cripple single concerns. The kartels differ from the present form of most of the American trusts in that they have not advanced to a unified management of production ; they are combinations for maintaining prices and, more or less, for the joint marketing of the produce by a number of concerns, which retain their own independence as producers. It must be said that combination often checks unhealthy competition and provides up-to-date conditions, and, if well worked and organised, a trust may be the very best of economic factors. Large-scale operations are, within limits, the most profitable and often result in better conditions for the workmen than in small works. In the United States the tendency is rather for the combination to become an individual business, a trust being formed by the sale of businesses to a company ; one huge business entirely absorbs the individual businesses. In Germany and in this country businesses show an inclination to get larger, but not to the same extent as in the United States. Moreover, in England the degree of trade combination is considerably less than in Germany and America, where this artificial state is aided by another artificial restriction, namely, the high tariff. The German kartels have notoriously sought to relieve the home market in time of depression by selling abroad below the domestic price.

Considering these facts, we come to understand our position as regards dye-stuffs. The dye-works of this country are so dependent on German dye-stuffs that it is not pleasant to contemplate what would happen if these supplies were stopped or interfered with. Any rupture in the trade between the two countries would lead to most serious difficulties, and our trade in dyed goods with other markets might be permanently and irretrievably injured. 
Prices are becoming so much cut that increases in cost of production, however slight, must be felt.

As regards the kartels existing in the colour industry, it must be remembered that although competition within the kartels is restricted, this arrangement sometimes gives a chance to the smaller works of obtaining business. Thus, in one instance, one of the big firms had had a contract for years and quoted for a renewal of the contract, having some idea of the price which the firms in the rival kartel could supply at, and knowing that the firms in its own kartel would not compete. The small outside producers were not, however, considered, with the result that the firm lost to one of the smaller firms a big contract. The small works are making a big fight, and are employing the best talent they can get, sometimes attracting men from the larger works. The larger organisations have technical men in all the industrial centres of the world, and these men meet from time to time at the German headquarters to discuss business matters. On one occasion one of the smaller colour firms sent men to stay at the same hotel as the other technical men mentioned, with the result that they heard all the doings of the conference discussed at table. This information they made use of, and now the rule has been made that the men must not discuss business matters off the premises. It is thus seen that the small firms are still full of life, and by their enterprise are preventing the consummation of a rigid monopoly in this industry.

Further combination will cause many dye-stuffs to be taken off the market, for the separate firms produce many of the same colours, although sometimes under different names. An individual concern, working in strict competition with all other concerns, had to supply as many colours as possible in order to satisfy their customers and in order in many cases to obtain fresh accounts. But with combination the tendency will be towards specialised production, and a series of the best colours will be produced. This arrangement will also assist the dyer in his selection.

Our position in the colour industry has often been pointed out and remedies suggested. Although largescale production has many advantages, it is seen that 
small works flourish in Germany and in Switzerland, and there is no reason why they should not do so in this country. Germany is a long way behind us in the textile industries, but she is gradually decreasing our lead. Although the larger the scale of production the more profitable production usually is, yet countries cannot be conceived as having large and unconquerable starts over other countries. One English firm is making headway on the Continent and is receiving respect from German chemists. Their dye-stuffs have received very favourable reports, while recent booklets issued from the works show no lack of enterprise. It must be remembered that one of the principal reasons why the German colour industry flourishes to-day is because of the application of keen business ability in the management.

Protection would have little effect in keeping out the German dye-stuffs, and the cost of production of our dyed goods would only thereby be raised. The German firms might erect works in this country, as they have done in France, Russia, and in other countries, and this arrangement might benefit employment a little, but the national benefit would only be small. Our present producers of coal-tar colours would not benefit materially, because they would not be allowed to work the German patents, so that our dyers would still have to import their colours or fall behind in the production of the latest shades; in order to obtain the novelties from time to time sent out by the colour firms, our dyers would have to pay the ordinary price, together with the tariff which had been placed on these products. In Germany many raw materials are admitted duty free, as coal, lime, tar, pitch, turpentine, wool and hair, raw copper, lead, tin, zinc, hides and skins, and some chemicals; but not alkali, alum, bleaching powder, or salt. In any case, considering the circumstances mentioned above, aniline colours are raw materials to our cotton, woollen, and other industries. In the United States salt and most chemicals are taxed; but white arsenic, sulphur, crude nitrates, petroleum, tar, turpentine, and wax are admitted free of duty. America, like Germany, recognises that the raw materials of industry should not be taxed. 
Another solution of the problem of aiding our almost defunct coal-tar colour industry, and one which deserves more attention, is that relating to our patent laws. These laws were intended to introduce new industries to the country, but by granting patents to foreigners we have prevented the development of many processes at home, and foreigners coming to this country have been granted monopolies which their own country refused them. It is generally conceded that making the inventor work his patent in the country where it is taken out-a method adopted by all European countries except our own-is the best direction for reform. The Germans worked our patents in their own country at the beginning of the coal-tar industry, and were so successful that they firmly established themselves financially. "English brains created the colour industry, English enterprise developed it, and English legislative folly has been the principal cause of its decline" (Ivan Levenstein, Nature, 1903). In the United States legislation has not been passed to force foreign inventors to work their patents in that country, but the high tariff has a somewhat similar effect. The question of patents, however, has to be carefully studied in order to ensure against evasion of the compulsory working clauses, for there is the possibility of an inventor working his patent on a very small scale, thus satisfying the clauses mentioned, but at the same time preserving his monopoly. Legislation regarding patents is of great importance, and some German technical schools have lecture courses on this subject.

Turning to particular examples of patented products, we find the German firms, immediately they discover a new colour, patenting it all over the world. Of course the chemists turn out many colours which, after consideration, are not put on the market, but those which are considered worthy of manufacture are well guarded and patented. One German firm employs for this purpose a man who holds doctor's degrees both in chemistry and in law. The German firms are continually taking out new patents, and if this rate of progress continues one can conceive of a time when the chemical part of the colour industry will be so far developed that little further progress can be 
made. Then after a term of years all patents will have run out, and other manufacturers will be able to make the previously protected products. The Germans recognise the possibility of this development and, while continually bringing out new products, they make their profits out of them while they are new; the prices obtained for real novelties are high, but after a time they sink considerably. Moreover, the German colour-works, recognising the probability of other manufacturers producing their colours when their patent rights run out, are taking great care that their specifications should be designed to give protection, but at the same time little information as regards the working of the patents. In this matter they have shown great cleverness. Further, they are spending large sums on research work, because they recognise that only in this way can they flourish.

England has set examples to the world and has been imitated on all hands in many business matters, and has so created many competitors. As regards our position in the colour industry, it is evident that we can only achieve success by following the methods by means of which the Germans have succeeded.

Of the German colour-works the Badische Anilin und Soda Fabrik was founded in 1865, at which time the work-people numbered 30. In 1900 they employed 6200 hands and had a capital of 21 million marks. They have works in Germany, France, and Russia. In 1900 they had 148 chemists, 75 engineers, and 305 clerks at the German works.

The development of the Bayer firm is interesting as showing the change from the sale of natural colouring matters, as indigo and safflower, to the manufacture of magenta and its congeners in 1860, and then to the production of alizarine and its derivatives in 1870. Later, they introduced their benzo-purpurines and delta-purpurines, which were the first substantive cotton colours, while shortly afterwards they brought out a whole series of this class of colours. Next came diazo blacks and other diazo colours, para red, diamond blacks, alizarine cyanines, and brilliant alizarine blue. This firm also introduced the copper sulphate treatment of dyed goods to increase the 
fastness of the colours. They were not far behind when sulphur colours were introduced, and they produced the first green. The Bayer firm has advanced very rapidly, until now it is one of the most important. It is significant in this connection to note that they have the most patents, 1000 German and 1200 foreign, and at the time of the author's visit employed the most chemists.

The business side of the German colour-works is highly developed. They make all they possibly can for themselves. Passing through the departments in which the different handbooks are made, one is struck with the enormous trouble and expense of such productions. Many of these are quite artistically produced, and contain scores of patterns, all of which have to be fixed by hand. These patterns are issued for cotton, wool, half-wool, silk, halfsilk, leather, soap tints, straw, buttons, wood plait for hats, wood stains for picture frames, etc., and the intending customer is thus presented with reliable and trustworthy samples of the dyed article besides elaborate instructions how to perform the dyeing. Also some of the handbooks give drawings of the latest machinery in use, and although this is of German manufacture, yet one gets an idea of what is being done in Germany. The books are issued in about a dozen languages, including Japanese. The importance of good pattern-books is manifest in many industries, and the Germans seem to have developed this branch of business to a high state. Hundreds of clerks are seen at work in the different factories, and from the scores employed in the English departments, occupied solely with the trade with England, one gets an idea of the magnitude of that trade.

In all cases the works are built where good transport facilities exist. Thus, most of them are situated on or near the Rhine, while the Berlin Company's works is on the Spree and thus in connection with Hamburg. All have ample railway communication, and in some cases private lines. As one stands on the banks of the Rhine and watches the many barges floating down the stream, one is reminded that this river is a mighty factor in German commerce. Here we have no large initial capital expenditure as in the case of our canals, but a natural 
means of the cheapest transport; the products of the industries of Rhineland are thus taken down to the sea and enabled to compete where otherwise they would not have a chance. The importance of cheap transport is becoming greater and greater, and it has often been said that England, both as regards external and internal communication, is the home of transport anomaly. The way that some foreign goods are carried to parts of England at cheaper rates than our own produce, and the way that English ships carry goods from other countries a greater distance at a cheaper rate than they carry ours, thus defeating us in some foreign markets, has often been mentioned and deplored. However, it must be remembered that the enormous development of Rhineland as an industrial district had resulted largely from the presence of the Rhine and its use as a means of transport. Moreover, this fact should not be lost sight of in comparing the relative growth of England and Germany industrially. It is said that the Thames has done more for England than all its canals and perhaps even its railroads.

An interesting part of the colour-works is the manufacture of the dye-stuffs themselves. Of course different dye-stuffs require different methods of production. In one case a building about 70 feet high was used. This had big tanks arranged at different heights and containing different chemical preparations. The liquids from these tanks were run at certain periods into a vat, and the dye-stuff was produced by interaction. It settled out of solution on standing, and by filtering through presses came out like clay. The liquid filtered off is further utilised, and the clay-like mass is put into barrels and taken to the drying ovens. These ovens are heated by fires below the ground, and the dye-stuff is wheeled into the ovens at the ground level. Different temperatures are used for drying, according to the nature of the dye-stuff being dried, and the ovens are specially constructed to admit of regulation. The dye-stuff is dug out of the barrels by means of spades and placed on trays, for transference to the ovens. Dyestuffs of all kinds are scattered about the ground, giving it a peculiar appearance on a wet day. Moreover, one sees workmen of all colours emerging from their working 
quarters and making for the baths. Each labourer engaged in dirty work has to have a bath before leaving the works, and they require it.

A large amount of ice is used in the colour-works for cooling purposes during the manufacture of diazo colours, and this ice is made on the premises. At one works, long rectangular iron boxes were seen standing vertically in brine, which is maintained at a low temperature. These boxes are filled with water and agitated by a lever arrangement; when the water is frozen the iron boxes are tilted and the ice removed. Some works make as much as 200 tons of ice daily.

At Bayer's Leverkusen Works, on the Rhine, near Cologne, the water-supply is obtained from a deep well. The pumping-engines are capable of raising 60,000 cubic metres of water per day, and this is 10,000 cubic metres in excess of the daily consumption of the city of Cologne. Of course this amount of water is not used at present, but the whole of this works is designed for future needs. There is plenty of room for expansion.

The author paid a visit to a works at Nüremberg where colours of a different class were made. Nüremberg has many industries, and prominent among these are the production of mirrors and of lithographic supplies. At the works mentioned fine metallic colours were produced by heating powdered metal with aniline colours. Different alloys of copper and zinc are used. Aluminium is used alone for this purpose, but not along with the other two metals, as a slight amount of aluminium produces brittleness and spoils the whole melt. Aluminium had at the time of the author's visit risen greatly in price, owing to an understanding between European and American producers. The copper used is the purest metal obtainable, and is usually produced electrolytically; it is beaten out with hammers and washed with sulphuric and tartaric acids. The colours are used for wall-papers, print cloths, and in lithography. The trade with Great Britain is large.

In recent years marked advances have been made by English employers as regards the housing of their employees, and similarly in Germany much has been done in this direction. This is particularly the case at the large 
German colour-works. Thus, at Hoechst there is quite a town of artisan dwellings, each of which has a garden. Also there is a store, where provisions are sold by the firm at little more than cost price, a school, a continuation school for workmen, a library, an assembly hall, a huge dining-hall, and other conveniences. Hoechst itself is a very dirty place, but by the aid of a policeman and the increasing intensity of the fumes proceeding from one of the works' chimneys, the author was able to find his way through the unpleasant portions of the town to the much better district, where the colour firm's work-people were housed. The neat dwellings in this quarter are in big contrast with the squalid shanties of the town itself. But it must be mentioned that it is only the more respectable work-people who live in the houses mentioned; the lowest classes find other places of abode. At all the works good kitchens provide excellent, cheap dinners. Besides the above conveniences some works have also savings-banks, hospitals, long-service premiums, pension funds, workmen's relief funds, musical societies, clubs, etc. A director of one concern sends workmen's wives and little ones to summer resorts, and pays for milk for the children at certain times of the year. The German firms are making huge profits, and some of these returns they are investing in a profitable and productive manner, that is, in caring for those who labour to make the profits.

At Mülhausen there is a workmen's colony which was founded in 1850 to improve the conditions of the workmen of the town. It consists of about 1000 houses with small gardens, which were sold to artisans at about cost price. The district at the present time, however, is hardly in the best condition, and many of the houses are untenanted, because it is said the workmen are anxious to live outside the town, and to travel into town in the morning by train. There are baths, wash-houses, and schools in the colony, but at the time of the author's visit the whole appeared to be a mixture of disorder and small businesses, only a part of the houses being used as originally intended.

Returning to the German colour-works, we find in the technical departments chemists engaged as analysts, as research chemists, and as technical men. The first class of 
chemists deal with the work materials and products ; they also analyse the products of other firms and attempt to detect infringements of patents. The research chemists are under the direction of distinguished chemists as Duisberg and Bernthsen. A list of the chemists who have been employed from time to time by these works shows that the best brains have been attracted, and the same conclusion is formed as to the present state of affairs when one surveys the list of distinguished chemists who came from Germany to England for Sir W. H. Perkin's coal-tar jubilee.

The technical men are concerned with the dyeing properties of the different dye-stuffs produced. In this department all their own products and the products of other firms are tested. As soon as a dye-stuff is put on the market by a rival firm it is first analysed and then investigated as regards its dyeing properties by one of the technical staff. A conference is called, at which the technical man reports on the characteristics of the new colour, and suggests one or more of their own colours or mixtures of colours which can be used in competition with the new article. The travellers are then provided with this information in order to compete with the new product ; if one of their customers mentions the advantages of the new product, the travellers are ready with the information to convince him that by mixing their - BX with a little -BR, he can get the same result at less cost.

The chemists who investigate the dye-stuffs as above mentioned are generally university graduates, who, on entering the works, are trained to become technologists or to go out as representatives of the firm. Some of these men are sent to all parts of the world as special travellers, while others attain positions as superintendents of the different departments. Most of the heads of departments and many occupying high positions in the actual manufacture of the colours, have gone direct from the university into the works, and the advantage of employing university men is well shown. No industry employs more university graduates, and no industry is more prosperous. These university men gradually rise to occupy the highest positions. A "good practical man," as the saying is in 
England, might discharge the duties very well, but as a rule he is not imaginative, and he cannot suggest improvements or keep in touch with progress through the technical journals.

University graduates are received for training as technical men at a salary of about $£ 100$ a year, and a contract is made for one year. If during that year satisfaction is given, their salary is gradually raised during a period of five years, for each year of which a separate contract is entered into. Most of the servants of these works are under similar contracts. If any breach takes place between employer and employed, the latter is not allowed to enter the employment of a rival organisation during a period of two years after the breaking of the connection, but during this time he receives his salary from the firm. In this way a man, who has gained some special information in a department, cannot immediately place that information at the disposal of a rival firm; and after the two years agreed to, the information, owing to the rapid progress of the industry, may be of little use. During the first year or more the men engaged as above mentioned merely collect information, and are of little direct use to the firm employing them. It is true that they have to report on various products as before stated, but most of their time is taken up in gathering as much practical knowledge as they can in order to fit themselves for subsequent duties. They are for the most part left to themselves; they use the splendid libraries which many of these works contain, experiment as they wish, and learn as much as possible. In fact, this training is somewhat similar to the instruction provided by technical schools. The men meet periodically, when anything new in the chemical world is discussed, and the technical papers are passed round. In this way all are kept in touch with progress in the industry.

The actual dyeing is performed in a laboratory containing some dyeing machinery, which is worked by labourers to the directions of the technical men. Some modern machinery is used, but it is more or less on an experimental scale, and simply used to test the different dye-stuffs. 
In fact, the whole training of these technical men is designed because of the lack in Germany of good dyeing schools. A graduate of a university rarely enters the trade schools, so that the colour-works have to provide the training themselves. But in England, where we have excellent schools of dyeing, these schools could be used in place of the experimental departments named, and a student who has gone through a training in dyeing at a good technical school would require little further instruction before being called upon to discharge the duties for which the German technical men are trained. The author speaks from experience when he says that a university graduate can learn as much by a year at an English technical school, as he could by a year's employment in a technical department of a German colour-works as described. Indeed, if it is contemplated that technical men be employed in connection with the new British dye scheme, the right men to engage would be those with university training, together with some technical school or industrial experience.

After the technical men have been engaged a certain time at the duties mentioned, and when the directors think that these men are sufficiently trained, they are sent into a works in order to obtain some industrial experience, that is, if they have not previously obtained this experience. If a man is intended for travelling in England, he is sent to an English works which is on good terms with the colour-works, and in this way he learns in about six weeks' time a little about the organisation, equipment, and occupation of the works, which some day he may be called upon to visit for business purposes. On completion of this training the technical man returns to the experimental laboratory, and after a short time he is ready to carry out the work for which he has been trained. Some of these men travel in districts near the works, returning perhaps weekly, while others go abroad. All the travellers visit the works from time to time, but in the case of those working, for instance, in America, the visits are of course not frequent. These men are also sent out when technical difficulties are experienced at the different works with which the colour firms do business. For instance, at a 
certain print-works the chemist died, and it was found impossible to carry on the business without him. During his lifetime he had kept secret all his recipes, and he had different signs for different materials, so that when he suddenly died there was no means of finding out in what way and with what materials he had made the various " mixings" used by the firm. The works was at a standstill, so they applied to one of the colour-works for assistance. One of their technical men was sent, and after three weeks' hard work all was in order again. But there was one difference between the new circumstances and those previously prevailing. The chemist in making up the new recipes had used only the colours of the firm who sent him, so that by this means the trade in dye-stuffs and some other materials was diverted to his firm.

A large staff of research chemists is employed at each of the works. Their remuneration is only moderate, but successful work leads to increased earnings; patents are taken out by the firm, and if successful the inventor gets a share of the profits. In this way many chemists are drawing large amounts, and this system certainly incites the men to their best efforts. The preparation of a new dye-stuff is usually entered into after the constitution of that dye-stuff has been found, just as the formula for indigo was discovered before it was prepared on the large scale, and the whole series of magenta colours were produced by introducing groups of various kinds into the triphenylmethane nucleus; but in the case of sulphur colours, whose constitutions are not definitely known, new colours of this series are not produced with the same certainty.

The different colour firms aim at producing a variety of products, because business obtained for one product may lead to other business. For this reason, the travellers of some colour firms have been known to urge their employers to either make indigo or to sell the natural variety in order to satisfy all classes of customers.

In the works, each man is confined to his own department, and is in no case allowed to visit other departments. Men occupying high positions are known to have never been in any other part of the works than their own small building. There is a case on record of a chemist engaged 
at a school who used to speak against the products of one colour firm. This firm gave him a good position in order to keep him quiet.

As regards the experimental dye-houses of the different colour-works that at Hoechst (Meister, Lucius \& Brüning) is about 30 yards square, and is fitted with small machinery, pots, and other requisites for dye trials. Round the sides of this laboratory, and connected with it, are smaller laboratories, which are devoted to special purposes, such as cotton, wool, and silk dyeing, paper dyeing, indigo dyeing, etc. A large number of chemists are employed, and here, as at other German works, Englishmen were seen. In the indigo room the familiar spiderweb-like hexagonal frames were used for dyeing piecegoods. Volunteers (see pp. 87 and 114) also work in this department, and they have good opportunities for learning. Just previous to the author's visit to this works, one of the technical men had invented a machine for dyeing cotton cloth a series of colours which blend into one another. The machine consists of a pair of rollers, between which the cloth passes ; the lower roller, instead of being in one piece, is divided into numerous sections, the sizes of which vary, and each section fits into a separate small trough. The machine is like the ordinary padding machine, except that the box and the lower roller are in sections. Different dye solutions are placed in the different sections of the trough, and in this way the cloth is dyed differently at different parts. If different strengths of the same dye liquor be used, beautiful blendings are produced, and the variety and perfection of the effect depends on the number of sections in the trough. Another box, exactly corresponding to the trough used, is employed for adding the dye liquors to the trough. Many varieties of the machine are at work, and it is made by Haubold's of Chemnitz.

In Cassella's laboratory they have cop-dyeing and other machinery. A French modification of the Mather and Platt steamer is used. Cassella's printing trade is growing rapidly, and consequently their experimental printing department shows the same change. Their experimental laboratories appear to be well conducted, but they are old. In the different machines some patterns are dyed, but 
most of these are obtained from manufacturers using their dye-stuffs. The youths who labour for the technical men begin the work while very young, and after a time attain such skill that they can carry out the dye trials without direction. Their wages are very small, as the work is quite mechanical. The weighing out of the dye-stuffs is done in a separate room, usually by girls. Some youths are engaged entirely in testing the fastness of dye-stuffs to washing, reagents, etc. Manufacturers send a pattern with instructions that the dye-stuff required must be as fast as that used for the pattern ; this request necessitates tests for fastness, and it is a good principle that this firm never relies on any previous trials. In testing the strength of a dye-stuff and comparing it with a pattern, all the dyestuff is salted out of solution on to the material, and afterwards another piece of material is put into the bath to show whether it is exhausted; this second piece of material is attached to the first piece when making a judgment of dye-stuff strength.

In the laboratory of the Badische works there is a machine in which cop-dyeing is done by frothing, a Pornitz cop-dyeing machine, and an Obermaier machine for treating loose fibres. Felting tests were done by working the material along with soap between two wooden slabs. In testing the strength of dye-stuffs used for dyeing paper pulp, a certain amount of pulp is mixed with a solution of a certain amount of the dye-stuff and stirred by means of a vertical stirrer. The pulp is then filtered by pressure, and the paper thus formed is first dried between absorbent paper and then in a steam bath before comparing the strength of the dyeing thus obtained. In the leather-dyeing department skins were shaken with solvents to extract the fatty matter, and then dyed back to back to prevent the flesh side from absorbing the dyestuff. Sometimes the skins were dyed by merely painting them with a solution of the dye-stuff. Leather was also seen in process of dyeing, and afterwards polished in a machine provided with a small smooth roller, which revolved against the surface of the leather.

At the Berlin Company's works they have a neat milling machine, the material being moved backwards, 


\section{DYEING IN GERMANY AND AMERICA}

forwards, and sideways during the process by means of rods operated by different eccentrics. The walls of one room were covered with small drawers, which contained the products of competitors ; each firm finds it necessary to learn as much as possible concerning the productions of its competitors.

The large German works contain departments in which men are taught to dye their products in all possible ways. These departments are called "volunteer laboratories," and those receiving instruction are either sons of manufacturers, or in some other way connected with, or about to enter, the dyeing industry. This scheme is advantageous to both parties concerned. In the first place, the firm profits because the volunteer learns to dye his colours thoroughly; and, since he is under contract not to volunteer with another works for one year, he afterwards uses in practice the colours he has learned so much about. Then the student gains in general knowledge, for he learns to dye materials which, perhaps, he has not seen before. Thus, he can learn the dyeing on the small scale of cotton, wool, half-wool, wool and mercerised cotton, silk, half-silk, jute, linen, ramie, artificial silk, shoddy, etc. $\mathrm{He}$ is placed under a competent chemist, and, as he also has a fine library at his disposal, he has every opportunity of acquiring knowledge. He also has the opportunity of keeping in touch with the scientific journals, for in this way one learns the latest discoveries in technology. In some works the volunteers move freely in the technical laboratories, but in others they are kept to their own department. From time to time discoveries, which have been made as regards dyeing, are tested in these laboratories, and men pay periodic visits from different works in order to keep in touch with progress in their industry. By means of pattern-books and note-books the volunteers keep records of their work, and these notes subsequently prove useful in industry. Moreover, by meeting young dyers of different nationalities in these departments, one gets an idea of the nature of the dyeing industry in different parts of Europe. 


\section{CHAPTER XIII}

\section{COLOUR PRODUCTION (continued)}

IN the previous chapter, which has been left practically as it appeared in the first edition, some aspects of the colour industry have been discussed. For instance, the effect of war between this country and Germany was alluded to, and the chaotic state which would thereby result. As regards the compulsory working of patents we have recently had legislation on this matter, but it is evident that the new Act has been so evaded as to make it practically useless. The author mentioned this possibility, and the outbreak of war has revealed the methods used by the Germans to evade the law. In the United States compulsory working is not demanded of patents, because they regard such legislation as incapable of achieving its purpose. Then in reference to the production of new colouring matters the author pointed to the possible exhaustion of the field, and it is significant, in looking back over the last ten years, that relatively few discoveries of an important nature have been made in coal-tar colour chemistry. The discovery of Thio-Indigo Red, before the decade mentioned, led to the production of other vat colours, such as, Thio-Indigo Scarlet, the Algol and Ciba colours, and then many new brands of older classes were introduced; but the range of colours has not been very much extended. In fact, dyers who use no colours which have been discovered during the last ten years may be prominent in their industry. Indeed, in this country, many large works still use wood colours in large quantities, particularly for yarn, and they are not inclined to give up their use. It can therefore be understood that, as the patent rights of the important aniline colours run out, the 
aniline dye industry will assume another aspect. As previously mentioned, the Germans have recognised this possibility, and have disguised their patent specifications so as to give little information as to the method of production of the patented articles. Moreover, they have turned their attention more and more towards the production of other synthetic products, as photographic and pharmaceutical articles. After a long run, and when this branch is played out, they will no doubt find some other branch of remunerative activity.

The German aniline colour industry has been so much discussed in this country of recent years, and at present is receiving so much attention, that one is apt to lose all sense of proportion in regarding it. It must, however, be remembered that this industry is only a relatively small part of the total chemical industry of any country. Indeed, if the chemical industry as a whole be considered, it will be found that, as regards the value of chemicals produced per head of the population, Great Britain is not far, if at all, behind Germany. On this point the author wrote the following remarks in a letter to the Manchester Guardian in 1910: "As for the German colour industry I have long contended that it has too often provided food for those Britishers who are always stating that we are falling behind in the industrial race, and the fact that this industry is again brought to our notice only serves to point to the paucity of more suitable material to brood over. Even though the dividends declared by the German colour-works are high, it must be remembered that there are still many English chemical concerns which are the envy of German manufacturers. For instance, to-day I received a German paper that contained an advertisement for a chemical commodity which is made not far from Manchester. This article is made by a firm whose enterprise is world famous, whose capital is as big, I think, as all the German colour-works put together, and whose profit in normal times is very large. This is merely one concern in one British industry, but such facts are often overlooked when generalisations are made."

Although Germany is foremost as regards the production of synthetic dyes and some fine chemicals, it must 
be remembered that our heavy chemical industry is of great importance, and that we also produce large quantities of fine chemicals of prime importance. Thus, as regards alkaloids, Germany was our principal customer before the war. Then, more morphia is produced in this country than we require for our needs, and caffeine is made to an enormous extent. Chloroform is produced in large quantities and exported to all parts of the world. As regards synthetic drugs, it is true Germany has produced some articles of value, but at one time hardly a month passed without some new drug with a scientific name being introduced to English medicine. The new products differed only slightly from those previously used, and few were of value; they only served to cause annoyance to medical men. Moreover, the few of these drugs that are of value can be made in this country without much difficulty. It has also been pointed out that some drugs which were discovered in this country have been given high-sounding names by the Germans, and by this means the trade in these articles has been diverted to themselves.

It is recognised that, except as regards synthetic dyestuffs, synthetic drugs, and fine chemicals, the English chemical industry has held its own ; but it is also manifest that the Germans are now turning their attention to the capture of the heavy chemical trade, for their exports of sulphuric acid, cyanides, etc., are increasing.

There is no doubt, however, that the German aniline colour industry, as explained in the previous chapter, has been a great industrial asset to Germany, because it has become the mother of many new industries, such as synthetic pharmaceutical products, anhydrosulphuric acid, and liquid chlorine; because the industry has been a great object-lesson to the Germans in showing the advantage derived from the application of science to industry ; because it has reacted in producing a more general appreciation of technical education; and because it has been an important factor in inducing the Government and local authorities to render assistance in fostering various industries. Co-operation among factories in Germany enables different syndicates to obtain facilities from their Government which we cannot obtain here, and this lack 


\section{DYEING IN GERMANY AND AMERICA}

of cohesion among our chemical manufacturers creates a tendency to compete unduly with one another, whereas some system of co-operation and control, such as they have in Germany, would be of value both in cheapening working costs and in regularising output.

In 1913 the number of coal-tar dye-works in the world was: Germany 22, France 11, Great Britain 11, United States 9, Austria-Hungary 4, Switzerland 4, Holland 2, Russia 2, Belgium 1, Greece 1, and Italy 1. Sixteen plants, which at one time made coal-tar colours, have abandoned the manufacture: 11 in Germany and one each in Austria, Belgium, France, Great Britain, and Switzerland. Fourteen have been absorbed by other factories: six in Germany, four in Switzerland, two in France, and one each in Holland and Belgium. These figures do not give an idea of the relative importance of the different countries in the dye-stuff industry, but the following returns give some idea of the eminence of Germany's trade. The values of coal-tar dye-stuffs imported into the United Kingdom, India, and Japan from Germany in 1913 were :-

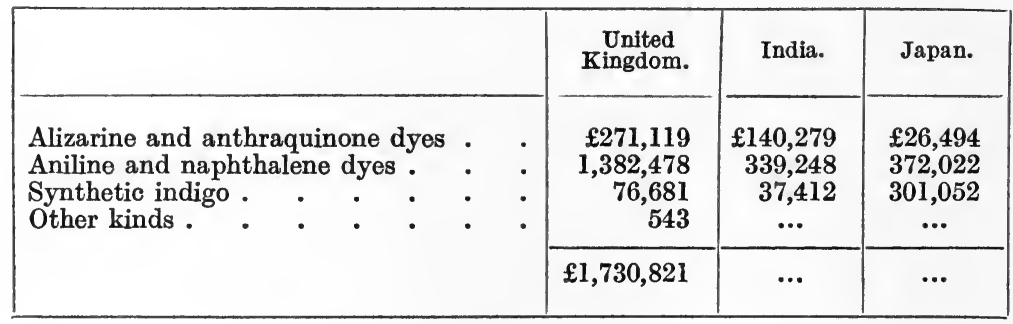

China imported synthetic indigo from Germany of the value of $£ 575,000$.

On the outbreak of war the stoppage of imports from Germany placed users of dye-stuffs in an anxious position. Substitutes were found in some cases, and it was remarkable to find how often other colours and pigments could be used in place of the usual dye-stuffs. Wood colours were more used, and the Swiss firms assisted matters considerably; but at times such colours as basic blues, basic violets, and acid violets were practically unobtain- 
German Exports of Tar Products and Dye-Stuffs in 1913 (GIVEN IN Metric TONS)

\begin{tabular}{|c|c|c|c|c|c|c|c|c|}
\hline Country. & 晋。 & 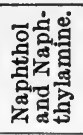 & 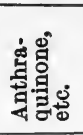 & 晋通 & 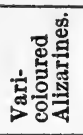 & 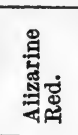 & 总 & 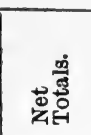 \\
\hline $\begin{array}{l}\text { United States } \\
\text { Great Britain } \\
\text { Austria }\end{array}$ & $\begin{array}{r}2,428 \\
340 \\
655 \\
650 \\
602 \\
120 \\
1,217\end{array}$ & $\begin{array}{c}638 \\
333 \\
109 \\
\dddot{11} \\
\dddot{117} \\
\dddot{217} \\
272\end{array}$ & $\begin{array}{r}965 \\
34 \\
284 \\
825 \\
998 \\
108 \\
776 \\
1,201\end{array}$ & $\begin{array}{r}13,855 \\
10,793 \\
5,582 \\
4,097 \\
1,098 \\
2,700 \\
1,301 \\
941\end{array}$ & $\begin{array}{r}2,164 \\
1,130 \\
223 \\
158 \\
156 \\
96 \\
124 \\
\cdots\end{array}$ & $\begin{array}{r}493 \\
1,493 \\
207 \\
\dddot{17} \\
\cdots \\
98 \\
259\end{array}$ & $\begin{array}{r}3,461 \\
1,180 \\
1,361 \\
662 \\
434 \\
313 \\
323 \\
\cdots\end{array}$ & $\begin{array}{r}24,004 \\
15,235 \\
7,975 \\
6,392 \\
4,579 \\
3,037 \\
2,839 \\
2,008\end{array}$ \\
\hline Net total & 6,012 & 2,686 & 5,123 & 38,185 & 3,605 & 2,724 & 7,734 & 66,069 \\
\hline $\begin{array}{l}\text { Number of countries } \\
\text { Germany exported } \\
\text { to }\end{array}$ & 11 & 6 & 11 & 32 & 12 & 9 & 21 & $\cdots$ \\
\hline
\end{tabular}

able. Yet, to those in the trade it has been really wonderful how the reasonable requirements of the public have been met in the textile trades during the unforeseen circumstances arising out of the war. Those engaged in business have, of course, been reticent as to their methods of procedure and their means of overcoming the difficulties which have arisen. Publicity as to these matters would have served no good purpose, and might even have been harmful from a national point of view.

When the war broke out it was evident that the stoppage of the imports of aniline dye-stuffs from Germany into this country would have a big effect on a number of our industries, and the matter engaged the attention of the Government. The President of the Board of Trade appointed a Committee to investigate the matter, and an Intelligence Branch was opened to bring together buyers and sellers of articles hitherto imported from enemy countries. Acts were passed to extend the powers of the Board of Trade as regards patents during the continuance of hostilities. The exportation of dye-stuffs from this country was prohibited by decree dated September 8, 1914, but a further Order of Council granted licences to certain firms to export certain dye-stuffs. On September 15 the German Government also issued a decree prohibiting the 
export of dye-stuffs and tar products, but it is known that since that time both intermediate products and dye-stuffs have been exported to Switzerland and thence to other countries. In fact, the Germans later threatened to supply the Swiss with no further products if these were exported to England.

In November 1914 the Board of Trade, after consultation with the Committee appointed, deemed it advisable to take such steps as were possible to develop the immediately available sources of supply, and also to encourage the permanent manufacture of dye-stuffs in the United Kingdom on the large scale, so as to guard against any recurrence of the present difficulty. Arrangements were made to encourage the immediate expansion of the various existing sources of supply, and, as regards the permanent supply, after consulting representatives of various colour-using industries, a scheme for the formation of a limited company was devised, the bulk of the capital of this company to be subscribed by the consumers of dye-stuffs and others interested. The Government indicated their willingness to subscribe a certain proportion of the share capital, and to guarantee the interest on a large debenture issue for a term of years. It was pointed out that the trouble was not a temporary one, for after the war the two large German combines could so dominate our great textile industry that they could overcharge and hamper it pending the time when they could challenge its very existence. The share capital of the new company was stated at $£ 3,000,000$ divided into $3,000,000$ shares of $£ 1$ each, the Government to advance to such company $£ 1,500,000$ bearing interest at the rate of 4 per cent per annum and secured as a first charge on the assets and undertaking of the company, and to be repayable in twenty-five years. Arrangements were made with Read, Holliday \& Sons, Ltd., for the extension of their plant, and later, this works was taken over by the new company. The Government stated that they should have the right of appointing two directors, and that they considered co-operation could best be secured by the subscription of the share capital by those interested, and also by a contract being entered into between the company and the con- 
sumers and users of the products, whereby the consumer should agree for a period of five years after peace is established to take supplies from the company in all cases where the company is able to supply the same of good quality and at reasonable prices, but with the provision that, if the consumer thinks that the prices charged by the company are too high, he may require that the prices charged shall be determined by an independent referee. With the object of securing for the company impartial administration, the Board was chosen by the Committee, and was mainly composed of business men who were not themselves necessarily engaged in the dyeing trade. An Advisory Committee was formed, mainly from representatives of users of dye-stuffs, and in addition the Board had the advantage of the advice of chemical and other experts.

The scheme met with much opposition. For instance, the Leeds Chamber of Commerce suggested protection for the new industry by means of import duties, and, later, stated that the Government scheme would not meet the needs of the situation, but would stifle competition and establish a monopoly; the Council of the Associated Chambers of Commerce supported the Leeds Chamber; the London Chamber of Commerce asked for protection for ten years after the conclusion of peace ; the Manchester Chamber of Commerce expressed itself against protection for the new industry ; and the Derby Chamber of Commerce preferred private enterprise to a Government undertaking, because small undertakings to manufacture at first quite a limited number of colours would, they thought, be more successful than one large concern. They further stated that the movement would be assisted by co-operation between gas works, tar distillers, alkali works, etc., for the purpose of advancing the crude products another stage. Objections were also raised in other quarters. Many firms hesitated to bind themselves under the contracting clause, which compelled them to buy only from the new company, owing to their doubts as to the ability of the company to produce dyes of the requisite quality and at a suitable price. Moreover, the subordination of science to business was considered by some people as a weakness of the scheme, since it was thought that science 
should govern the directorate. Resignations from the Board took place because of differences, but during this criticism it was pointed out (1) that the Government only lent their assistance because private enterprise had not taken the matter up, and (2) the co-operation of the users of dye-stuffs was required because, if the users and the allied trades resolved that never again would they be placed in such jeopardy, the Germans would be powerless and the British company unassailable.

Opposition, it was said, came principally from the three largest dyeing concerns in the country, and it was stated that these works could look after themselves, whereas the smaller works could not. The success of the scheme depended largely on the manner in which the German patents were administered, and the Government stated that it was their intention not to cripple the company after the war was over, but to give it every opportunity of making the most of those patents. The Government left open for discussion the payment of royalties in respect of these patents, but it was stated that the operation of the patents undertaken by the new company would be allowed to proceed after the war was over without interruption or hindrance. The grant of $£ 100,000$ towards research was stated to be really a grant towards assisting technical education. Against the criticism that no chemist was on the Board, it was urged that many chemical concerns had been ruined by having highly gifted scientific men on their Boards.

The result of the criticism was that the Committee was altered and a modified scheme introduced. The financial basis of the company was also altered, and the agreement to be entered into between users of dyes and the company was considerably modified in favour of the users. On March 27, 1915, sufficient applications for shares had been obtained to enable the Committee to decide to go on with the company. A research department was afterwards formed, and a Technical Committee and a strong Advisory Council nominated. It was stated that the output of Read, Holliday \& Sons works had been doubled since the taking over by the company, and further plant was in course of erection. It was essential to increase largely 
the capacity of the plant for the manufacture of intermediate products, and to this end a new works was established near Huddersfield.

In America, at the outbreak of war, the position was very similar to that in this country. They had also been dependent on Germany for supplies of dye-stuffs, and with the stoppage of imports they, like ourselves, had to seek other means of obtaining these products. The authorities at $\mathrm{W}$ ashington tried to make arrangements for importing dye-stuffs from Germany, but without success. It was pointed out that other countries would not require the stocks of dye-stuffs held in Germany, and, if means of transport could be found, American mills would thus be enabled to run for another year; otherwise, it was predicted that many of the mills would be forced to close in sixty days.

It was soon, however, determined to proceed to the manufacture of dye-stuffs. No Government assistance was required, but of course the circumstances in the United States were different from those prevailing here. In our case the chemical manufacturers were busy with explosives, from which they were making big profits, so that there was little inclination to take up a branch of manufacture which required much expensive plant and of which the profits were by no means so certain. At first it was stated in America that it would be exceedingly difficult to establish a coal-tar dye-stuff industry in that country, because the German industry was so dependent on interlocking chemicals ; it had reached such a state of complexity and interdependence that it would be very difficult to translate it as a whole. As regards the chemical and engineering work, no difficulty was expected, for it was stated that the production of a useful illuminating oil or a medicinal preparation from crude petroleum compared very favourably in technical manipulation with the production of most coal-tar dye-stuffs. Moreover, the petroleum products were of greater economic value. It was pointed out that to establish a complete coal-tar dyestuff industry, 700 different dye-stuffs would require to be made, and to be made properly, as good qualities of the same products would be in competition on the market. 
It was one thing to develop such an industry and to maintain it against newcomers, but another to build it up in the face of competition and to hold it against those who developed the business, knew all its ins and outs, and had their experience bought, paid for, and written off long ago. For America to be independent of Germany as regards dye-stuffs, it was urged that the intermediate products must be made on a large scale, and to do this they would require a protective duty sufficient to allow the trade to be developed. The present duty of 10 per cent was stated to be insufficient protection, because of the convention which controlled the industry in Germany and immediately reduced prices by the amount of the tax. The unfair competition of Germany could be easily checked, if the Government would incorporate in their tariff what was known as the "dumping clause," which forbids the importation into, and the selling of, in the United States of any products at a less price than they are sold in the country where they are produced. The manufacture of explosives would increase the consumption of intermediate products, for no country would care to be dependent on a foreign supply of toluol or carbolic acid. In self-defence, it was urged that some sort of a tariff should be created to protect the industry.

The experience of the Benzol Products Company, which started in a small way to manufacture aniline oil, was that, although a tariff of 10 per cent was placed on aniline oil by Congress, foreign manufacturers reduced their prices to a point where it was impossible to compete. Similarly in England the manufacture of alpha and beta naphthol and of $\mathrm{H}$ and $\mathrm{G}$ acids had been hindered because the Germans dumped down their large surplus.

There was a movement on foot in 1909 to revise the American patent laws on the lines of recent British amendments, but Germany negotiated a treaty, by which the German compulsory working clause was made inoperative on American inventions. But in America changes of the patent laws are not regarded of importance to the dyestuff industry, for there are quite enough dyes on which the patents have expired to constitute a large industry. A committee of the American Chemical Society reported 
that changes in the patent laws, such as compulsory licensing or compulsory working clauses, were wholly ineffective, do more harm than good, and should not be attempted.

From 1880 to 1883 , when there was a duty of 35 per cent ad valorem and 50 cents per Ib. specific, there were ten dye factories in the United States. In 1883 the specific duty of 50 cents was abolished, and within one year, it is said, five of these factories succumbed. Now only five were left, and these had had a great struggle to maintain themselves. It was stated that America could manufacture dyes, if it were made commercially possible; but, whenever conditions became normal again, it was thought that the manufacture of intermediate products would not be successful, because these products could be obtained very cheap from Europe.

A further opinion was that whether an American dyestuff industry arises or not depends on the consumers of the dye-stuffs. They have the materials, the technical knowledge, and the capital, and there are sufficient unpatented dyes to make nearly everything needed. Protection is necessary and will increase the prices of dye-stuffs for a time, but this increase in price will be an absolutely negligible item on the cost of dyed goods, and, when the industry is once established, protection will be no longer necessary. Consumers of dye-stuffs merely require to petition Congress that they want an American coal-tar dye industry.

The New York section of the American Chemical Society reported that alterations in the tariff are necessary to introduce the manufacture of explosives, medicinals, chemicals, and dye-stuffs in the United States. The United States produces sufficient quantities of raw products to supply a self-contained industry, and such an industry could be brought into being if there were a certainty of output in the nature of a demand from the home users. To be independent of all foreign nations at all times as regards the supply of explosives, or to protect the textile industries using chemicals and dye-stuffs and make them independent of foreign nations, the nation must bear the burden incident to such expansion. The Committee 
recommended: (1) to prevent the unfair underselling alleged to be practised by foreigners in the United States, an effective anti-dumping clause should be adopted; (2) the so-called coal-tar intermediate products, which are the basis of the coal-tar chemical industry, inclusive of explosives, medicinals, and dye-stuffs, should be taxed onehalf of whatever the finished dyes are taxed for tariff purposes, all coal-tar dyes without exception to be taxed alike, namely, 30 per cent ad valorem and $7 \frac{1}{2}$ cents per lb. specific.

At the beginning of the war the United States was in a fair position as regards intermediate products, such as aniline oil, aniline salts, beta naphthol, and para nitraniline, but the growth of this industry depended on the supply of benzol. Very soon the supplies of benzol became great, for in April 1915 Pittsburg was producing 20,000 gallons of benzene daily, the United States Steel Corporation 12,000 gallons, and other plants were being erected. The Benzol Products Company laid down a large works for the manufacture of intermediate products, and another company began the manufacture of sulphur colours. The Cambria Steel Company arranged to manufacture dye-stuffs from six tons of benzene and its homologues yielded daily by their coke plant. Other firms established plants for making dye-stuff's, and existing plants were extended. The daily output of benzol from coke works was brought up to more than fifty tons, but the manufacture of intermediates was hampered by the demands for benzol and toluol for explosives. The output of the works making intermediates is now sufficient to meet the needs of American dye-stuff makers, and other works are being erected. Two new companies have been organised for the manufacture of coal-tar products, and the other works have increased their plants for the turning out of finished dyes. There is now a steady increase in the output of American made coal-tar dyes. The large works at Buffalo are running night and day, employing 600 operatives. The volume of the output is at least double what it was before the war. Manufacture is concentrating on a few staple dyes, which are chiefly in demand, and the lack of which would be severely felt by the great textile interests. A new direct black has been 
produced, which is capable of replacing aniline black to some extent, and also some new sulphur blues. The Bayer Company's works, after being closed for some months, has now reopened and resumed the manufacture of nigrosines, alkali blue, and a few other colours. Instead of getting their intermediates from Germany as formerly, these are now made from crude American products. This firm is also making some of its pharmaceutical specialties.

The combined efforts of these works will certainly relieve the situation somewhat, but it is stated that at the best they can only replace for the next few months a relatively small quantity of the lacking colours. The firms devoted to the production of dye-wood extracts are making ample preparation for meeting heavy demands during the next few months ; increased attention is being devoted to quercitron, the only natural dye-stuff indigenous to the United States.

As the result of representations made by various interests, a Bill has been introduced into the House of Representatives with a view to the protection of the American dye-stuff industry from foreign competition. All substances produced in commercial quantities by the destructive distillation of coal, and are not themselves dye-stuff's, are to bear a duty of 5 per cent ad valorem. The duty on "intermediates" made from these substances is to be $3 \frac{3}{4}$ cents per $1 b$. and 15 per cent ad valorem, and on the finished dye-stuffs $7 \frac{1}{2}$ cents per $\mathrm{lb}$. and 30 per cent ad valorem. Coal-tar, wood-tar, acetic acid and other acids, and natural indigo are placed on the free list.

In Japan it was reported that stocks of dye-stuffs were very short at the beginning of the war, and that the situation was very exacting. Previously they had imported dye-stuffs of the value of $£ 700,000$, chiefly from Germany. As most of these dye-stuffs were used in the important textile industries, and as prices advanced rapidly, the Government introduced a Bill granting subsidies to companies making dyes and chemicals in Japan, provided that more than half the capital was subscribed by Japanese subjects. The amount of the subsidy will be sufficient to enable the companies to pay 8 per cent on their paid-up capital. Attention was also devoted to 
the cultivation of the indigo plant, a very old Japanese industry. Later, three companies were organised with a view to obtaining Government subsidies. One company, engaged in the manufacture of glycerin, with a capital of $3,000,000$ yen, intends to take over the business of an oil company at Osaka. The second company, manufacturing medicines and chemicals, intends to specialise in the manufacture of formalin and its derivatives, and in other carbon compounds. This company will have a capital of 500,000 yen, and will acquire the works of an existing Japanese concern engaged in the production of acetic acid. The third company produces medicines, and will have a capital of 1,000,000 yen. The Japanese Government has arranged to form a company for the manufacture of dyestuffs, with a capital of $8,000,000$ yen. The first works will be built in Tokio or Osaka, or in the north of Kuishin (probably close to the Wakamatzu ironworks, whence the necessary benzol will be obtained).

In Russia a syndicate was formed, composed of some of the largest industrial concerns, for the establishment of works for making dye-stuffs and for the erection of special research laboratories. The assistance of a number of technical chemists from Switzerland was obtained.

One of the reasons attributed for the failure of the French to establish a coal-tar colour industry is the patent legislation, according to which a new substance may be made the subject of a patent, so that the inventor of a much preferable method of production is unable to work the new process until the patent for the substance itself has expired. Many examples of such hindrance are known. For instance, the indanthrenes, an important class of dye-stuffs, were discovered and patented in France, but were eventually marketed by German firms because the original process of production was less economical. Prohibitive fiscal measures will not of themselves, it is said, assure the building up of a coal-tar industry, for success in this direction depends more on educational and commercial considerations. Then there is the difficulty of distinguishing for fiscal purposes between raw materials, intermediate products, and finished products. For example, the colourless leuco bases of triphenylmethane 
dye-stuffs have been admitted to France as intermediate products.

The manufacture of dye-stuffs is now established at Paris, Nancy, and Lyons, and extensions of the works have been decided upon, but at present there is a shortage of crude tar products, which previously were imported from Belgium, Great Britain, and Germany. It is not regarded as probable that sentiment will play much part eventually in the purchase of dye-stuffs; hence it will be essential to offer colours at practically the same prices as German firms. The manufacture of colours, for which the patents have expired, can be taken up, but this business is not very remunerative, because such colours are generally low in price since the patentees have made their profit out of them and further developed their plant and process. The attraction of chemists trained in the production of dye-stuffs was a difficulty, but it was thought that some assistance might be obtained from Switzerland such as Russia had obtained.

It is the author's opinion that the enterprise of British Dyes, Ltd., will be a great success. Moreover, the value of the German dye-stuff industry will decrease as the industries of England and America, hitherto Germany's best customers, increase. The establishment of the aniline-colour industry in this country will also give the public a concrete example of the value of science to industry, and will prevent politicians from pointing to the decadence of England as instanced by the anilinecolour industry. By providing employment for trained chemists it will also react in helping the universities and technical education in general.

There seems to be a consensus of opinion that after the war prohibition for a time of German imports, and then a high tariff, will be the only way of protecting the industry. While in Germany the author was often told that a tariff put on German manufactures by England would have meant war between the two countries, but such a consideration is not now of interest. Certain it is that, from what has been stated above, some protection will have to be provided for the new enterprise. Free Traders have always admitted that protection is sometimes 
of advantage in developing new industries; in this case international trade will have to be arranged with the military situation in view. But it might not be advantageous to our textile industries to exclude all German dye-stufts after the war, because this procedure might affect us in competing in foreign markets. For instance, such colours as Bayer's alizarine brands may be found difficult to make, and if kept out of this country may be a handicap to our textile industries. A great deal will depend, however, on the progress made by British Dyes, Ltd.

Mr. Runciman, the President of the Board of Trade, in the House of Commons on January 10, said: "Of course we could not go on with our trade treaties in the same way after the war. Some of them did the Empire grave injustice. The working of the most-favourednation clause had operated increasingly to our detriment in recent years under the arrangements made between Germany and Russia. We could not permit at the conclusion of the war the outbreak of another economic war by Germany against us. Our own national and commercial interests must in all these matters be our first and only guide. Some industries were almost entirely in the hands of Germany before the war. We were now producing optical glass and a large number of chemical articles, which previously were almost entirely in German hands. The same thing applied to dyes, electrical apparatus, and other articles. We had been placed at a great disadvantage owing to the lack of these articles in this country. Never again should that happen. There should be no essential article, either for the arts of peace or war, which we could not in the Empire lay our hands on. Progress in this matter did not depend on Government assistance. Government departments could do a great deal, but without the personal ability, training, skill, and knowledge of the individual nothing could be done. The first interest of this country was to improve the training of our own men, and we should not attempt to economise in this direction. The next quality was that of adaptation. The war had taught the commercial people of this country the necessity of adaptability." $\mathrm{He}$ 
agreed that there should be an extension of commercial banking as distinct from the more conservative form of banking business. Manufacturers and commercial men were at a disadvantage as compared with those in Germany who were in touch with more adventurous institutions. Our banks must be more enterprising. If they could not, then we must have some additional institutions.

$\mathrm{He}$ also agreed that we must overhaul our patent system. However much we might help industry by changes of policy, there was no doubt that the development of new inventions, and restrictions put on their use, would have a greater influence on the extension of our trade and industry than even the juggling of a tariff.

Of the 700 or more colours made by the German works, only a small proportion need be made in this country in order to supply our wants during the war or, indeed, after the war. It is important to make large quantities of a few colours. Many of the German colours gained a market merely because of their cheapness, but price is not a vital consideration at present. Large quantities of the better colours are necessary. Research work will be principally required at first to ascertain methods of production of known colours, in order to prepare these products on the large scale, but afterwards work can be done in the way of discovering new compounds.

It has been stated that after the war large quantities of dye-stuffs will be made in America under German control, and that these dye-stuffs will be exported to this country; but it must be remembered that America will have to strive very hard to supply her own needs. If, however, she is able to export some dye-stuffs, our industry can be protected from these imports in the same way as it will be protected from dye-stuffs coming directly from Germany. There is no doubt that great efforts will be made in America to retain the industry which they are building up in war time; if an export trade follows, there are plenty of other markets to supply besides ours. We ourselves, in the meantime, had better give our whole attention to our own requirements.

In considering the aniline-colour industry it must always be remembered that through chemical research is 
the road to success. The German and Swiss works, which have brought out the greatest inventions, are the principal works devoted to the industry, and their success has been due to the research work which has resulted in these inventions. The only way that our new industry can flourish is by developing in the same direction, that is, by employing research chemists from our universities. In the German aniline-colour works there are hundreds of research chemists trying the effects of introducing different groups into the molecules of known dye-stuffs, and by a variety of other more or less stereotyped ways preparing new organic compounds. New dye-stuffs produced are submitted to technical men for trial; some are found worthy of manufacture and others are rejected. And even if adopted they might be little used in industry. Now, if a large number of men are continually engaged in this work, it is obvious that some discoveries must result, although it must be admitted that there is an element of chance in the whole business. Moreover, when one considers that many of the best products of these works have been discovered outside the works laboratories, it is manifest that each one of the research chemists in the works cannot have achieved many distinct triumphs.

If our new colour industry engages a number of research chemists, an outlet for our organic chemists will thus be provided. The author has long maintained that it is only by such means that organic chemistry can flourish. In Germany the colour works employ large numbers of chemists trained in organic research, and in this way encourage the study of this branch of science. We must make organic chemistry a "bread-and-butter" study. If the new colour industry offers students, who have done research work in organic chemistry, a position at, say, $£ 100$ a year and a share in all remunerative discoveries, it will not be long before there is a large supply of organic research chemists. When students have finished their three years' honours course in chemistry at an English university, and have perhaps done a little research work, they look out for a remunerative appointment. In some cases a research scholarship is obtained, but the competition for these scholarships is keen, and it would be 
much keener if the graduates could see some definite position ahead after the scholarship is finished. After the research work has been completed and published, these scholarships generally lead to minor staff appointments, and in some few cases to professorships. But the graduates who do not succeed in obtaining research scholarships have to find other outlets for their abilities, and the tendency has been in recent years for honours chemistry graduates to obtain positions as science masters at grammar schools, that is, to find refuge in positions which are not designed for them, and are more suitable for men who have taken the ordinary science degree. When these situations are obtained, the honours man certainly receives a living wage at the outset, but he finds that his opportunities of further advancement are few, for he has little chance of doing research work or of distinguishing himself in other ways. In short, his honours training has not been utilised as it should have been, and has had no opportunity of developing its true usefulness. This position has really arisen because of the few opportunities which are presented to the honours graduate of obtaining a living; for it must be remembered that there are comparatively few students in the chemical laboratories of our universities who can afford to study the science without giving consideration to the position to which the study will lead. If an outlet for these honours graduates can be found in the new industry, there will be a great impetus to the study of organic chemistry and a direct gain to the industry. In fact, by providing such positions is the only way in which the new aniline-colour enterprise can achieve success, and such an action will make it absolutely unnecessary for the Government to make lavish grants in aid of so-called research. Each position so granted will yield its own reward, and it is only by a commercial transaction of this nature that research and university chemical training can receive real assistance. These remarks also apply to other branches of chemical industry. The primary consideration is - get trained chemists into industry.

The study of chemistry in this country has hitherto opened the door to few employments, hence it has shown 
a tendency towards becoming a kind of luxury dabbled in by those who liked it and could afford to pursue it regardless of profit. Under such circumstances the study could not flourish. Professor Perkin has lately mentioned his father's natural dislike for an industrial career and his desire to devote himself entirely to pure chemistry. That resolve proved to be of advantage to pure chemistry, but it was certainly unfortunate for our infant colour industry. It is still more unfortunate, however, that so many chemists in this country are ready to follow in Sir William Perkin's footsteps, for among scientific chemists connection with industry is not liked, and industrial work is looked upon as being of "merely technical" interest. Work known to have taken many years of hard labour, work scientifically carried out and sometimes of high importance to the community, has been overlooked because it was said it made no direct contribution to science. The result is that research students aim more and more at abstract research, and attempt to introduce higher mathematics into their work so as to squeeze papers into the transactions of learned societies, and thus receive recognition. They know at the outset that technical work will not advance them in chemical circles. This state of affairs cannot be of use in assisting the application of science to industry.

Another state of affairs which must be mentioned, and also deplored, is the feeling of distrust which exists between what are called scientific chemists and technical chemists. Although many scientific chemists refuse to have any connection with industry, this feeling does not prevent them from lecturing industrial chemists from time to time in an attempt to give them advice concerning industries of which they themselves have only an outside knowledge. These attempts, however, only lead to irritation. It must be remembered that there are many really distinguished chemists engaged in the industry of this country who have no university degrees and to whom contributions to the transactions of learned societies offer no attractions. It is in the interests of the co-operation of science and industry that a spirit of esteem should be engendered between the two classes of chemists mentioned. 
As for the contention that in Germany the professors of chemistry are far more in touch with industry than they are in this country, the author found little evidence in favour of this view. For instance, he met Professor F. Haber at Karlsruhe, a chemist who has since become distinguished. Professor Haber was engaged on a number of researches at the time, and he gave the author a number of introductions to works in the district, but it was evident that at that time this professor was not in intimate association with industry. Shortly after the author's visit, however, Haber was brought into association with industry, but this event was a direct result of his discoveries. Such work would have resulted in industrial connection in almost any country. Again, take the discovery of salvarsan by a German professor, and its manufacture at the Hoechst Works. Can it be maintained that, if such a product had been discovered at an English university, a chemical works could not have been found in this country to take up its manufacture? In such cases as these, industrial connection was bound to arise : the merit of the discoveries brought about the connection. But it is only in such cases that we find a close connection between professors and industry in Germany. In England, however, we find professors openly stating that they have never done consulting work or had any connection with industry while holding their professorship, and this they consider a right position to take up. A chair at a university might be founded from money made in industry, but the professor holding the chair might refuse to have any connection with industry. There is no point in our scientists calling for co-operation between science and industry if they themselves do little to encourage the connection; for success in this matter can only be achieved by the urgent desire of both parties interested. 



\section{INDEX}

A

Additions to scouring lyes for bleaching, 41

Advantages of coal-tar colour industry to Britain, 129

to Germany, 84, 97, 117 of education not always seen, 84

Algol colours, 19, 115

Alizarine colours, 2, 130; blue, 18; cyanine, 18 ; production, 98

American and English workers compared, 60,72 ; conditions, 49 ; dyeing industry, 49 ; employers and education, 81; hank-mercerising machine, 23 ; home trade, 49 ; machinery, 49 ; mercerising machines, 26,27 ; trusts, 99

- workmen, 72

Analytical chemists at German colour works, 107, 108

Application of chemistry to bleaching, 32 of science to industry, 134

Appointments for chemists in British industry, 132

132,133

for technical students, 83

Arlington Mills, Lawrence, 61, 73

Arnold Print Works, North Adams, 55, 73

Arrangement of operations in bleachworks, 59

Art of dyeing, 1

Assistance afforded to manufacture, 74

to chemical training, 133

Austria, technical instruction in, 86

Automatic counting machines, 60

\section{B}

Badische Anilin und Soda Fabrik, 15, $67,97,98,103,113$; technical laboratories, 113

Banking reform in Britain, 131

Barmen, 3, 5, 8, 66, 75

Barnaby Works, Fall River, 63

Basic blues, 17
Bayer Co. of Elberfeld and Leverkusen, $18,19,97,103,106,127,130$; development of, 103 ; employment of chemists, 104 ; patent specifications, 104 ; water-supply at Leverkusen, 106

Beetling, 57

Bemberg's dyeing and machine works, Barmen, 35, 75, 76 ; hank-mercerising machine, 21, 22 ; piece-mercerising plant, 25

Benzol Products Co., 124, 126

Berlin Aniline Company, 75, 97, 104, 113 ; technical laboratories, 113

Black smoke in U.S.A., 75

Bleaching, agents, 37; application of chemistry to, 32 ; by electrolysis, 37 ; empiricism in, 32 ; history of, 31 ; industrial, 32; without complete scouring, 41

Bleachworks' organisation, 59

Blues, alizarine, 18; basic, 17 ; benzo, 17; developed, 17; diamine, 17; direct, 17 ; sulphur, $12,15,18,19$, 61 ; vat, 18

Books on technical instruction, 81

British Alizarin Co., 98

British chemical industry, effect of war on, 130 ; colour industry and German patents, 122 ; colour industry during war, 122 ; Dyes, Ltd., 129 ; efforts to found colour industry, 120; Empire and industry, 130; Government attitude to colour industry, 119

Business management of German colour works, 101, 104

\section{C}

Cachou de Laval, 12

Canals, use in industry, 74

Cassella, L., of Frankfort and Mainkur, $12,15,18,97,112$; experimental laboratories, 112

Cell dryer for cloth drying, 62, 76

Cellulose, 32

Changes in dyeing industry, 80

Charlottenburg technical high school, 86 


\section{DYEING IN GERMANY AND AMERICA}

Cheap chemicals and colour industry, 95 Chemical graduates and appointments, 132 ; research and colour industry, 131

Chemistry and bleaching industry, 33 ; and dyeing, 2 ; professors and consulting work, 85, 135 ; Professors of, and industry, 135 ; tuition in dyeing schools, 85

Chemists and abstract research, 134 ; and industry, 92, 133, 134 ; in British industry, 134; in German colour works, 72 ; in German industry, 71

Chemnitz, 3, 4, 5, 9, 56, 66, 71, 75, 85 ; Staat-Lehranstalt, 86

China, natural indigo in, 17

Chloramines, 41

Ciba colours, 19, 115

Circulation during bleaching, 63

Cleanliness in industry, 65

Cloth-dyeing, 5

Coal-tar colour works of the world, 118

Cocheco Manufacturing Co., Dover, N.H., 76

Cochineal, 1

Cohnen cop-dyeing machine, 7 ; hankmercerising machine, 23

Colour industry in France, 129

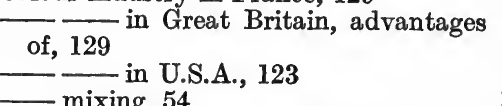

- works, influence on education, 84

Columbia University, New York, 90

Combination in German colour industry, $97,98,100$; of employers on labour questions, 57

Commercial adaptability, 131

Comparison of manufacturing conditions, 67

Competition and prices of dye-stuffs, 98 ; in colour industry, 97, 100, 114

Compilation of patent specifications, 103

Compulsory working of patents, 102 , $115,124,125$

Conditions in old and new works, 66

Considerations to industry, 74

Consumers of dye-stuffs and colour industry, 121, 125

Contracts with servants in German colour-works, 109

Co-operation of science and industry, 134, 135 ; between works in Germany, 117

Cop-dyeing, 6 ; by frothing, 113 ; dyestuffs for, 6 ; faults in, 8 ; origin of, 6

machines for, 7 ; with perforated spindles, 9 ; with solid spindles, 8

Cotton blankets, 57

Cotton speculation, effect on industry, 56

Counter currents, principle of, 5, 23
Crefeld, 3, 27, 71, 75, 85 ; Fachschule, $85,86,87$

Crimping of cloth, 20

\section{D}

Decline of colour industry in England, 94

- in France, 128

Degrees in Germany, 83

Dependence of England on German dye-stuffs, 99

- of Japan, 127

- of U.S.A., 123

Development of the art of dyeing, 1

Direct blues, 17 ; cotton colours, 17

Distribution of the dyeing industry, 3

Drying cylinders, arrangement of, 62

Drying of dye-stuffs, 105

Dumping of German dye-stuffs, 98, 124, 126 ; legislation against, in U.S.A., 126

Dutch flannelettes, 48

Duty on dyed cloth, 2

Dyeing industry in U.S.A., 72 ; information collected at German colourworks, 91

Dyeing machine, special, 112

schools supplying own needs, 88

Dye-stuffs, drying of, 105 ; manufacture of, 105

Dye-works and machine-shops together, 75,76

\section{E}

Ecob and Hasslacher mercerising machine, 25

Economic relations with Germany, 130

Economy of high wages, 55, 69

Edmeston continuous kier, 36

Education in Germany, 69 ; of classes in Germany, 83 ; of poor children, 69

Educational ladder in England, 83

Effect of colour industry on other chemical industries, 95

- of lock-out in Lowell, 57

- of tariffs in America, 51

- of war on dye-stuff supply, 115, 118 in France, 128 in Japan, 127 in Russia, 128 in U.S.A., 123

Efficiency in English dyeing industry, 78

Efforts of U.S.A. in colour production, 126,131

Eight-hours day in industry, 70

Elberfeld, 3, 66

Electrolysers, 38

Electrolytic bleaching, 37, 38, 39

Eminence of English dyeing, 78

Empirical methods in industry, 73, 80 
Employees' advantages in German colour-works, 107

Employers and education, 88, 90, 92, 93

Employment in American print-works, 64

- of chemists in dye-works, 64

Encouragement of labour, 58; of research in Germany, 96

England and chemical industry, 81

English and Continental technical schools, 86

English chemists in Germany, 84

English coal-tar dye-stuff industry, 94

English colour firms, 101

cotton-waste used in Germany, 44

employers and education, 81

foremen and managers in U.S.A., 60,72

- graduates and industry, 84

industrial position, 82

99

industry and German dye-stuffs,

- instructors in American schools, 89

machinery in Germany, 3, 44, 85

pessimism regarding education, 81 regarding industry, 82

students in German industry, 88

Englishmen in American industry, 60, 72

Engraving of printing-rollers, 58

Entrance examinations at American technical schools, 90

- qualifications at German technical schools, 87

Evasion of patent laws, 102

Examinations in Germany, 83

Excellence of British dyeing, 90

- technical schools, 90, 110

Experimental dye-houses at German colour-works, 112

Expiration of German colour patents, 103,124

Explosives and dye-stuffs, 124, 126

Export trade in American prints, 51

External specialisation in Germany, 47 ; in U.S.A., 50, 77

Extremes of remuneration, 69

F

Factory Regulations, 65

Faculties of commerce at universities, 82

Fall River, Mass., 3, 50, 61, 88; specialisation at, 61 ; water-supply, 62

Fall River Bleachery, 62; Ironworks, 62 ; Textile School, 88, 89

Fastness of dye-stuffs, 113

Fees in German technical schools, 85, 86

Felting, brushing, and steaming of flannelettes, $44^{\circ}$

Fine and coarse goods at Lowell, 56

Fine chemicals in Great Britain, 116, 117
Finishing, 51

Foam dyeing-machine for cops, 11, 113

Foreigners in German technical schools, 85

Foundation of colour industry in U.S.A., 123

France and chemical industry, 81 ; the colour industry, 128

Free tuition in Austrian technical schools, 86

Future of English colour industry, 94, 129

- of German colour industry, 96, 129

G

Gebauer kier, 34; machine-works, Berlin, 75,76 ; open-width kier, 35

Gera Mills, Passaic, N.J., 50, 51, 76

German chemists and English industry, 95

— colour firms in U.S.A., 97

—— industry a monopoly, 98 patents, 101

dustry, 122

- works, 91, 94

101

- educational institutions, 81

- exports of dye-stuffs, 118, 119

graduates and industry, 84

- grants to technical education, 84

— kartels and home price, 99

— patent specifications, 115

— professors and industry, 135

— railways and industry, 105

- technical schools, 84

Government facilities to industry in Germany, 117

Grämigers cop-dyeing machine, 9

Gruschwitz piece-mercerising machine, 27

\section{$\mathrm{H}$}

Hamilton Works, Lowell, 59

Hank-dyeing, 3, 4; in U.S.A., 4, 60 ; Klauder-Weldon machine for, 4, 14, 63 ; machines, 4

- mercerising, 21 ; machines, 21, 23

- washing, 4, 5 ; machine, 4

Haubold's cop-dyeing machine, 9 ; hank-mercerising machine, 21, 22 ; hank-washing machine, 4 ; kier, 34 ; machine works, Chemnitz, 75, 76; special dyeing machine, 112

Heavily filled cotton goods, 51

Heavy chemical industry, 116, 117

History of bleaching, 31

of dyeing, 1

Holland, 11, 31, 118 ; colour industry in, 118

Home and export trade in flannelettes, 45 
Home demand and colour industry, 125

Honours chemistry graduates in England, 132

Hours of work in Germany, 69

- in U.S.A., $55,58,60$

Housing of working-classes, 107

Hydrogen peroxide as a bleaching agent, 37

\section{I}

Ice-making in German colour-works, 106 Immigration, effect of, on American labour, 49

Importance of chemistry in technical education, 85

of colour industry to Britain, 96

Indanthrene colours, 19

Indigo, 1, 15, 63

dyeing, 112

fading of, 19

natural, 15; cultivation of, 16 ;

in China, 17; in Japan, 17; revival due to war, 16

- substitutes, 17

synthetic, 15, 118; and natural compared, 16 ; effect of war on, 16

Industrial combination in England, 99

- in Germany, 99

—— in U.S.A., 99

- research in Germany, 135

Inflammability of flannelettes, 48

Influence of colour-works on education in Germany, 84, 96

Information collected by German colour-works, 91

Injurious conditions in dye-works, 65

Inorganic chemistry in England and France, 83

Intermediate products, manufacture of, 125,127

Italian flannelettes, 48$$
\text { J }
$$

Jackson-Hunt kier, 34, 36

Japan, colour production in, 127; natural indigo in, 17

Jeanmaire's piece-mercerising machine, 24,25

Jiggers for dyeing, $5,57,59$

K

Kalle \& Co., 19

Karlsruhe, 86 ; Technical High School, 86,135

Kartels, 99

Kiers for scouring, 33; circulation in, 33 ; low pressure, 62

Klauder-Weldon hank-dyeing machine, $4,14,63,89$; raw-stock dyeing machine, 61

Kleinewefer's, Crefeld, 21, 75, 76 ; hankmercerising machine, 21
L

Labour-saving machinery, 59

scarcity in America, 49, 55

Land at reduced rates for industry, 75

Large-scale production, advantages of, $45,75,99$

- in U.S.A., 50, 63

Lawrence, Mass., 50, 55, 60

Leather dyeing, 113

Lectures in economics, 91

in patent law, 85, 91, 102

Libraries at German colour-works, 109

Lime-boil in bleaching, 40

Location of dyeing industry, 3

Logwood, 2

Loose cotton mercerising, 21

Low-pressure kiers for bleaching, 62

Low wages and long hours in Germany, 69

Lowe, 20

Lowell, Mass., 53, 56, 73, 75, 88 ; Textile School, 73, 88, 89

\section{M}

Machine-building at dye-works, 56 ; industries in new countries, 56

Machinery used for flannelettes, 46, 47

Machines for raw-cotton dyeing, 61

Manchester machinery, 44; printers, 45

Manufacture of a few necessary colours, 126,131

of dye-stuffs, 105

Manufacturing conditions in Germany, 66

Massachusetts Institute of Technology, Boston, 61, 89

Meister, Lucius \& Brüning, Hoechst, $12,97,98,107,135$; experimental laboratories, 112

Mercer, 20

Mercerising, 20; additions to lye in, 21, 28 ; economy of soda in, 26 ; imperfect, 26 ; prices for, 27 ; research in, 79; strength of soda for, 20 ; temperature of soda for, 20 ; tests for, 29 ; trade in Crefeld, 26

Merrimac River, 60

Merrimack Manufacturing Co., Lowell, $26,27,53,73$

Metallic colours, 106 mordants, 2

Milling machine, 113

Modern inventions, 72

Mommer cop-dyeing machine, 7, 11

engineering works, 76

Monopoly in German colour industry, 98,100

Most-favoured-nation clause, 130

Mülhausen, 3, 5, 36, 66, 88, 107; Dyeing School, 85 
München-Gladbach, 3, 43, 47, 84, 88, 98 ; Textile School, 84, 87

Muntadas (Paris) continuous kier, 36

\section{$\mathrm{N}$}

Nationalities engaged in American industry, 49, 60

New Bedford, 50,89

New England, 3, 49, 50, 51

New methods in industry, 78

New technical literature, 78

New York, 3, 90, 125

Non-inflammable flannelettes, 48

North Adams, Mass., 54, 55, 73

Numbers of students at German technical schools, 86,87

Nüremburg, 106; lithographic colours at, 106

\section{0}

Obermaier cop-dyeing machine, 8, 11

Open-width scouring, 34

Opinions regarding technical education in U.S.A., 88,89

Opposition to artificial dye-stuffs, 97

to British Government dye scheme, 121

- to innovations, 2,97

- to introduction of new machinery, 58

- to progress, 2

Organic chemistry and British colour industry, 132

97

- and German colour industry,

- in Germany, 84

Organisation of English flannelette trade, 46

of German flannelette trade, 46

of industry in U.S.A., 50

\section{$\mathbf{P}$}

Pacific Mills, Lawrence, 61

Padding, 14, 57, 59

Paper spindles used in cop-dyeing, 8

Passaic, New Jersey, 49, 50, 68, 75

Patent legislation, 131; in England, 102 ; lectures on, 85, 91

Patented products of German colour works, 102, 115

Patents and the colour industry, 102, 115, 124, 129

Pattern books supplied by German colour firms, 104

Payment of royalties on British dyestuffs, 122

Pemberton Mills, Lawrence, 60

Pharmaceutical chemicals, 116

Philadelphia, 3, 89

Photographical chemicals, 116

Piece-mercerising machines, 21, 24
Polygenetic colour dyeing, 1

Pornitz cop-dyeing machine, 9, 10, 113

Position of German colour industry, 96

Post-graduate research, 132

Prejudice against technical education in U.S.A., 88, 89

Prices for dyeing, 71

- of dye-stuffs, $71,91,98,131$

- of materials used in dyeing, 91

Printing, 14, 50, 53, 54

Production of chemicals in Great Britain, 116

Production of new dye-stuffs, 115

Profits from dye-stuffs, 96, 98

Prohibition of importation of German colours, 129

Protection and the colour industry, 101, 125

- and the American colour industry, 125,127 129 and the British colour industry,

Publication of research in journals, 78

$\mathrm{R}$

Railway development in U.S.A., 74

Raising of cotton fabrics, 43

Ratepayers and education, 84

Raw cotton, constituents of, 40 ; dyeing, 60,61

Raw materials and taxation, 101

— for German colour industry, 95

Read, Holliday \& Sons, Ltd., 119, 122

Recovery of waste products, 74

Reichenberg Weaving School, 86

Report of Moseley Commission, 81

Reports of commissions on education, 81

Research and British colour industry, 132

chemists and the colour industry, $95,107,108,111$

— in German colour industry, 111

- remuneration of, 111

- encouragement of, in Germany, 96

—_ in dyeing in technical schools, 88

- in dye-stuff preparation, 111

in English universities, 132

- in German technical schools, 85, 86

in pure chemistry, 134

on mercerising, 79

scholarships in chemistry, 132

work in German colour industry, $103,131,132$

- in industry, 78

Resources of U.S.A., 72

Restrictions on dyeing industry, 1

Rhine and German commerce, 104

Rights obtained by examination in Germany, 83 
Royalties and British colour industry, 122

Russia and the colour industry, 118, 128, 129

\section{$\mathrm{S}$}

Salts Manufacturing Co., Bridgeport, Conn., 51, 63

Saxony, 3

Scarcity of labour in U.S.A., 49, 55

Schirp cop-dyeing machine, $8,9,10$

Schreiner calendar, 76

Schwabe's piece-mercerising machine, 24

Science and empiricism in bleaching, 32 and the direction of colour-works, 121,122

Scientific and technical chemists, 134

- management, 55

- skill in industry, 71

Scouring, 41 ; effect of neutral salts in, 40

Secrets in industry, 54, 72

Self-made men in English industry, 69, 92

Sentiment and purchase of dye-stuffs, 129

Singeing machines, 62

Skin dyeing, 113

Small colour-works in Germany and Switzerland, 101

Society of Chemical Industry, Basle, 18 Sodium hypochlorite in bleaching, 37

_ _ produced by electrolysis, 37 peroxide as a bleaching agent, 37

Sorau technical school, 87

Southern states of U.S.A., 3

Spanish flannelettes, 48

Special lectures at technical schools, 89

students at technical schools, 88

Specialisation at Fall River, 61

in printing, 55

in textile machine industry, 56

- internal and external, $46,47,55,76$

Specialised textile machine-shops in

England, 76

Speed of machines, 58

- in bleachworks, 63

Spinning the weft for cotton blankets, 44

Stentering machines, 57

Strength of dye-stuffs, testing, 74, 113

Strikes in Germany and U.S.A., 67, 68

Students at German technical schools, 87 ; entering industry, 92

Study governed by outlets for students, 82; of chemistry in England, 133; of English in Germany, 83; pursued for professional object, 83

Success and research in colour industry, 131

due to employing new machines,
Success due to paying high wages, 55 to scientific management, 55 of German colour industry, 95

Successful methods in German colour industry, 103

Suggested improvements in technical instruction, 91

Sulphur blacks, 12, 15

- blues, 12, 15, 18, 61 ; fading of, 19 violets, 12

Sulphur colours, 12 ; machine used for, 14, 15; methods of dyeing, 13; padding of, 14 ; printing with, 14 ; research in, 111 ; tendering due to, 12 Synthetic and natural indigo, 16

Synthetic drugs, 116

Swiss colour firms, 18, 101, 118, 128, 129, 132 ; competition with Germans, 97

\section{$\mathrm{T}$}

Tagliani-Rigamonti kier, 35, 36

Tariffs and dye-stuffs in France, 128 in U.S.A., 124, 125, 126, 127

- and German exports, 129 and the colour industry, 101

Technical chemistry at American schools, 89

- departments of German colourworks, 107, 109

- education, 81, 83 ; in Austria, 86 ; in Germany, 81 ; in U.S.A., 88 ; of workmen in Germany, 87

- instruction in dyeing, 80

— men and British colour industry, 110 ; in German colour-works, 100, 107,108

- periodicals, 72,78

- research work, 134

- schools and industry, 92 ; schools as leaders of development, 91

- training and the dyeing industry, 80 ; and works experience, 93 ; at German colour-works, 109, 110; in England, 130

Tendering due to imperfect scouring, 41

Testing of dye-works supplies, 73

Textile Institute, the, 91

Textile machine-shops attached to dyeworks, 75

Theis's cop-bleaching apparatus, 11

Thio indigo red, 19, 115

Thomas \& Prevost, 20

Trade in coal-tar dye-stuffs, 118

Trade unions, 58; in Germany and U.S.A., 67

Training of managers in American technical schools, 88

Transport facilities to German industry, 104

Travellers employed by German colourworks, 108 
Turkey red and the American market, 57

\section{U}

Uniformity of nap on German flannelettes, 45

University graduates and British colour industry, 132

\section{9}

at German colour-works, 108,

students and textile schools, 87,90

Use of canals, 74

V

Variety of products at German colourworks, 111

Vat blues, 18; dye-stuffs, 19

Ventilation of dye-works, 66

Vidal black, 12

Vienna weaving school, 86

Visiting of works in U.S.A., 53

- by other workers, 53

—— by technical students, 90

Volatile solvents used in scouring, 41

Volunteer departments in German colour-works, 87, 112, 114
W

Wages in Germany, 69; in the dyeing industry, 68,71 ; of chemists, 71

Walsh-Jackson kier, 34

War and the chemical industry, 130

Warp mercerising, 21, 23

Water-supply at Fall River, 62; at Leverkusen, 106

Waxes of textile fibres, 40 ; effect of lime-boil on, 40; effect of hypochlorites on, 41

Weighing of dye-stuffs, 73, 113

Weisbach piece-mercerising machine, 27

Welter continuous kier, 36

Woad, 1

Wood colours, 115, 118, 127

Works experience for technical men, 110

Works training deferred, 93

\section{Y}

Yarn dyeing, 3

in U.S.A., 4, 60, 63

Z

Zwickau, 3 


\section{THE GARTSIDE REPORTS}

The Gartside Reports are the reports by the Gartside Scholars at the University of Manchester. The Gartside Scholarships were established in 1902 by John Henry Gartside, Esq., of Manchester, and are tenable for two years, and in future one will be awarded each year. They are open to males of British nationality who at the date of election shall be over the age of eighteen years and under the age of twenty-three years.

Every scholar must enter the University of Manchester for one session for a course of study approved by the electors. The remainder of the time covered by the Scholarship must be spent abroad and devoted to the examination of some subject bearing upon Commerce or Industry. It is intended that each scholar shall select some industry or business for examination, and investigate it comparatively in the United Kingdom and abroad. The first year's work at the University of Manchester is designed to prepare the student for this investigation. Finally, each scholar must present a report, which will, as a rule, be published.

The value of a Scholarship is about $£ 80$ a year for the time spent in England, $£ 150$ a year for time spent on the continent of Europe, and about $£ 250$ a year for time spent in America. 



\section{UNIVERSITY OF CALIFORNIA LIBRARY BERKELEY}

\section{THIS BOOK IS DUE ON THE LAST DATE \\ STAMPED BELOW}

$50 \mathrm{c}$ per volum not returned on time are subject to a fine to $\$ 1.00$ per volumer the third day overdue, increas of demand may belume after the sixth day. Beoreasing expiration of loan period.

- MAN 261918

FFR 11. 1919

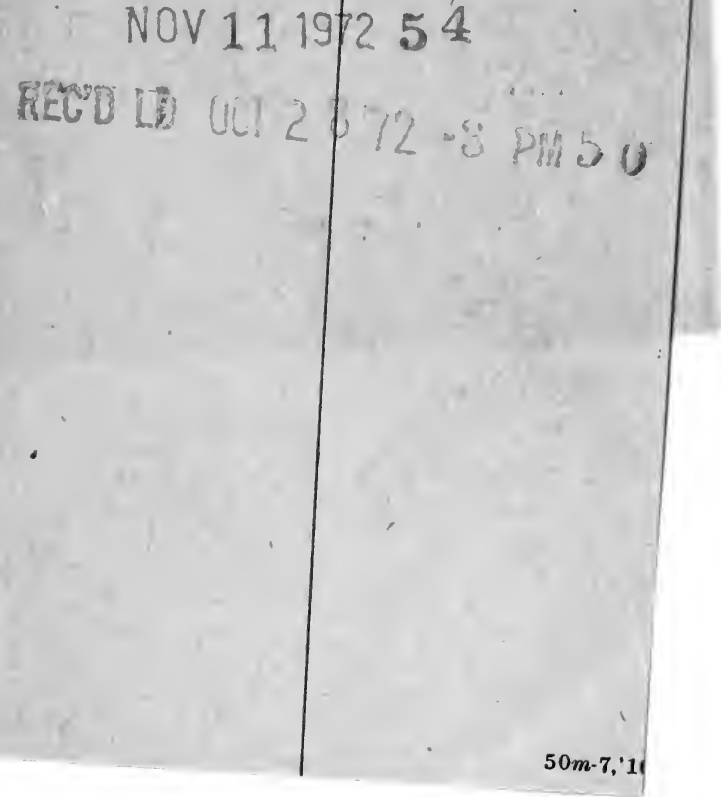


$\frac{\text { ule }}{\text { p.te un }}$

IL 81290

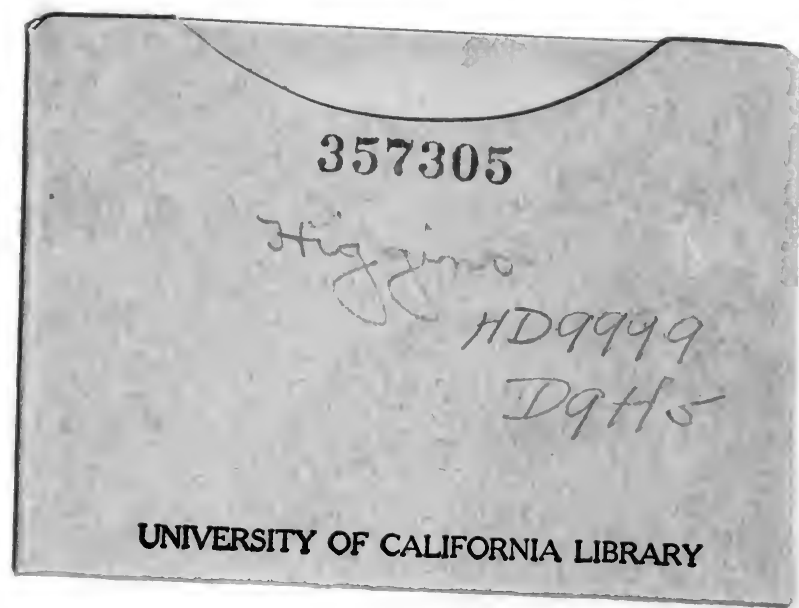


